Oct $1, \pi \rightarrow$

\title{
OSTI
}

\section{Computer Modeling of \\ ORNL Storage Tank Sludge Mobilization and Mixing}

\author{
G. Terrones \\ L. L. Eyler
}

September 1993

Prepared for the U.S. Department of Energy under Contract DE-AC06-76RLO 1830

Pacific Northwest Laboratory Operated for the U.S. Department of Energy by Battelle Memorial Institute 


\title{
DISCLAIMER
}

This report was prepared as an account of work sponsored by an agency of the United States Government. Neither the United States Government nor any agency thereof, nor Battelle Memorial Institute, nor any of their employees, makes any warranty, expressed or implied, or assumes any legal liability or responsibility for the accuracy, completeness, or usefulness of any information, apparatus, product, or process disclosed, or represents that its use would not infringe privately owned rights. Reference herein to any specific commercial product, process, or service by trade name, trademark, manufacturer, or otherwise does not necessarily constitute or imply its endorsement, recommendation, or favoring by the United States Government or any agency thereof, or Battelle Memorial Institute. The views and opinions of authors expressed herein do not necessarily state or reflect those of the United States Government or any agency thereof.

\author{
PACIFIC NORTHWEST LABORATORY \\ operated by \\ BATTELLE MEMORIAL INSTITUTE \\ for the \\ UNITED STATES DEPARTMENT OF ENERGY \\ under Contract DE-ACO6.76RLO 1830
}

Printed in the United States of America

Available to DOE and DOE contractors from the

Office of Scientific and Technical Information, P.O. Box 62, Oak Ridge, IN 37831;

prices available from (615) 576-8401. FTS 626-8401.

Available to the public from the National Technical Information Service,

U.S. Department of Commerce, 5285 Port Royal Rd., Springfield, VA 22161. 
PNL-8855

UC-510

.6

$\mathrm{OCT}$

$\mathrm{O}=$

Computer Modeling of ORNL

Storage Tank Sludge

Mobilization and Mixing

G. Terrones

L. L. Eyler

September 1993

Prepared for

the U.S. Department of Energy

under Contract DE-AC06-76RLO 1830

Pacific Northwest Laboratory

Richland, Washington 99352 


\section{Summary}

This report presents and analyzes the results of the computer modeling of mixing and mobilization of sludge in horizontal, cylindrical storage tanks using submerged liquid jets. The computer modeling uses the TEMPEST computational fluid dynarnics computer program. The horizontal, cylindrical storage tank configuration is similar to the Melton Valley Storage Tanks (MVST) at Oak Ridge National Laboratory (ORNL). The MVST tank contents exhibit non-homogeneous, non-Newtonian rheology characteristics. The eventual goals of the simulations are to determine under what conditions sludge mobilization using submerged liquid jets is feasible in tanks of this configuration, and to estimate mixing times required to approach homogeneity of the contents of the tanks.

During the first phase of this work, numerical modeling considerations were investigated relative to measured MVST W-28 sludge properties. These considerations include time-step requirements for stable numerical solutions using a power law non-Newtonian model, grid resolution for the submerged liquid jets, and turbulent jet mixing effects. Results are presented which demonstrate the feasibility of computer modeling of the mixing and mobilization processes.

A two-parameter power-law model was extended to account for relative solids concentration variations spanning the range from fully settled W-28 sludge to clear supernatant. This was done by considering the consistency factor and the behavior index in the power law formulation to be functions of relative concentration of solids in the liquid. Computational results in a 1/6-scale half-filled tank with an initially settled sludge layer are presented. Simulations were conducted at $1 / 6$ scale to be compatible with planned experiments. Submerged 1-inch diameter jets with jet velocities from 5 to $20 \mathrm{ft} / \mathrm{sec}$ were modeled. Mobilization was successful at the highest jet velocities, but a sludge bank near the end of the tank persisted at the lower velocities. These results remain to be confirmed through experimental testing pianned at ORNL using a sludge simulant. Figure S-1 presents a schematic of the computational jomain of this problem computed. Figure S-2 presents a composite of the results of these simulations. The darkened areas in these figures represents the sludge layer.

In the second phase of this work, tank mixing times were computed in 1/6- and 2/3-scale tanks. Computer model conditions were consistent with planned experiments in scaled tanks at ORNL. Single- and double-jet mixing times were investigated for jets located at one-fourth the distance along the tank length. Saltwater in water was used as a tracer, and conductivity probes monitored the salt concentration at discrete locations. Mixing times in the computer simulations were based on time-history curves of concentration at discrete points in the tank 
and in the pump recirculation line. Mixing times in the 1/6-scale tank are in reasonable agreement with preliminary experimental data although the data exhibit a wide range of scatter.

Initial mixing times in $2 / 3$-scale simulations presented a counter-intuitive result in that mixing time at high jet velocity $(10 \mathrm{ft} / \mathrm{sec})$ with two jets was greater than that achieved at the same jet velocity with only one jet. This observation has also been qualitatively confirmed with two jet experiments at $1 / 6$ scale. Further numerical investigation showed it to be the result of two effects: one being the positional relationship of the initial source term of saltwater relative to the recirculation intake; the other being the actual criterion for mixing time. The latter was investigated further, and criteria were defined for global and truncated mixing time. It was determined that estimates of mixing time could vary by as much as $30 \%$ to $40 \%$, depending on how the mixing time is defined relative to the time history curves at a finite number of discrete locations. It is concluded that this result may help explain variations in mixing times determined from experimental correlations. It is further concluded that satisfactory comparison of numerically predicted mixing times with experimental data must be done using a consistent criterion.

A simulant development effort was undertaken to support the scaled tests. The objective was to develop a simulant which approximated the rheological characteristics of ORNL W-28 sludge. Various combinations of kaolin and bentonite in water were tested. These particulate materials are readily obtainable and readily disposable. A recommendation for a simulant is made. 


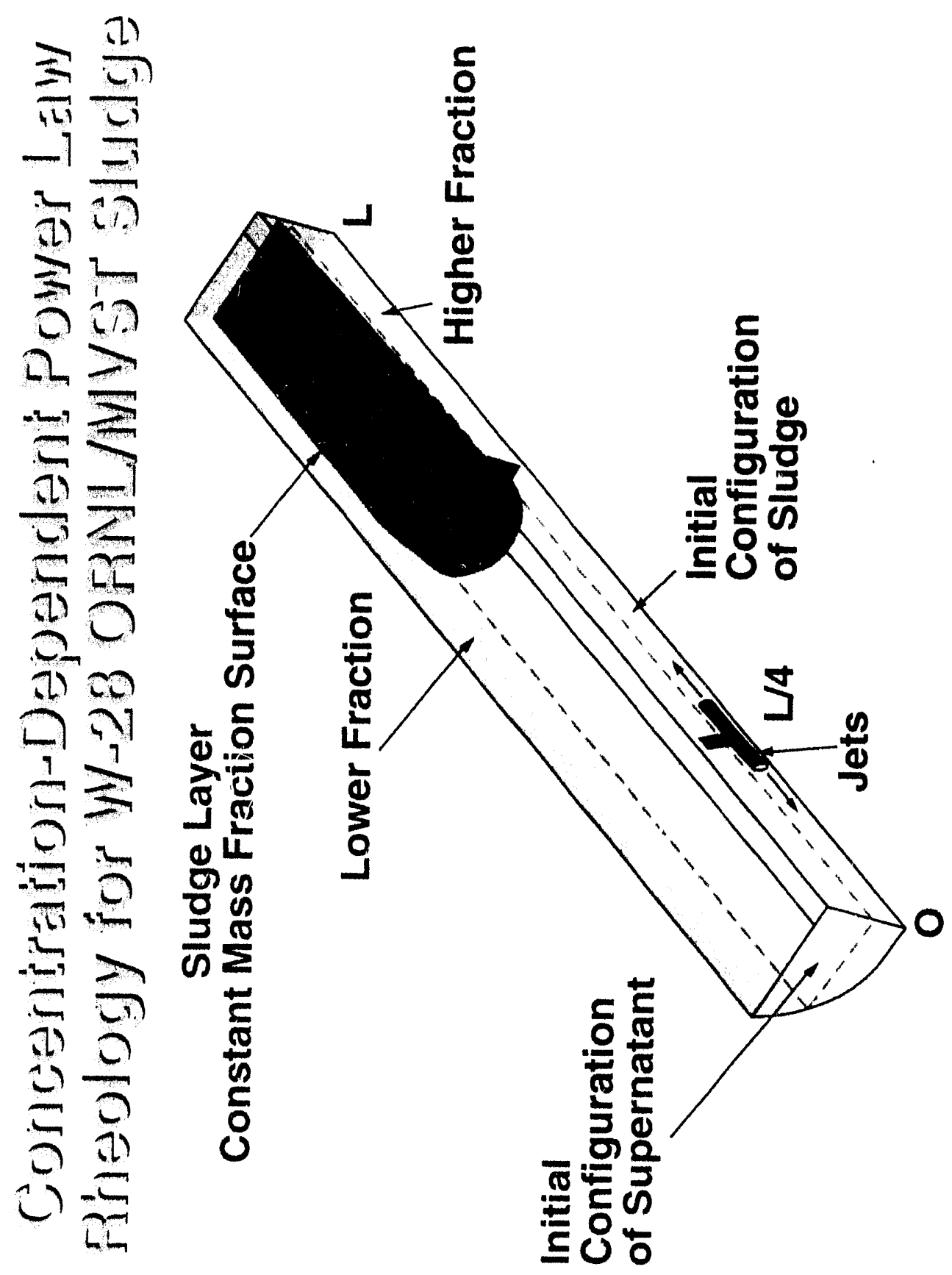

$\frac{8}{8}$

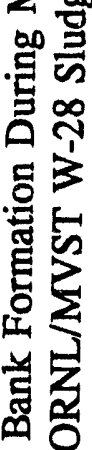

잉

흘

욤

o

灵

ธ논

.

四

웡

๘

ఫ्ठ

굴

줄

음

폭

롱

范范

के

馜 


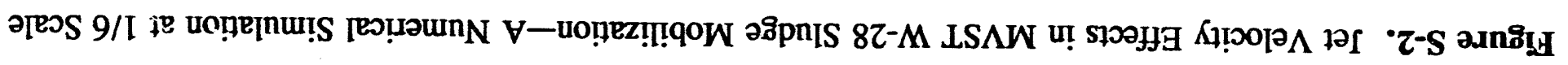

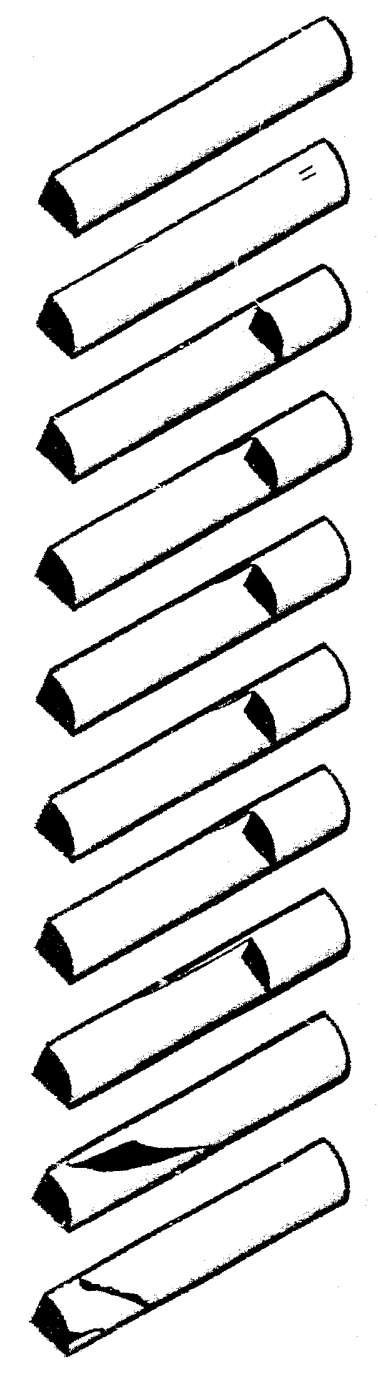

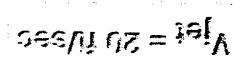

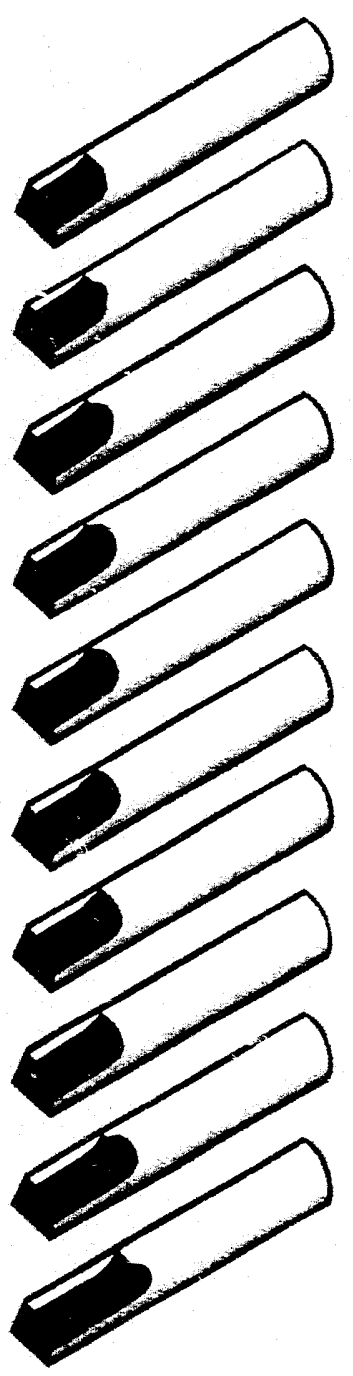

$\operatorname{ses} / A s s_{1}=20 / A$

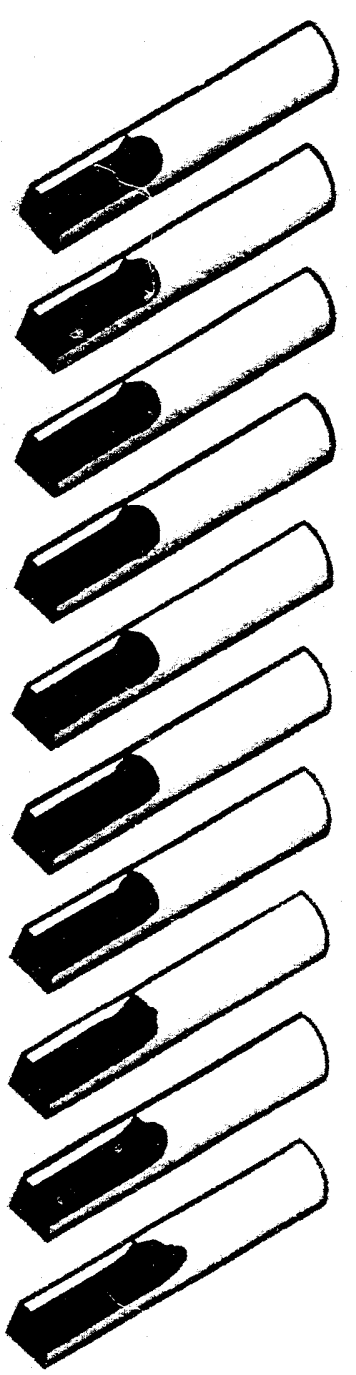

assntgh $=$ if $I_{h}$

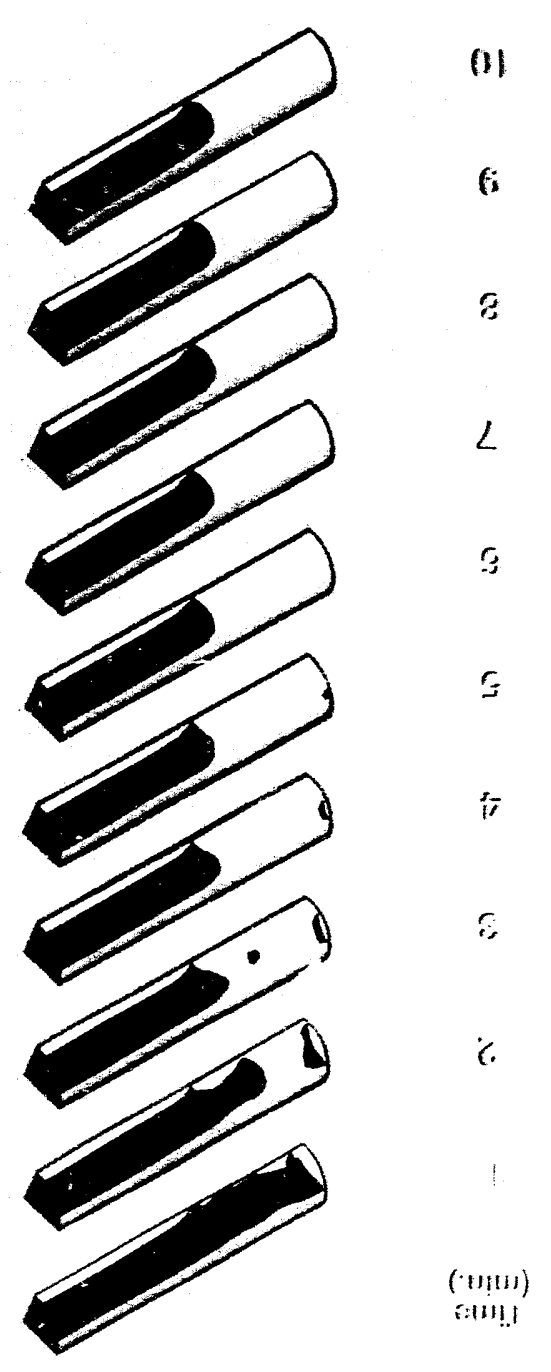

$\operatorname{sen} s=x$ 


\section{Acknowledgments}

This work was conducted under P.O. 10X-SL490V (J. J. Perona, Technical Contact) for Oak Ridge National Laboratory, operated by Martin Marietta Energy Systems for the U.S. Department of Energy under contract DE-AC05-840R2 1400. The work was conducted by Pacific Northwest Laboratory, operated by Battelle Memorial Institute for the U.S. Department of Energy under contract DE-AC06-76RLO 1830.

Simulant development and rheology measurements were conducted by M. R. Elmore, Waste Technology Center, Pacific Northwest Laboratory, Richland, Washington. 


\section{Contents}

Summary $\ldots \ldots \ldots \ldots \ldots \ldots \ldots \ldots \ldots \ldots \ldots \ldots \ldots \ldots \ldots \ldots \ldots \ldots$ iii

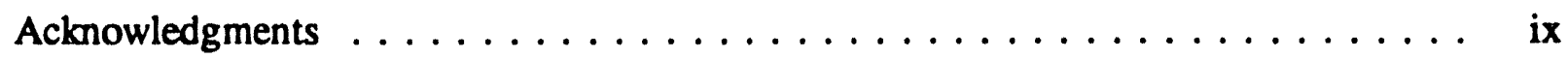

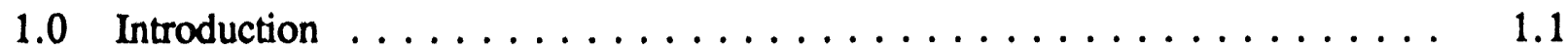

1.1 Objectives/Statement of Work . . . . . . . . . .

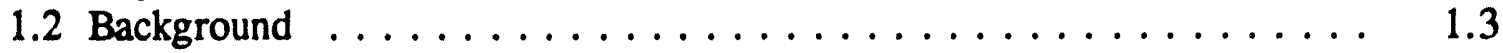

1.3 Motivation $\ldots \ldots \ldots \ldots \ldots \ldots \ldots \ldots \ldots \ldots \ldots \ldots \ldots \ldots \ldots \ldots . .4$

2.0 Conclusions and Recommendations $\ldots \ldots \ldots \ldots \ldots \ldots \ldots \ldots \ldots \ldots . \ldots \ldots$

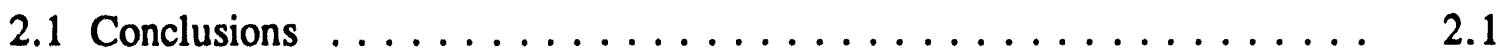

2.2 Recommendations .................... 2.2

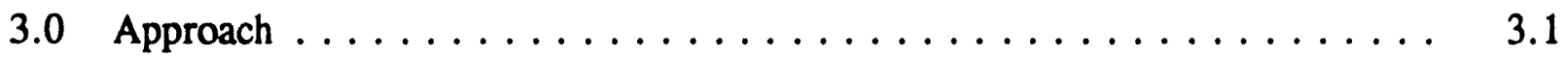

4.0 Feasibility of Mixing Simulations $\ldots \ldots \ldots \ldots \ldots \ldots \ldots \ldots . \ldots .1$

4.1 Concentration Dependent Rheological Model for

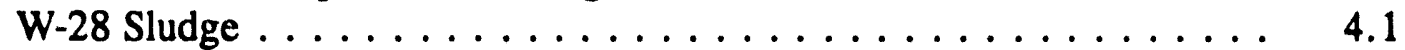

4.1.1 Data Review ...................... 4.1

4.1.2 Mathematical Basis . . . . . . . . . . . . . . 4.2

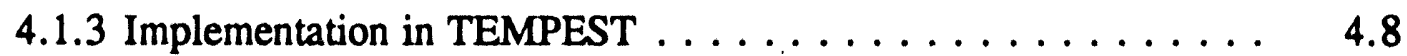

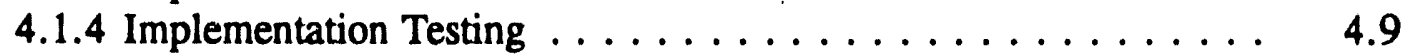

4.2 Time-Step Stability . . . . . . . . . . . . . . . 4.11

4.2.1 Mathematical Basis/Numerical Considerations . . . . . . . 4.11

4.2.2 Test Problem: Axisymmetric Plug Flow and Free Jet . . . . . . 4.12

4.3 Alternative Rheological Approaches . . . . . . . . . . . . . 4.17

5.0 Sludge Mixing Simulations in a Half-Filled $1 / 6-$ Scale Tank $\ldots \ldots \ldots \ldots .1$

5.1 Description of Models . . . . . . . . . . . . . . . 5.2

5.2 High Viscosity Newtonian Sludge Mixing Simulations . . . . . . . . 5.3

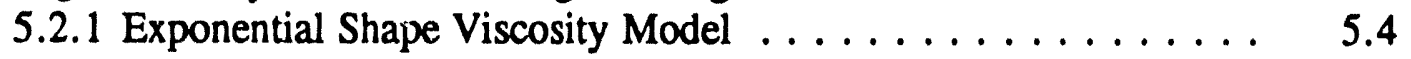

5.2 .2 Power Shape Viscosity Model . . . . . . . . . . . . 5.12

5.3 Non-Newtonian Sludge Mixing Simulations . . . . . . . . . . 5.22

6.0 Saline Solution Mixing Simulations in a Filled Tank $\ldots \ldots \ldots . \ldots . \ldots 6$

6.1 Mixing Time Criteria $\ldots \ldots \ldots \ldots \ldots \ldots \ldots \ldots . \ldots \ldots . \ldots \ldots$

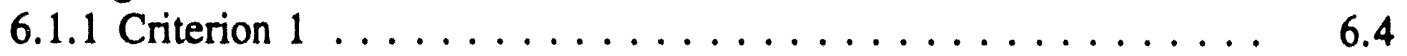

6.1 .2 Criterion $2 \ldots \ldots \ldots \ldots \ldots \ldots \ldots \ldots \ldots \ldots \ldots . \ldots \ldots$

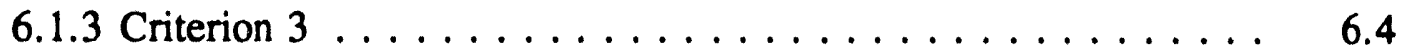

6.2 Time Dependent Concentration Curves . . . . . . . . . 6.5 
6.3 Mixing Time Results $\ldots \ldots \ldots \ldots \ldots \ldots \ldots \ldots \ldots$

6.3.1 Single Jet Simulations in a $1 / 6$ Scale Tank . . . . . . . . . . . . . 6.6

6.3.2 Single Jet Simulations in a $2 / 3$ Scale Tank . . . . . . . . . . . . . 6.12

6.3.3 Double Jet Simulations in a $2 / 3$ Scale Tank . . . . . . . . . . . 6.13

6.4 Comparison Between Single and Double Jet Mixing . . . . . . . . . . 6.19

7.0 Simulant Development $\ldots \ldots \ldots \ldots \ldots \ldots \ldots \ldots \ldots \ldots \ldots \ldots \ldots$

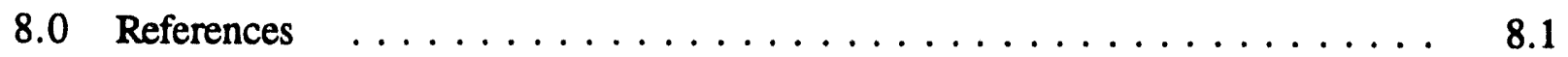




\section{Figures}

S.1 Computational Model Schematic Showing a Sludge Bank Formation During Mobilization of Concentration-Dependent Power Law Rheology ORNL/MVST W-28 Sludge . .................. v

S.2 Jet Velocity Effects in MVST W-28 Sludge Mobilization-A Numerical Simulation at $1 / 6$ Scale $\ldots \ldots \ldots \ldots \ldots \ldots \ldots \ldots \ldots \ldots \ldots \ldots$ vii

4.1 Comparison Between Rheological Data from a 1:1 Dilution Ratio of W-28 Sludge with a Power Law Fit for Two Sets of Parameters . . . . . 4.2

4.2 Experimental ORNL W-28 Data and Fitted Curve for the Consistency Factor Dependence on Relative Concentration $\ldots \ldots \ldots \ldots \ldots \ldots .4 .4$

4.3. Experimental ORNL W-28 Data and Fitted Five Parameter Curve for the Behavior index Dependence on Relative Concentration $\ldots \ldots \ldots$

4.4. Experimental ORNL W-28 Data and Fitted Two Parameter Curve for the Behavior Index Dependence on Relative Concentration

4.5. Velocity and Concentration Fields Between Parallel Plates Used to Indirectly Construct Rheograms with TEMPEST . . . . . . . . . .

4.6 Comparison Between Rheological Data from Various Dilution Ratios of W-28 Sludge with Rheograms Numerically Generated by TEMPEST . . . . .

4.7 Concentration Effects on the Time-Step Limiters and CPU Time for a Free Submerged Axisymmetric Jet Discharging Homogeneous W-28 Sludge . . . .

4.8 Numerically Stable Average Time-Step Size Variation as a Function of the Explicit Time-Step Multiplier for a Free Submerged Axisymmetric Jet Discharging Pure Non-Newtonian Sludge $\ldots \ldots \ldots \ldots \ldots \ldots \ldots$

4.9 Comparison of Velocity Magnitude Contours for Pure Non-Newtonian Sludge Computed with Different Time-Step Sizes and Initial Conditions . . . . . . . .

4.10 Experimental Data of Effective Viscosity as a Function of Strain Rate for a Pseudoplastic Fluid $\ldots \ldots \ldots \ldots \ldots \ldots \ldots \ldots$ 
5.1 Schematic Representation of the Computational Domain for the Melton Valley Storage Tanks . . . . . . . . . . . . . . .

5.2 Schematic of the Sampling Point Locations from Which Concentration Variations in Time were Monitored to Determine Mixing Times ......

5.3 Time Dependence of Solids Concentration for Neutrally Buoyant Particles in Water Without Concentration Dependent Viscosity (Case 1a, SG $=1$ ) . . .

5.4 Time Dependence of Solids Concentration for Neutrally Buoyant Particles in Water Without Concentration Dependent Viscosity (Case 1b, SG $=1.5$ ) .

5.5 Time Dependence of Solids Concentration for Neutrally Buoyant Particles in Water Without Concentration Dependent Viscosity (Case 1c, SG = 2) . .

5.6 Composite of the Time Variation of the Solids Concentration at the Modeled Recirculating Pump Intake Showing the Effect of Solids Density on Mixing Time (Cases 1a-1c) $\ldots \ldots \ldots \ldots \ldots \ldots \ldots \ldots$

5.7 Composite of the Time Variation of the Solids Concentration at the Modeled Recirculating Pump Intake Showing the Effect of Maximum Viscosity Ratio for High Molecular and Turbulent Diffusivity . . . . . . .

5.8 Time Dependence of Solids Concentration for Solid Particles in Water with an Exponential Viscosity Shape Function (Case 3b) $\ldots \ldots \ldots \ldots \ldots$

5.9 Time Dependence of Solids Concentration for Solid Particles in Water with an Exponential Viscosity Shape Function (Case 3c) $\ldots \ldots \ldots \ldots . . .5 .14$

5.10 Time Dependence of Solids Concentration for Solid Particles in Water with an Exponential Viscosity Shape Function (Case 3d) . . . . . . . .

5.11 Time Dependence of Solids Concentration for Solid Particles in Water with an Exponential Viscosity Shape Function (Case 4d) $\ldots \ldots \ldots \ldots$

5.12 Composite of Time Dependence of Solids Concentration at Modeling Recirculating Pump Intake (Cases 1c, 3b-3d, and 4d)

5.13 Volume Fraction as a Function of Time for a Concentration Dependent Power Shape Newtonian Viscosity Model with Dual Jet Velocity of $1 \mathrm{ft} / \mathrm{sec} . . .$. . 
5.14 Mixing Times as a Function of Jet Velocity for a Power-Shape Newtonian-Viscosity Model $\ldots \ldots \ldots \ldots \ldots \ldots \ldots$

5.15 Volume Fraction as a Function of Time for a Concentration-Dependent Power-Law Model for the W-28 Sludge Waste with Dual Jet Velocity of $5 \mathrm{ft} / \mathrm{sec}$

5.16 Volume Fraction as a Function of Time for a Concentration-Dependent Power-Law Model for the W-28 Sludge Waste with Dual Jet Velocity

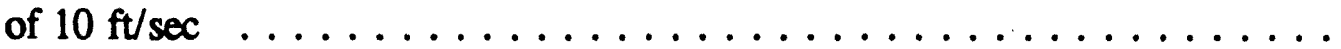

5.17 Volume Fraction as a Function of Time for a Concentration-Dependent Power-Law Model for the W-28 Sludge Waste with Dual Jet Velocity

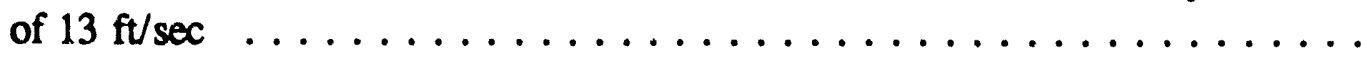

5.18 Volume Fraction as a Function of Time for a Concentration-Dependent Power-Law Model for the W-28 Sludge Waste with Dual Jet Velocity of $20 \mathrm{ft} / \mathrm{sec}$ Where Mixing Throughout the Tank has been Reached . . . . . .

5.19 Time Sequence of Velocity Fields and Mass Fraction Contours on the Plane of Symmetry of the 1/6-Scale Tank for a Concentration-Dependent Power-Law Model for the W-28 Sludge Waste with Dual Jet Velocity of $5 \mathrm{ft} / \mathrm{sec} \ldots \ldots$.

5.20a Time Sequence of Velocity Fields and Mass Fraction Contours on the Plane of Symmetry of the 1/6-Scale Tank for a Concentration-Dependent Power-Law Model for the W-28 Sludge Waste with Dual Jet Velocity of $10 \mathrm{ft} / \mathrm{sec}$. . . . .

5.20b Time Sequence of Velocity Fields and Mass Fraction Contours on the Plane of Symmetry of the 1/6-Scale Tank for a Concentration-Dependent Power-Law Model for the W-28 Sludge Waste with Dual Jet Velocity of $10 \mathrm{ft} / \mathrm{sec} . \ldots$.

5.21a Time Sequence of Velocity Fields and Mass Fraction Contours on the Plane of Symmetry of the 1/6-Scale Tank for a Concentration-Dependent Power-Law Model for the W-28 Sludge Waste with Dual Jet Velocity of $13 \mathrm{ft} / \mathrm{sec} \ldots \ldots$

5.21b Time Sequence of Velocity Fields and Mass Fraction Contours on the Plane of Symmetry of the 1/6-Scale Tank for a Concentration-Dependent Power-Law Model for the W-28 Sludge Waste with Dual Jet Velocity of $13 \mathrm{ft} / \mathrm{sec} \ldots \ldots$ 
5.22 Time Sequence of Velocity Fields and Mass Fraction Contours on the Plane of Symmetry of the 1/6-Scale Tank for a Concentration-Dependent Power-Law Model for the W-28 Sludge Waste with Dual Jet Velocity of $20 \mathrm{ft} / \mathrm{sec} \ldots \ldots$

5.23 Jet Velocity Effects in MVST W-28 Sludge Mobilization-A Numerical

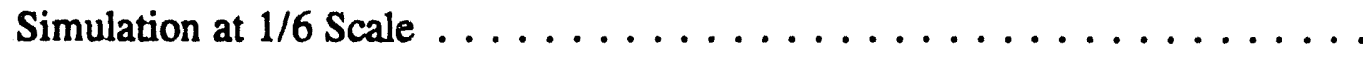

6.1 Examples of Concentration Curves Which Rapidly Overshoot the Average Concentration and Then Oscillate Toward It $\ldots \ldots \ldots \ldots \ldots \ldots$

6.2 Examples of Concentration Curves Which Slightly Overshoot the Average Concentration . . . . . . . . . . . . . . . . . . . . .

6.3 Typical Time Dependent Concentration Curves for Double Jet Mixing at High Velocity in a $2 / 3$-Scale Tank . . . . . . . . . . . . . .

6.4 Typical Time Dependent Concentration Curves for Single Jet Mixing at Moderate Velocity in a $2 / 3$-Scale Tank . . . . . . . . . . . . .

6.5 Comparison of Experimental (ORNL), Numerical (TEMPEST), and Correlated (Okita and Oyama) Mixing Time Values for a Filled 1/6-Scale Tank with Water $\ldots \ldots \ldots \ldots \ldots \ldots \ldots \ldots \ldots$

6.6 Single Jet Mixing Times as a Function of Jet Velocity for a 2/3-Scale Tank According to Three Different Mixing Criteria . . . . . . . . . .

6.7 Single Jet Mixing Times as a Function of the Percent Departure from the Average Concentration for a $2 / 3$-Scale Tank . . . . . . . . . . . .

6.8 Comparison of the Axial Velocity Along the Jet Axis for Single and Double Jet Arrangements in a $2 / 3$-Scale Tank . . . . . . . . . . . . . .

6.9 Double Jet Mixing Times as a Function of Jet Velocity for a 2/3-Scale Tank According to Three Different Mixing Criteria . . . . . . . . . . . . . .

5.10 Double Jet Mixing Times as a Function of the Percent Departure from the Average Concentration for a $2 / 3$-Scale Tank . . . . . . . . . . . . 
6.11 Comparison of Mixing Times as a Function of Flow Rate for Several Percentage Departures from the Average Concentration Based on Criterion 1 for Single and Double Jet Mixing in a 2/3-Scale Tank . . . . 6.20

7.1 Shear Stress Versus Shear Rate Plot for $\mathbf{1 5} \mathrm{wt} \%$ Kaolin in Water Slurry . . 7.4

7.2 Shear Stress Versus Shear Rate Plot for $15 \mathrm{wt} \%$ Kaolin in $1 \mathrm{wt} \% \mathrm{NaCl}$ Solution $\ldots \ldots \ldots \ldots \ldots \ldots \ldots \ldots \ldots \ldots \ldots \ldots . \ldots \ldots$

7.3 Shear Stress Versus Rate Plot for 15 wt\% Kaolin in Water Slurry $\ldots \ldots .7 .6$

7.4 Shear Stress Versus Rate Plot for 15 wt\% Kaolin in Water Slurry . . . . . . 7.7

7.5 Comparison of the Effective Average Viscosity as a Function of Concentration Ratio Between the W-28 Waste Sludge and the Kaolin-Based Simulant . . . . . . . . . . . . . . . . . . . 7.9 


\section{Tables}

4.1 Power Law Model Parameters as a Function of Relative

Concentration for the W-28 Waste Sludge $\ldots \ldots \ldots \ldots \ldots \ldots \ldots .4 .4$

5.1 Mixing Simulation Parameters for an Exponential Shape

Viscosity Model in a Half-Filled $1 / 6-$ Scale Tank . . . . . . . . 5.5

6.1 Comparison of Dimension Ratios Between Scaled Tanks $\ldots \ldots \ldots \ldots .6 .13$

6.2 Single Jet Mixing Times in Water for a $2 / 3$-Scale Tank $\ldots \ldots \ldots \ldots .6 .13$

6.3 Effect of Percent Deviation from Average Concentration

on Estimated Single Jet Mixing Times in a 2/3-Scale Tank . . . . . . . 6.14

6.4 Double Jet Mixing Times in Water for a $2 / 3$-Scale Tank . . . . . . . . 6.17

6.5 Effect of Percent Deviation from Average Concentration

on Estimated Double Jet Mixing Times in a 2/3-Scale Tank . . . . . . 6.18

7.1 Settled Solids Behavior for Kaolin Clay Slurries . . . . . . . . . . 7.3

7.2 Curve Fit Coefficients Using Power-Law Model for 15 wt\% Kaolin

Slurries in Water and in $1 \mathrm{wt} \% \mathrm{NaCl}$ Slurries . . . . . . . . . . 7.8

xviii 


\subsection{Introduction}

The mobilization and retrieval of radioactive sludges that are stored in tanks constitutes a prevalent problem at several U.S. Department of Energy sites. Before the transuranic waste slurries can be transported from any tank to a processing plant to be transformed into stable solids, the supernatant and the sludge must be thoroughly mixed (mobilized and dispersed) within the storage tanks. One proposed approach to achieve sludge mobilization in tanks is to use submerged jets because of the simplicity of the design, reduced safety issues, and low cost. This report focuses on mixing and mobilization using submerged liquid jets in configurations similar to the Melton Valley Storage Tanks (MVST) at Oak Ridge National Laboratory (ORNL).

The MVSTs are horizontal, cylindrically shaped tanks, with rounded ends. They are nominally $60 \mathrm{ft}$ long, $12 \mathrm{ft}$ in diameter, and 50,000 gal capacity. Contents of the tanks vary; but nominally they contain a settled sludge layer covered ty a layer of supernatant. Sludge and supernatant depths vary. Ceo et al. (1990) report physical characterization data for sludge samples. These data indicate that the sludge in both its settled state and in a diluted state exhibits concentration-dependent non-Newtonian rheological characteristics.

Computer modeling of mobilization and mixing in horizontal, cylindrical storage tanks using submerged liquid jets is the subject of this report. The computer modeling uses the TEMPEST computational fluid dynamics computer program (Trent and Eyler 1992). The eventual goals of the simulations are to determine under what conditions sludge mobilization using submerged liquid jets is feasible in tanks of this configuration and to estimate mixing times required to approach homogeneity of the contents of the tanks.

During the first phase of this work, numerical modeling considerations were investigated relative to measured MVST W-28 sludge properties. Results of this phase are presented in Section 4.0. Numerical considerations investigated include time-step requirements for stable numerical solutions, use of a power law non-Newtonian model, grid resolution for submerged liquid jets, and turbulent jet mixing effects. Results are presented which demonstrate the feasibility of computer modeling of the mobilization and mixing processes.

A two-parameter power-law model was extended to account for relative concentration variations spanning the range from fully settled W-28 sludge to clear supernatant. This was done by considering, the consistency factor and the behavior index in the power law formulation to be functions of relative concentration of solids in the liquid. 
Section 5.0 of this report presents computational results in a 1/6-scale half-filled tank with an initially settled sludge layer. The concentration-dependent non-Newtonian power law model of W-28 sludge is used in this calculation. Submerged 1-in.-diameter jets with jet velocities from 5 to $20 \mathrm{ft} / \mathrm{sec}$ were modeled. Mobilization was accomplished at the highest jet velocities, but a sludge bank near the end of the tank persisted at the lower velocities. These results remain to be confirmed through experimental testing planned at ORNL using a sludge simulant.

Section 6.0 presents the second phase of this work. Tank mixing times were computed in 1/6- and 2/3-scale tanks. Computer model conditions were consistent with planned experiments in scaled tanks at ORNL. Single- and double-jet mixing times were investigated for jets located at one-quarter of the tank length along the horizontal. Saltwater in water was used as a tracer. Conductivity probes at fixed locations measured concentration of salt. Mixing times in the computer simulations were based on time-history curves of concentration at discrete points in the tank and in the pump recirculation line. Mixing times in the 1/6-scale lank are in reasonable agreement with preliminary experimental data, although the data exhibit a wide range of scatter.

In preparation for ORNL experiments, a simulant development effort was undertaken. The objective was to develop a simulant which approximated rheological characteristics of W-28 sludge. Testing was done with various combinations of kaolin and bentonite in water. These are readily obtainable and readily disposable particulate materials. A recommendation for a simulant is made. The simulant development results are presented in Section 7.0 of this report.

\subsection{Objectives/Statement of Work}

The purpose of this work is to assess the feasibility of performing computer simulations of submerged-jet-mobilization with non-Newtonian fluids, to estimate the time required to reach homogeneous concentration distributions in tanks, and to provide a recipe for a sludge simulant. Simulations in tank configurations similar to the ORNL storage tanks, known 's MVSTs, are carried out with the three-dimensional time-dependent fluid flow simulator program TEMPEST (Trent and Eyler 1992). Simulations are intended to complement experimental work at ORNL in 1/6- and 2/3-scale MVST geometries and to support investigations in the retrieval of wastes in the full-scale MVST. Initial phases of this work include the mixing of a saline solution in tanks filled with water. 
Specific objectives of the present work are to:

- Investigate requirements for stable numerical solutions of submerged liquid jet mixing in a non-Newtonian fluid whose rheology is described by a concentration-dependent powf; law model approximating $W^{\top}-28$ sludge.

- Simulate mobilization and homogenization of sludge lajers of Newtonian and nonNewtonian fluids in a half-filled $1 / 6$-scale tank.

- Investigate definition and application of mixing time criteria based on time-history concentration curves.

- Compute mixing of saltwater in water in 1/6- and 2/3-scale tanks.

- Investigate and recommend a kaolin-based simulant for W-28 sludge to be used in further experimental studies at $1 / 6$ and $2 / 3$ scale.

\subsection{Background}

The systematic study of jet mixing in tanks started half a century ago with the work of Fossett and Prosser (1949) who were interested in the mixing of fuels. Since that time, experimental and theoretical studies involving various tank geometries and jet axis orientations have been analyzed. The bulk of research in this area has concentrated on the mixing of emulsions and dilute suspensions, which are principally Newtonian fluids. The preferred geometric configuration in these investigations is the vertical cylindrical tank using a single submerged jet whose location and orientation within the tank are varied. Research in this area has been reviewed by Rice (1986), Maruyama (1986), and Tatterson (1991). However, the study of jet mixing in tanks containing highly viscous and non-Newtonian fluids has not received much attention.

Oak Ridge researchers have recently performed investigations on mobilization of sludge similar to the sludge in the MVSTs. Shor and Cummins (1991) used a single-point sluicing technique in which several sludge simulants were tested. They concluded that their technique requires a maneuverable nozzle and that the pump suction point should be close to the sludge. They also pointed out that the mobilization process is more effective when the supernatant layer is thin. Further experiments are being performed using other techniques, and larger scale tests have also been planned. 
In an effort to provide support for research and development activities related to the design of retrieval and transport systems to handle the radioactive slurries, Ceo et al. (1990) experimentaily determined several physical properties of various sludge samples from eight storage tanks at ORNL. Their rheological measurements showed that sludge and slurries behave like viscoplastic materials with a small apparent yield stress (less than that of mayonnaise). Once the yield stress has been exceeded, the sludges exhibit pseudoplastic behavior. Their data were later used by Youngblood et al. (1991) to correlate sludge transport behavior and to calculate the pressure drop in pipes. They reported that either the Bingham plastic model or the power law (Ostivald de Waele) model could be used to fit the rheological data. Graphical comparisons indicate that the power law model renders a better description of the stress/strain rate data, provided the strain rate (shear rate) exceeds $1.0 \mathrm{sec}^{-1}$.

To the best of our knowledge, no correlations have been specifically developed for the mixing of either Newtonian or non-Newtonian fluids in horizontal cylindrical tanks. Some investigators believe that the tank geometry is not very important in determining the mixing time. However, there is no conclusive information on the effect of the vessel geometry on submerged jet mixing. Recently, several mixing experiments of water in a 1/6-scale tank at ORNL have raised several questions about the uniqueness of the mixing time.

The issue of a criterion for mixing has been treated rather vaguely in the literature. In some instances mixing has been defined in a rather qualitative manner. Quantitative mixing criteria are based on the concentration information obtained from very arbitrarily chosen monitor locations. Some mixing time correlations have been based on the concentration information recorded at only two points within the vessel (Maruyama 1986). This issue has been addressed by showing that the particular definition of mixing markedly affects the mixing time. The inherent non-uniqueness of mixing times can be traced to the lack of generality of the criterion used.

\subsection{Motivation}

State-of-the-art nonintrusive fluid flow measuring techniques (e.g., laser Doppler and particle image velocimetry) cannot be applied to most physical waste simulants because they are generally opaque. An additional shortcoming of experimentation is that, even though a qualitative scenario of the phenomena is gained, scale-up from a model to a prototype is not straightforward when dealing with non-Newtonian fluids. Dimensionless numbers based on physical properties of non-Newtonian fluids are generally not as meaningful as they are in Newtonian fluid mechanics, because of the nonlinear dependency of stress on strain rate. Non-Newtonian dimensionless groups can vary in time and in space over the entire fluid domain. In addition, waste simulants may not fully mimic the behavior of actual radioactive 
wastes. Hence, experimental results in which the working fluid is a simulant whose properties do not fully match the rheology of real waste slurries may not reveal all of the details required to design full-scale prototypes.

For several years, the TEMPEST code has been used extensively in the simulation of three-dimensional time-dependent thermo-fluid problems in complex geometries. Several Newtonian sludge mobilization calculations in tanks have been successfully performed. The computed velocity field and shear stress distributions at the tank floor were found to be in good agreement with earlier experimental work (Rajaratnam 1976). The TEMPEST capabilities have been recently extended to handle the flow of generalized Newtonian (pseudoplastic and yield-pseudoplastic) fluids. The power-law model was implemented and validated for pipe and channel flow geometries for which analytic solutions are known. TEMPEST has been used extensively in recent years to support tank mixing studies and application to waste storage tanks at DOE's Hanford Site (Bamberger et al. 1991, 1993; Eyler and Michener 1992).

Numerical simulations assist in gaining a more fundamental understanding of the detailed phenomena occurring in the mobilization process. They not only complement the ongoing experimental efforts, but also provide information which cannot be obtained from experimentation. Through numerical experiments it is possible to construct accurate correlations for mixing times, because the concentration time history of each computational cell can be computed. 


\subsection{Conclusions and Recommendations}

The goals of this work were achieved. Investigations to model mixing and mobilization of Newtonian and non-Newtonian sludge mixtures in a horizontal cylindrical tank with submerged liquid jets were completed. The investigations included time-step stability limitations arising from the occurrence of infinite effective viscosity at zero shear stress in a power-law fluid; effects of the grid resolution structure on mixing; turbulent diffusivity considerations; and the effects of jet velocity. Saltwater mixing simulations similar to planned experiments at ORNL were also completed in $1 / 6$ - and $2 / 3$-scale tanks.

\subsection{Conclusions}

Preliminary investigations were conducted to investigate the feasibility of numerically simulating mobilization and mixing with submerged liquid jets in horizontal cylindrical storage tanks, with the following conclusions:

- Numerical stability time-step limitations do not overly restrict time-dependent mixing computations. Such a concern arises from the fatalistic character of a power law fluid model, which exhibits an infinite effective viscosity at zero strain rate and possibly very large effective viscosities at strain rates.

- Grid structure resolution can affect mixing. The resolution must be adequate to resolve jet entrainment and jet spread. These phenomena are key to accurately modeling the mixing process.

- Cylindrical (polar) coordinates are not suitable for fully filled tank mixing simulations. The centerline (tank axis) induces an excessive numerical diffusion during mixing.

Computer simulations of sludge mobilizing and mixing were conducted for concentration dependent Newtonian and non-Newtonian fluid models, with the following conclusions:

- Mobilization and mixing simulations with TEMPEST yielded stable, consistent solutions for both concentration-dependent Newtonian sludges and for power law rheology mixtures with concentration-dependent consistency factor and behavior index.

- Mobilization simulation of W-28 sludge in a half-full 1/6-scale tank with 1-in. diameter jets predicted complete mixing for jet velocities greater than about $13 \mathrm{ft} / \mathrm{sec}$. This result remains to be confirmed experimentally. 
Computer simulations of saltwater-in-water mixing in filled 1/6- and 2/3-scale tanks were completed. Submerged liquid jets located at one-quarter of the distance along the tank length were used to approximate experiments being conducted at ORNL at 1/6-scale, and planned at 2/3- scale. Conclusions reached include:

- The criterion used to determine mixing time is significant. Comparison of mixing time results computed with different defined criteria, using the same time-history data, can result in as much as a 30 to $40 \%$ variation in estimated mixing time. This is significant in comparing computer simulations to experimental data and may, in part, be an explanation for the wide variation in empirical mixing correlations.

Investigations were conducted to develop a simulant for W-28 sludge which could be used in scaled experiments. Conclusions reached include:

- The exact rheological character of W-28 sludge could not be replicated. An approximate model fluid which exhibits similar features can be made using a kaolin in water mixture. The material is readily obtainable, relatively inexpensive, and is not overly difficult to dispose of.

\subsection{Recommendations}

Several recommendations are offered as a result of this work.

- Alternative rheological models to the power law approach should be investigated to determine if ORNL waste tank sludges can be better represented. This may be important to wastes other than W-28.

- Mixing time criteria should be evaluated further to determine their significance to mixing time estimates in nonhomogeneous situations (i.e., sludge layer mixing as compared to saltwater-in-water mixing).

- A more complete and critical comparison of computer predictions to ORNL-scale tank mixing experiments should be done when experimental data are available. 


\subsection{Approach}

The present investigation is a requisite in the modeling of sludge mobilization in full-scale tanks whose contents have the rheological properties of radioactive waste. First, an analytic constitutive equation is developed to reproduce the rheological properties of an actual waste slurry, within the range of experimentally measured strain rates. The correct implementation of the model into TEMPEST is then tested with a model problem, from which rheograms consistent with experimental data can be numerically computed. Ceo et al. (1990) indicated that data can be best fit with the power-law morel. Similarities among the rheologies of the various waste sludges of the ORNL W-series tanks indicate that the functional form of the concentration-dependent parameters should be essentially the same. In this study, mixing simulations with a non-Newtonian fluid are performed with the power-law approximation of the rheological properties of the W-28 ORNL sludge waste. Two functions are required to specify the rheological characteristics of this sludge, namely, concentration-dependent cousistency and concentration-dependent behavior index functions.

A critical issue in any complex numerical simulation is that of cost effective CPU time. Furthermore, the usefulness of numerical results is based on the accuracy of the solutions. These two issues are linked to the time-step required to achieve a numerically stable solution. To investigate these issues, a geometry was chosen in which an axisymmetric submerged free jet discharges into a homogeneous medium of a non-Newtonian power-law fluid. This test problem is representative of the jet mixing in the horizontal MVST. Strain rate values in the free jet problem are expected to be similar to those in the MVST geometry. Consequently, a good estimate of the computational resource requirements for the actual tank mobilization calculations can be inferred from the free jet simulation results.

For many years the power-law model has been accepted in engineering practice as a reasonably accurate way of modeling pseudoplastic fluids. However, it is well known that the power-law model fails to predict the correct shear stress at very low strain rates (less than $\left.1 \mathrm{sec}^{-1}\right)$. It has been argued that in most engineering applications low strain rates are not important. Nevertheless, in a time dependent computer code like TEMPEST in which simulations start from a motionless state, there will be regions in the computational domain with very low strain rates. Low strain rate regions will be numerically assigned high effective viscosities. Even though the effect in the final computed result may not be significant once steady state has been reached, these high effective viscosity regions might limit the time-step for stable solutions, and thus increase the required CPU time. This possible scenario was not a problem in the particular simulations that were carried out here. For more general computations, this problem can be overcome by implementing other rheological models. 
Based on various mixing time criteria, it was found that the mixing time strongly depends on the criteria used to determine it. A global mixing criterion cannot be practically implemented experimentally, because it requires knowledge of the time concentration history at all points in the fluid domain. Alternately, mixing times determined from truncated criteria (e.g., using a small number of discrete measurement locations) may not adequately represent the true mixing time over the whole domain. These effects are investigated using several criteria with consistent, continuous time-history curves in numerical experiments. 


\subsection{Feasibility of Mixing Simulations}

In this section the two-parameter power-law model is extended to account for relative volumetric concentration variations of the sludge. The particular functional forms of the consistency factor and the behavior index are based on experimental data for the W-28 waste of the MVST. The function coefficients were calculated by a least squares fit of available data. The concentration-dependent power-law model has been implemented and successfully validated in TEMPEST. Demonstration calculations for an axisymmetric submerged free jet are illustrated in the form of velocity magnitude contours. The working fluid in these simulations has the rheology of the waste in tank W-28, but the concentration is considered constant. Time-step limiters and stability are discussed for the free jet calculations, together with the impact these issues are likely to have in the full scale MVST sludge mobilization simulations. Drawbacks in using the power law model and possible modifications to the existing non-Newtonian models in TEMPEST are discussed.

\subsection{Concentration Dependent Rheological Model for W-28 Sludge}

Based on experimental data of the waste sludge in the MVST (Ceo et al. 1990), a functional form for a concentration-dependent power-law model is proposed. This rheological equation of state is implemented in TEMPEST and rheograms are constructed which are in excellent agreement with available data.

\subsubsection{Data Review}

Physical properties of the radioactive sludge wastes in the MVST (rheology, deilsity, sedimentation rate, and particle sizes) have been experimentally determined (Ceo et al. 1990). Rheological measurements were performed using a digital Brookfield rotational viscometer. The range of strain rates in the rheograms for the $\mathrm{W}-28$ sludge varied form 0.08 to $16.8 \mathrm{sec}^{-1}$. In this range, the sludge behaves as a yield pseudoplastic material. Youngblood et al. (1991) pointed out that measurements at shear rates on the order of $500 \mathrm{sec}^{-1}$ are necessary for accurate modeling. Unfortunately, the inherent difficulty of handling these samples has precluded measurements at high shear rates. Power-law and Bingham plastic constitutive equations were suggested as alternative models for the W-28 sludge. However, the power law model proved to fit the data better than the Bingham plastic model. Figure 4.1 shows experimental data points for a $1: 1$ dilution ratio (concentration ratio $=0.5$ ) of $\mathrm{W}-28$ wastes and the fitted points for two sets of power-law parameters. Comparison of the reported data with the power-law model shows excellent agreement, provided that strain rates are greater than $1 \mathrm{sec}^{-1}$. 


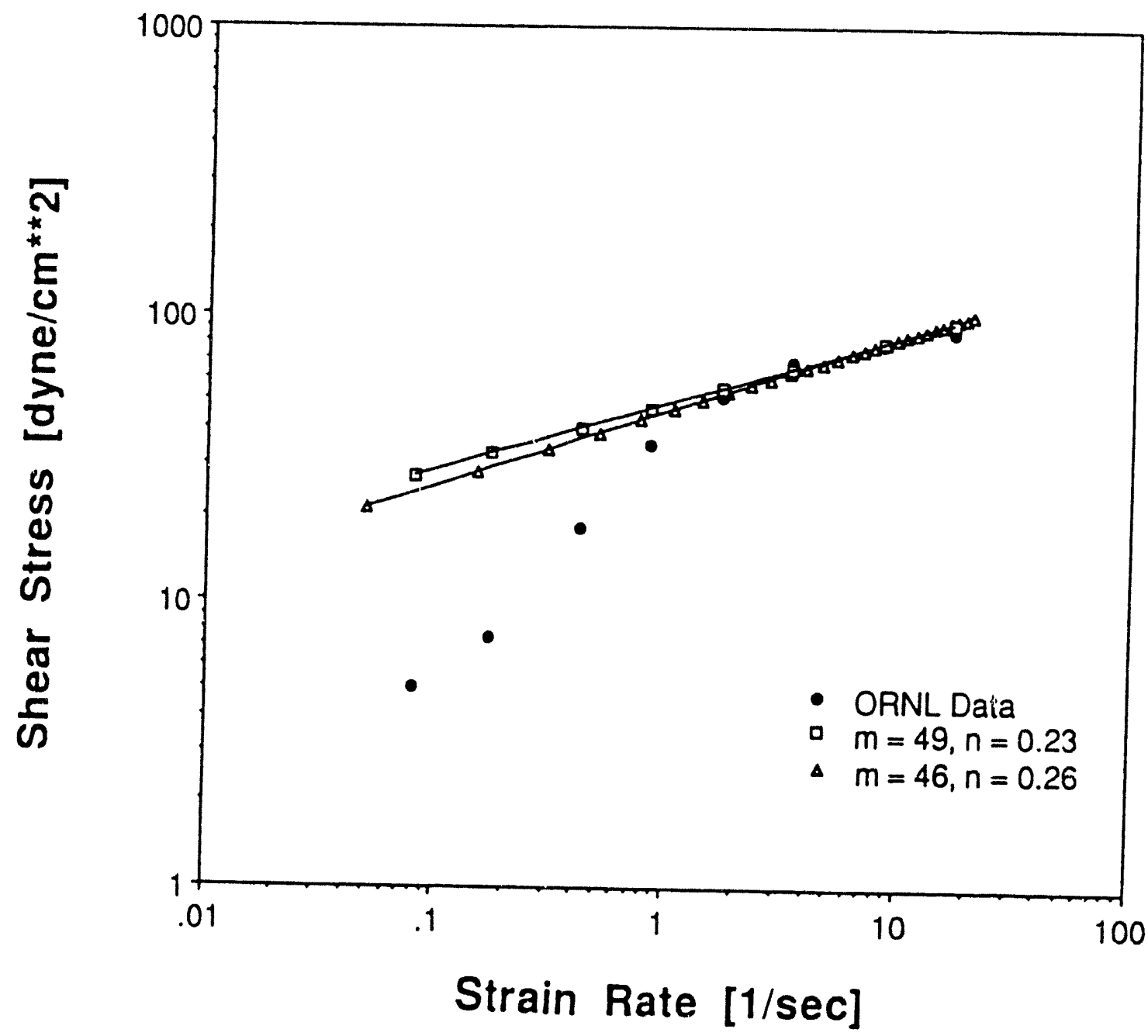

Figure 4.1. Comparison Between Rheological Data from a 1:1 Dilution Ratio of W-28 Sludge with a Power Law Fit for Two Sets of Parameters

\subsubsection{Mathematical Basis}

According to the power-law model, the shear stress $\tau$ in a homogeneous fluid is related to the strain rate $\dot{\gamma}$ through the equation

$$
\tau=\mathbf{m} \dot{\gamma}^{\mathrm{n}}
$$

where $\mathrm{m}$ is the consistency factor and $\mathrm{n}$ is the behavior index. During the mobilization of sludge in a tank, the concentration of species is no longer homogeneous. Therefore, the two 
power-law parameters (consistency factor and behavior index) have to be treated as concentration dependent functions. Equation (4.1) can be generalized into an equation of the form

$$
\tau=\mathrm{m}\left(\mathrm{C}_{\mathrm{r}}\right) \dot{\gamma}^{\mathrm{n}\left(\mathrm{C}_{\mathrm{r}}\right)}
$$

where $m\left(C_{r}\right)$ and $n\left(C_{r}\right)$ are functions of the volumetric concentration ratio $C_{r}$ in the slurry. This ratio is defined by

$$
\mathrm{C}_{\mathrm{r}}=\frac{\mathrm{C}_{\mathrm{v}}}{\mathrm{C}_{\mathrm{v}, \mathrm{m}}}
$$

where $C_{v}$ is the volumetric concentration of solids and $C_{v, m}$ is the maximum volumetric packing factor. During mobilization and mixing, $\mathrm{C}_{\mathrm{v}}$ will be space and time dependent. Thus, $\mathrm{C}_{\mathrm{r}}$ will vary from 0 to 1 . Reasonably simple functional forms for $m$ and $n$ that fit the available data are sought. Table 4.1 presents the ORNL W-28 sludge data (Ceo et al. 1990).

For this data set, the consistency factor $m$ is observed to increase monotonically with the relative concentration ratio, $\mathrm{C}_{\mathrm{r}}$. Thus, the four data points can be fit into the cubic function

$$
m\left(C_{r}\right)=A_{o}+A_{1} C_{r}+A_{2} C_{r}^{2}+A_{3} C_{r}^{3}
$$

The following coefficients were determined for the data in Table 4.1:

$$
\begin{aligned}
& A_{0}=0.0022 \\
& A_{1}=3.0513 \\
& A_{2}=17.631 \\
& A_{3}=-10.684
\end{aligned}
$$

A comparison of the data and the curve fit is presented in Figure 4.2.

The consistency factor $m$ in other non-Newtonian mixtures (e.g., clay suspensions, magnetite media, lignite in methanol) has also been found to exhibit monotonically increasing dependence with concentration (Govier and Aziz 1987, Darby 1986). However, the functional form was not necessarily a cubic polynomial. 
Table 4.1. Power Law Model Parameters as a Function of Relative Concentration for the W-28 Waste Sludge

\begin{tabular}{|c|c|c|}
\hline $\mathbf{C}_{\mathbf{r}}$ & $\mathbf{m ~}\left[\mathbf{P a ~ s e c} \mathbf{n}^{\mathbf{n}}\right.$ & $\mathbf{n}$ \\
\hline 0.00 & 0.0022 & 1.00 \\
0.25 & 1.7 & 0.19 \\
0.50 & 4.6 & 0.26 \\
1.00 & 10.0 & 0.33 \\
\hline
\end{tabular}

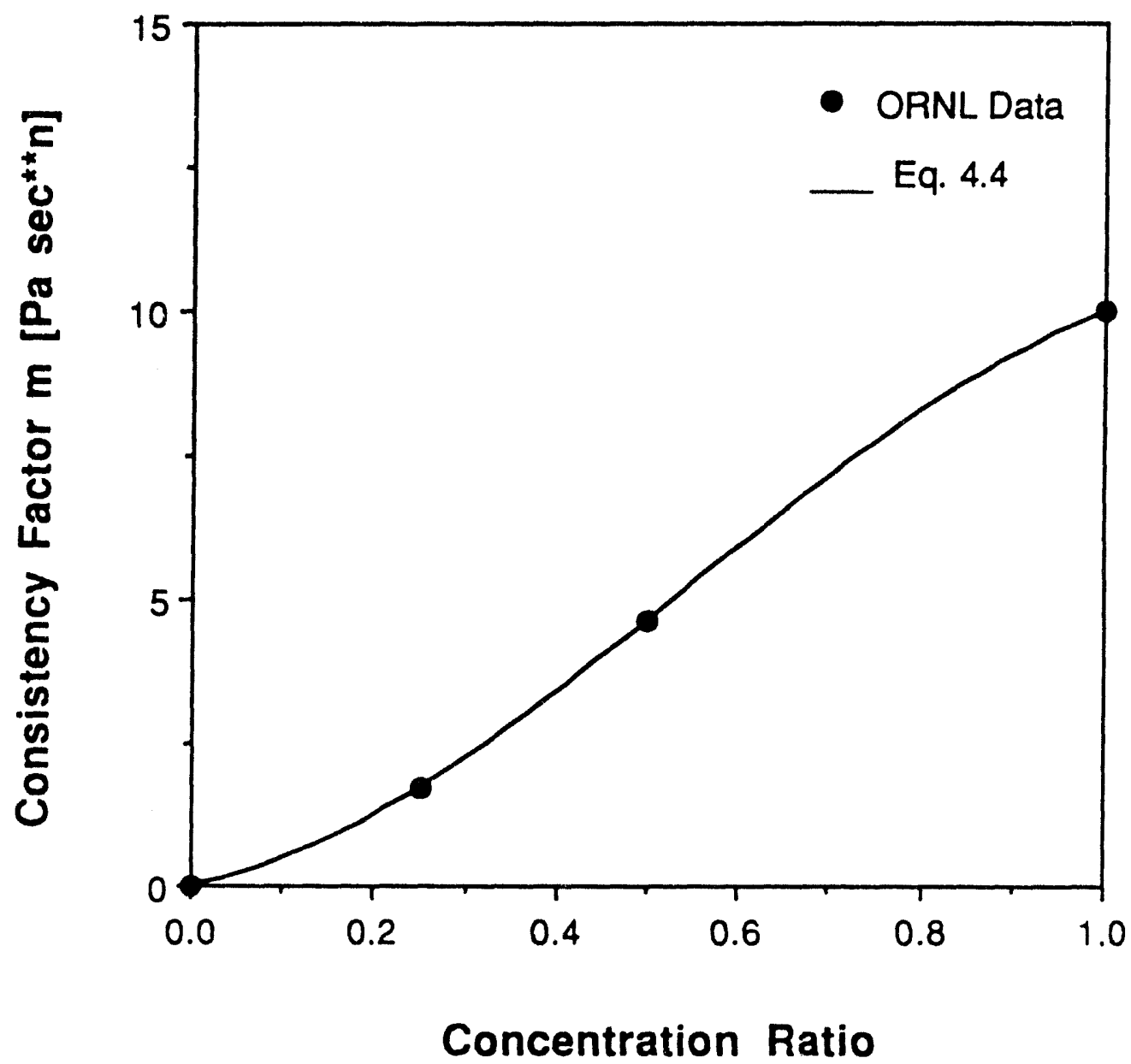

Figure 4.2. Experimental ORNL W-28 Data and Fitted Curve for the Consistency Factor Dependence on Relative Concentration 
The variation of the behavior index $\mathrm{n}$ as a function of $\mathrm{C}_{\mathrm{r}}$ is more complicated than that of the consistency factor $m$. Several approaches were tested to fit the data. In one approach, the following functional form was chosen:

$$
n\left(C_{r}\right)=\alpha\left[e^{-\beta\left(C_{r}-\gamma\right)}-\delta\right]^{2}+\varepsilon
$$

To find the five constants $(\alpha, \beta, \gamma, \delta, \epsilon)$ three additional data points were obtained by interpolation and the minimum of the least squares function was computed

$$
f(\alpha, \beta, \gamma, \delta, \varepsilon)=\sum_{i=1}^{7}\left\{n\left(C_{r i}\right)-\alpha\left[e^{-\beta\left(C_{r i}-\gamma\right)}-\delta\right]^{2}-\varepsilon\right\}
$$

Using a symbol manipulator, a residual of 0.0035 was obtained, which occurs when

$$
\begin{aligned}
& \alpha=0.25071 \\
& \beta=3.92897 \\
& \gamma=0.23991 \\
& \delta=0.77305 \\
& \epsilon=0.20552
\end{aligned}
$$

This curve fit, along with the four experimental data points, is shown in Figure 4.3.

It has been reported that for clay suspensions and magnetite media, the behavior index $\mathbf{n}$ decreases and reaches a minimum (Govier and Aziz 1987). For concentrated suspensions of lignite in methanol, $\mathrm{n}$ increases monotonically with concentration (Darby 1986). This behavior is consistent with the curve in Figure 4.3, constructed from interpolation, where for values of $C_{r}$ less than 0.4 there is a minimum; and for values of $C_{r}$ greater than $0.4, n$ increases monotonically.

An alternative functional form for $n\left(C_{r}\right)$ can be represented by the double exponential function

$$
n\left(C_{r}\right)=a^{-C_{r}}+\left(1-b^{-C_{r}}\right)
$$




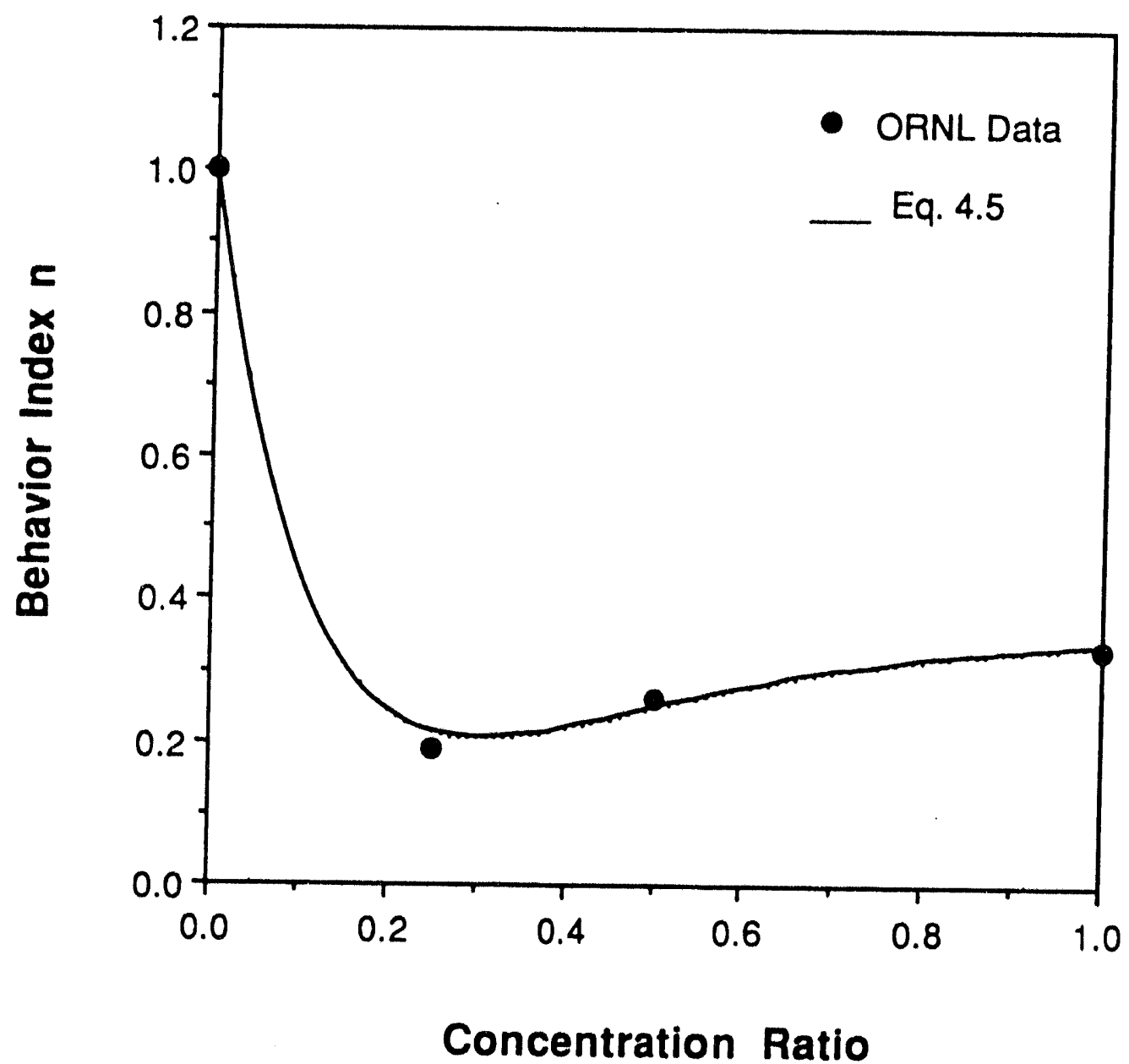

Figure 4.3. Experimental ORNL W-28 Data and Fitted Five Parameter Curve for the Behavior Index Dependence on Relative Concentration

To find the constants $a$ and $b$, the minimum of the least squares function was calculated

$$
g(a, b)=\sum_{i=1}^{4}\left\{n\left(C_{r i}\right)-a^{-C_{r i}}+\left(1-b^{-C_{r i}}\right)\right\}^{2}
$$

which has a residual of 0.0036 for

$$
\begin{aligned}
& a=12510.6 \\
& b=1.55967
\end{aligned}
$$

In this approach, only the four original data points were fit. The curve fit is shown along with the data in Figure 4.4. 


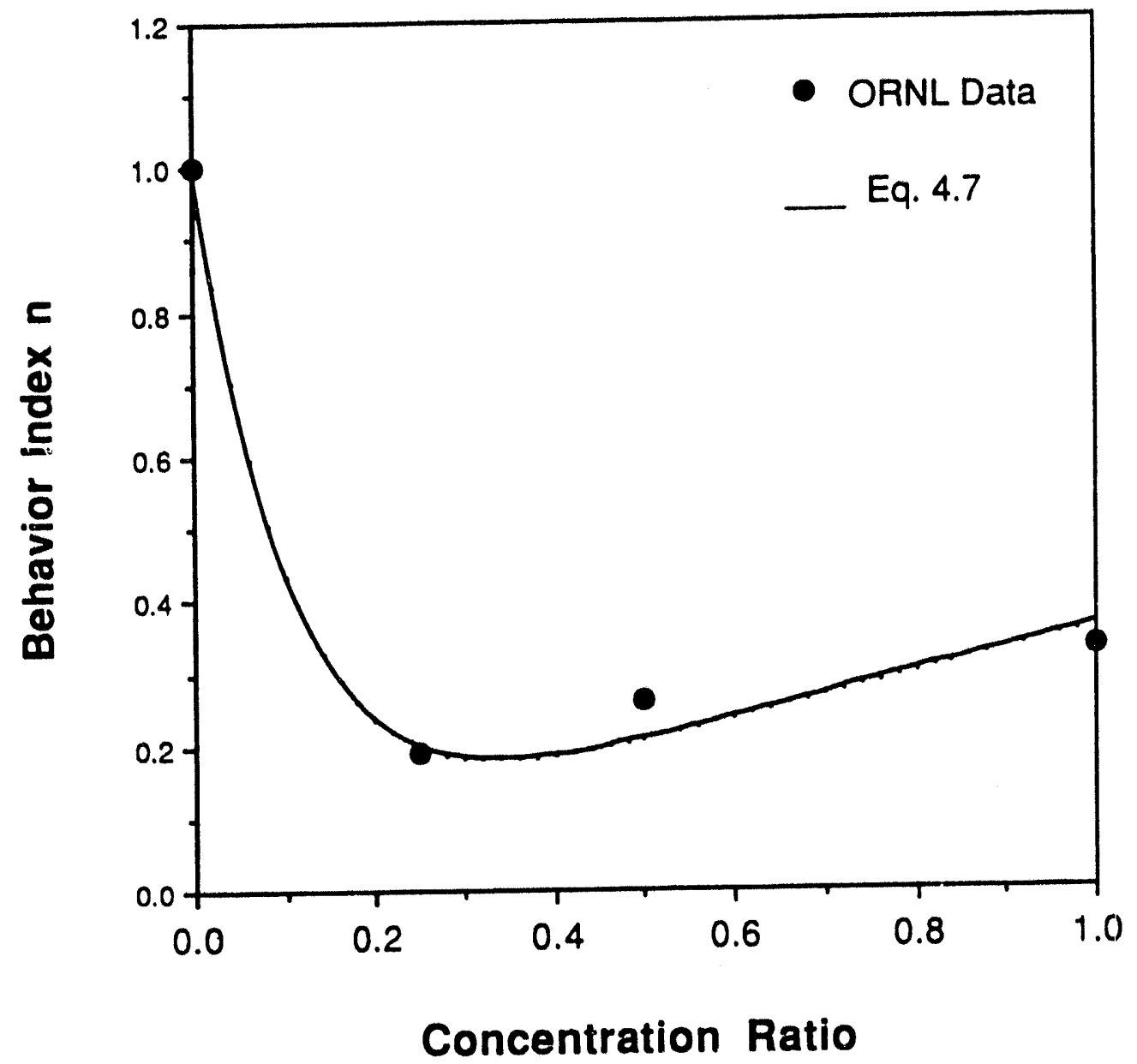

Figure 4.4. Experimental ORNL W-28 Data and Fitted Two Parameter Curve for the Behavior Index Dependence on Relative Concentration

Of these two models for the concentration dependence of the behavior index, the first Equation (4.4) required additional interpolated data points. Furthermore, considering that the data point for $\mathrm{n}$ at $\mathrm{C}_{\mathrm{r}}=1$ is suspect (Youngblood et al. 1991), the second function was chosen for use in the present work because it has a simpler form, it has fewer constants, and it adequately represents the dependency in the data.

An additional quantity required in our simulations is the value of the maximum packing factor $\mathrm{C}_{\mathrm{v}, \mathrm{m}}$. It can be calculated from data for the pure sludge in tank W-28 given by Ceo et al. (1990) by using the relationship 


$$
C_{v, m}=\frac{T_{s}}{1-T_{s}} \frac{\rho_{1}}{\rho_{s}}
$$

where $T_{s}$ is the total fraction of solids by weight, $\rho_{l}$ is the density of the bulk liquid, and $\rho_{\mathrm{S}}$ the density of the undissolved solids. With the reported values:

$$
\begin{aligned}
& \mathrm{T}_{\mathrm{s}}=0.5140 \\
& \rho_{\mathrm{I}}=1.2852 \mathrm{~g} / \mathrm{ml} \\
& \rho_{\mathrm{s}}=2.0000 \mathrm{~g} / \mathrm{ml}
\end{aligned}
$$

it was found that $\mathrm{C}_{\mathrm{v}, \mathrm{m}}=0.68$.

\subsubsection{Implementation in TEMPEST}

To take advantage of the numerical methods utilized in the simulation of flow of Newtonian fluids for the computation of strain-rate-dependent (non-Newtonian) fluids, the rheological equation of state Equation (4.2) must be recast into a Newtonian-like constitutive equation

$$
\tau=\mu_{\text {eff }} \dot{\gamma}
$$

This can be accomplished by expressing an effective or apparent viscosity as

$$
\mu_{\text {eff }}=m\left(C_{r}\right) \dot{\gamma}^{n\left(C_{r}\right)-1}
$$

Computationally, $\mu_{\text {eff }}$ can be updated with the calculation of the strain rate at the previous time step.

An inherent weakness of the power law formulation is that for shear-thinning (pseudoplastic) fluids, in which $n\left(C_{r}\right)<1, \mu_{\text {eff }} \rightarrow \infty$ as $\dot{\gamma} \rightarrow 0$. Because simulations are started from a quiescent state, during the first few time steps one would expect to encounter some regions in the computational domain in which the magnitude of $\dot{\gamma}$ is very small and thus $\mu_{\text {eff }}$ is large. The maximum time step in the numerical solution of the discretized equations of motion has a stability requirement that is proportional to the inverse of viscosity. Numerical procedures in TEMPEST include the option of treating viscosity terms implicitly to reduce the explicit stability limitation. However, this implicit approach can yield inaccurate solutions if the explicit viscosity time step limit is greatly exceeded. In light of this potential problem, it is 
necessary to investigate time step size and stability of calculations which use the power law (pseudoplastic) formulation for the stress-strain relationship.

\subsubsection{Implementation Testing}

The cubic function in $\mathrm{C}_{\mathrm{r}}$ for the consistency factor Equation (4.4) and the double exponential function for the behavior index Equation (4.7) were implemented into TEMPEST. The choice of Equation (4.7) rather than Equation (4.5) for the behavior index is one of convenience, because of its simplicity and reasonable accuracy (additional experimental data would be needed to determine which functional form better describes the rheology of the sludge).

To check the validity of these models, three rheograms for concentration ratios $C_{r}$ of 0.25 , 0.5 , and 1.0 were indirectly computed with TEMPEST. Using a judiciously chosen onedimensional velocity profile for laminar flow of a power-law fluid between parallel moving plates with a constant concentration distribution, a rheogram can be constructed. With reference to Figure 4.5 , for a velocity profile given by

$$
u=\frac{1}{2} z^{2}, \quad v=0, \quad w=0
$$

the only nonzero contribution to the strain-rate is

$$
\dot{\gamma}=\frac{\mathrm{du}}{\mathrm{dz}}=\mathrm{z}
$$

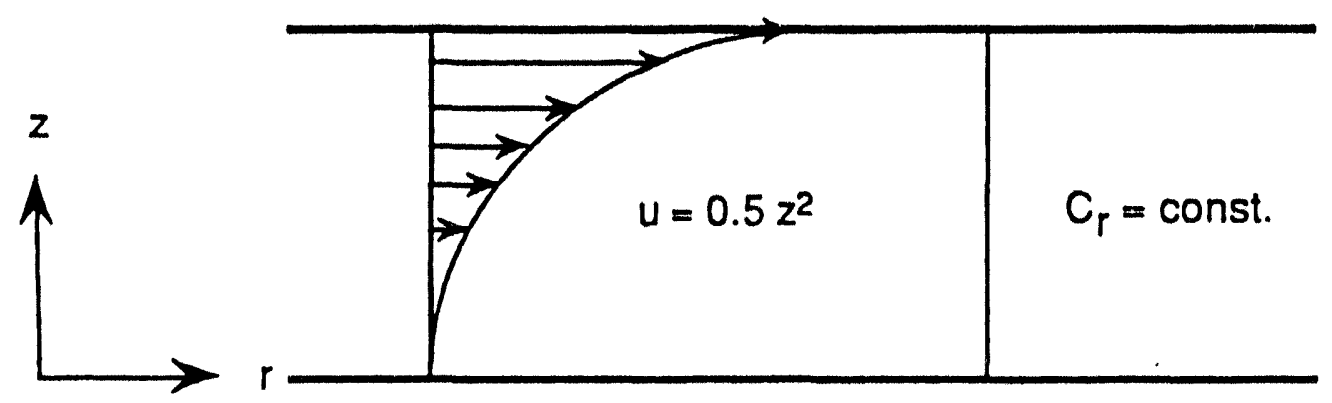

Figure 4.5. Velocity and Concentration Fields Between Parallel Plates Used to Indirectly Construct Rheograms with TEMPEST 
Consequently, there is only one nonzero term of the shear-stress, which can be written as

$$
\tau=2^{(n-1) / 2} \mu_{\text {eff }} z
$$

where $\mu_{\text {eff }}$ is the effective dynamic viscosity computed by TEMPEST. Results of these computations using the power law model for W-28 sludge are displayed in the form of a rheogram in Figure 4.6. Excellent agreement with the experimental data of Ceo et al. (1990) is obtained. This agreement supports the correctness of the model implementation in TEMPEST.

\section{Rheology of MVST W-28 Sludge}

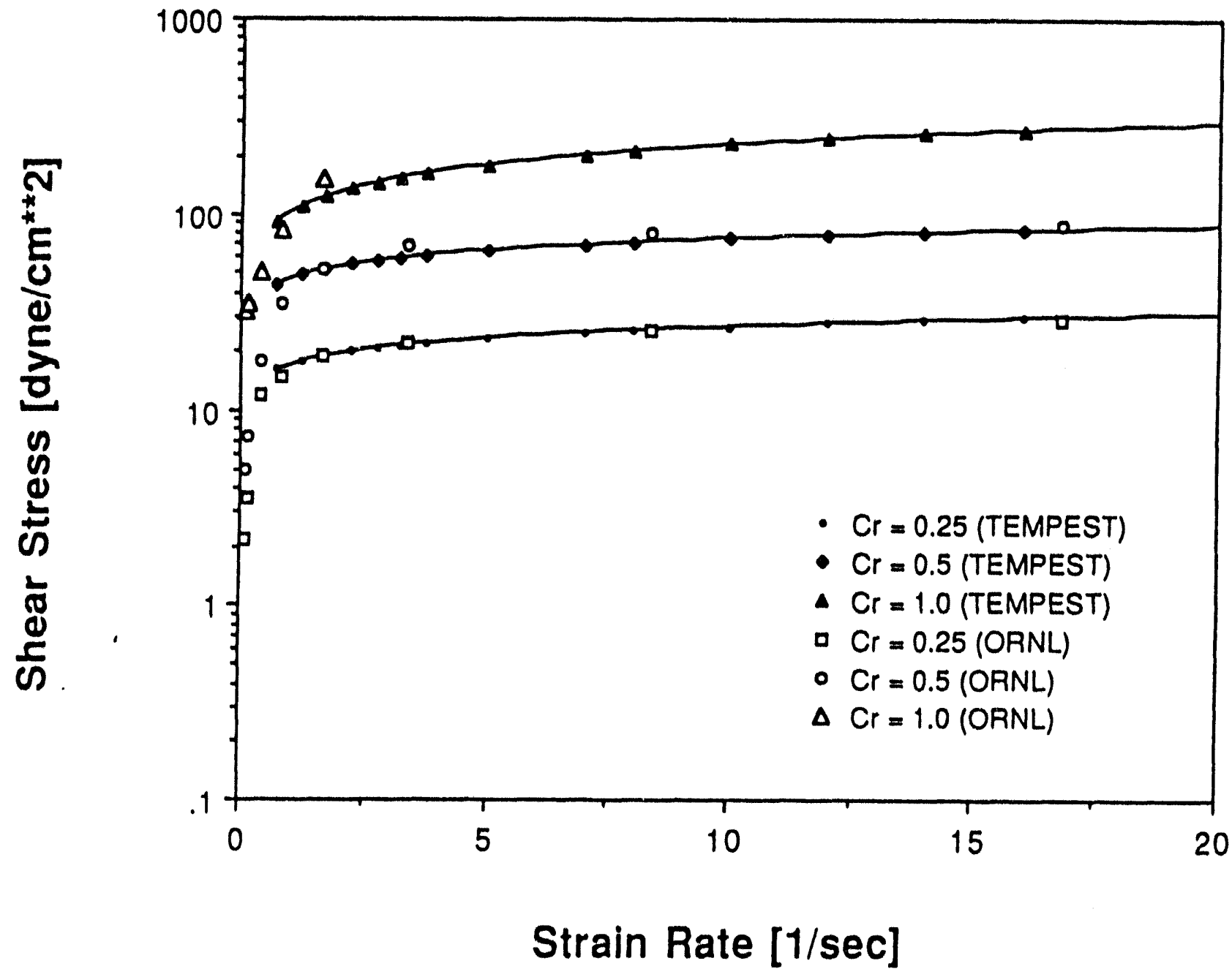

Figure 4.6. Comparison Between Rheological Data from Various Dilution Ratios of W-28 Sludge with Rheograms Numerically Generated by TEMPEST 


\subsection{Time-Step Stability}

TEMPEST is a time-dependent computer program which uses discrete approximations of the governing transport equations. Inherent in the discrete approximation are two explicit numerical stability limitations. One arises from the advection terms and is referred to as the Courant limit. The other arises from the viscous diffusion terms. The latter limitation is potentially very restrictive for high viscosity fluids, such as in the effective viscosity power law formulation of a non-Newtonian fluid. Several calculations are performed to investigate the effect that increasing the time-step viscosity limit has on the stability of solutions. Computations are performed on a submerged, free, axisymmetric jet of a homogeneous nonNewtonian fluid whose rheology is that of the W-28 sludge. That is, the constitutive relation of Equation (4.2) along with the concentration dependent functions [Equations (4.4) and (4.7)] were used. The effect that varying the relative concentration of the sludge has on the viscosity limited time-step and on the CPU time is also examined.

\subsubsection{Mathematical Basis/Numerical Considerations}

Within the TEMPEST solution scheme, there are two time step limiters for stable numerical solutions, $\Delta t_{0}$ and $\Delta t_{m}$, which are based on the cellwise Courant number and the effective momentum diffusivity, respectively. For a cell of "width" $\Delta \mathrm{x}$ in which the velocity of the fluid is $\mathrm{U}$, a measure of the Courant limited time step size is

$$
\Delta t_{c} \propto \frac{\Delta x}{U}
$$

Similarly, a measure of the momentum diffusivity or viscosity limited time step size is

$$
\Delta t_{m} \propto \frac{\rho \Delta x^{2}}{\mu_{\text {eff }}}
$$

To estimate the time required to reach homogenization, numerical simulations must start from a motionless state. During the early stages of the transient there will necessarily be regions within the computational domain in which the strain rate is very small. Thus, in these regions $\mu_{\text {eff }}$ will be large (see Section 4.1.3) and $\Delta \mathrm{t}_{\mathrm{m}}$ (explicit time step) will be small. Consequently, $\Delta \mathrm{t}_{\mathrm{m}}$ will be the predominant time step limiter for stable numesical solutions. Before full homogenization or steady state is computationally achieved, calculations may require considerable amounts of CPU time if $\Delta \mathrm{t}_{\mathrm{m}}$ is utilized. However, TEMPEST has a solution option which can treat the viscous terms implicitly. In that case, the explicit limit $\Delta \mathrm{t}_{\mathrm{m}}$ can be 
$\Delta t_{m}$ can be exceeded. Then the issue is not stability but accuracy of the numerical solutions. Inaccurate solutions could arise in transient calculations in regions where steep gradients are present, e.g., the sludge/supernatant interface.

\subsubsection{Test Problems: Axisymmetric Plug Flow and Free Jet}

The time step size for stable numerical solutions is investigated in an axisymmetric free jet and in a plug flow. The working fluid has the same rheology as the W-28 sludge as represented by Equation (4.2) with functional forms for the consistency factor $m\left(C_{r}\right)$ and the behavior index $n\left(C_{r}\right)$ given by Equations (4.4) and (4.7), respectively. The computational domain is based on the similar dimensions of a typical MVST, i.e., radius of $12 \mathrm{ft}$ and a length of $60 \mathrm{ft}$. The purpose of performing computations in this geometry was to address the following:

1. the effect of concentration dependent non-Newtonian theology on the time step limiters and thus the CPU time,

2. the effect of increasing the viscosity limited time step on the accuracy of numerical solutions.

For each test, the concentration was fixed throughout the computational domain (homogeneous concentration distribution of sludge). The first set of tests was performed by assuming that along the tank's centerline a constant velocity is imposed, which simulates a plug flow across the tank. The second set of tests consisted of computations in which there is a momentum source at the center at one end of the tank. For each set, several velocity fields were computed for various concentration values, ranging from pure supernatant to pure sludge.

Figure 4.7 presents the effect of varying the relative sludge concentration on the average time step size limiter and on the CPU time for simulations, under plug flow conditions. For these runs the viscosity time step limiter is 10 times langer than the explicit stable time step. In Figure 4.7, the CPU times are normalized with the CPU time required to compute the Newtonian case, i.e., when the relative sludge concentration is zero. Note that for relative sludge concentrations less than 0.15 , the time step size is limited by the Courant number, which in this case corresponds to $7.3 \times 10^{-3} \mathrm{sec}$. However, for concentrations greater than about 0.15 , the problem becomes viscosity-time-step limited. Similarly, for concentrations less than 0.15 , the relative CPU time is fairly constant; whereas, outside this range the CPU time increases linearly with concentration. The increase in the required CPU time is linked with the departure of the sludge rheology from that of a Newtonian fluid. This can be seen from Figures 4.2 and 4.3 , where the consistency factor and the behavior index functions 


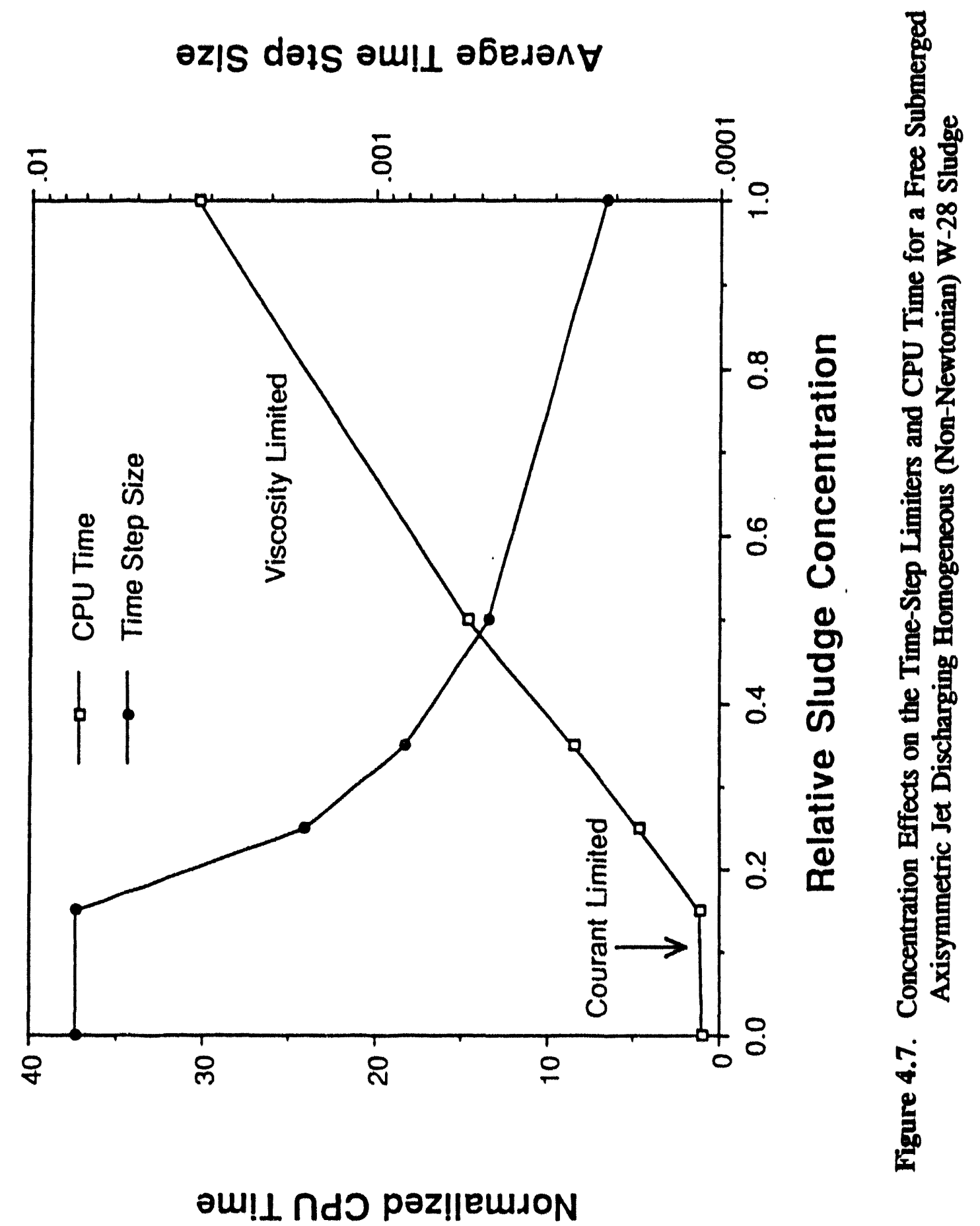


rapidly depart from the Newtonian rheology for concentrations greater than 0.1 . Note that in the case of pure sludge, the CPU time required is 30 times larger than that for the Newtonian case. In a typical full-scale mobilization simulation, the relative concentration within the tank will be between 0.2 and 0.6 , in which case the time-step limiter will be dominated by viscosity. In the present simplified problem, the CPU time needed to compute for this concentration range is 5 to 15 times larger than that required to compute for a Newtonian fluid.

The explicit stable time step for computations in highly viscous fluids (or high effective viscosity) is very small, and thus demands impractical amounts of CPU time. Therefore, the possibility of increasing the limiting time step while maintaining stability and accuracy of the solutions was investigated. This approach is possible when computing with TEMPEST because the terms in the equations of motion where the effective viscosity appears are treated implicitly. In principle, the time step limit could be increased to values as high as the Courant number that limits the convective terms of the equations of motion (which are treated explicitly). The case of maximum sludge concentration required the maximum amount of CPU time (14.8 hr on a Sun Microsystems Sparc II workstation). For this case, the output file was restarted several times with different time step limiters. These limiters were increased up to the Courant number.

Figure 4.8 presents the average time step as a function of the factor (used in the restart input files) by which the viscosity time step limit was multiplied. No oscillations of the velocity field and the pressure were found after $\mathbf{5 0 0}$ time steps of computations from the restart output. Because no numerical instabilities were observed, accuracy was checked by starting the calculation from time zero using the Courant number as time step limiter from the beginning. For this set of runs, computations were carried out using the plug flow and the momentum source configurations. Figure 4.9 (a-d) presents velocity magnitude contours for pure sludge computed with viscosity-limited and Courant-limited time steps. The initial conditions used in Figure $4.9(\mathrm{a}, \mathrm{b})$ correspond to the case of plug flow, whereas for Figure 4.9 (c,d) they correspond to the case of a momentum source. In both cases, the use of the Courant number as the time step limit reduced the CPU time by more than one order of magnitude as compared to the CPU time needed when the time step is limited by viscosity. The agreement between both sets of simulations is excellent, which indicates that it is feasible to increase the implicit viscosity time limit to the Courant limit without sacrificing accuracy or introducing numerical instabilities. 


\section{Stable Time Step Sizes}

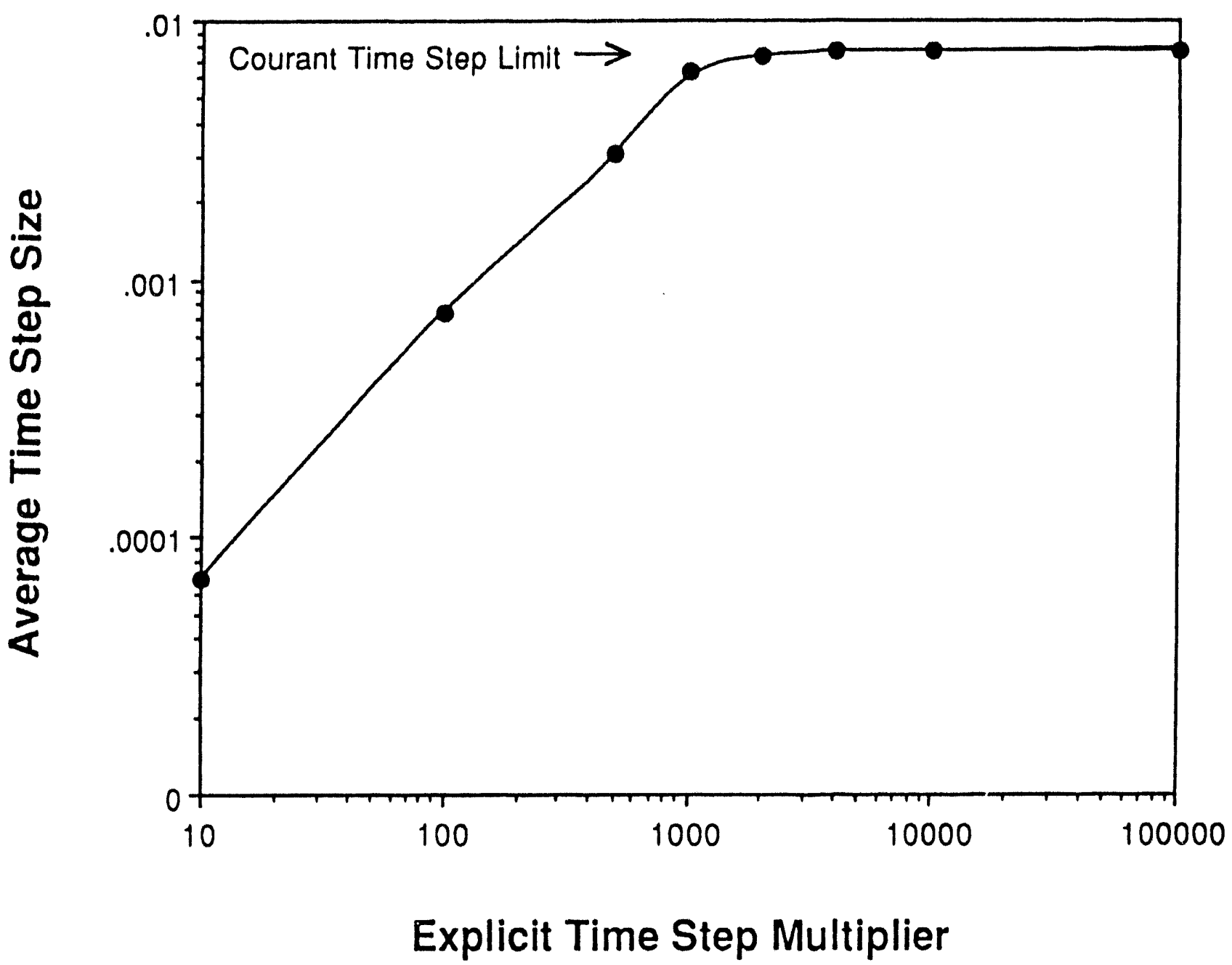

Figure 4.8. Numerically Stable Average Time-Step Size Variation as a Function of the Explicit Time-Step Multiplier for a Free Submerged Axisymmetric Jet Discharging Pure Non-Newtonian Sludge $\left(C_{r}=1\right)$ 

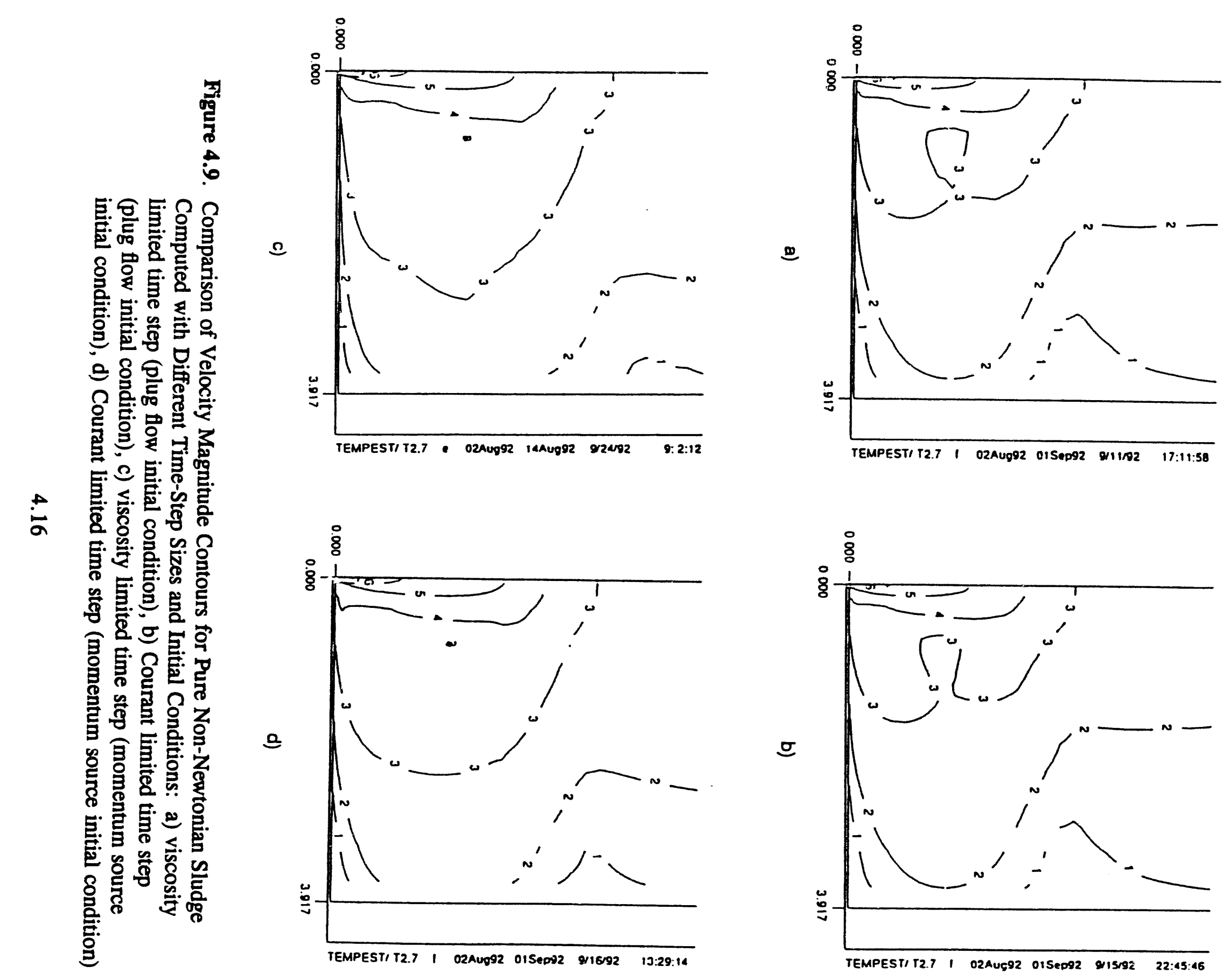
It is concluded from these tests that

- Computations with the non-Newtonian W-28 sludge rheology model are feasible and efficient.

- The pathological characteristic of the power law model at zero strain rate is not prohibitive.

\subsection{Alternative Rheological Approaches}

The ORNL W-28 sludge has been approached as a pseudoplastic (power-law) and as a Bingham plastic (Ceo et al. 1990). The power-law approach implies an infinite effective viscosity at zero strain rate. Its use within TEMPEST for the present problem has been shown not to be unduly restrictive, however. Alternative rheological models have been proposed in the literature to eliminate this power-law restriction at low strain rates. These may be of value in future modeling efforts. Several alternatives are discussed here.

Experimental data for pseudoplastic materials (see Figure 4.10) shows that at very low strain rates (less than $1 \mathrm{sec}^{-1}$ ) the effective viscosity approaches a constant value (zero-strainrate viscosity). At very high strain rates (greater than $10^{4} \mathrm{sec}^{-1}$ ) the effective viscosity approaches asymptotically to a constant value (infinite-strain-rate viscosity). To avoid the appearance of exceedingly high effective viscosities at low strain rates, the following truncated power law model (Bird et al. 1987) could be used:

$$
\mu_{\mathrm{eff}}= \begin{cases}\mu_{0} ; & \dot{\gamma}<\dot{\gamma}_{0} \\ \mu_{0}\left(\frac{\dot{\gamma}}{\dot{\gamma}_{0}}\right)^{\mathrm{n}-1} ; & \dot{\gamma} \geq \dot{\gamma}_{0}\end{cases}
$$

where $\dot{\gamma}_{0}$ is the strain rate below which the effective viscosity is assumed to be constant.

Another model is the four-parameter Carreau equation whose effective viscosities are bounded by two constants $\mu_{0}$ and $\mu_{\infty}$

$$
\mu_{\mathrm{eff}}=\mu_{\infty}+\frac{\mu_{0}-\mu_{\infty}}{\left[1+\left(\lambda_{\dot{\gamma}}\right)^{2}\right]^{1-\frac{\mathrm{n}}{2}}}
$$




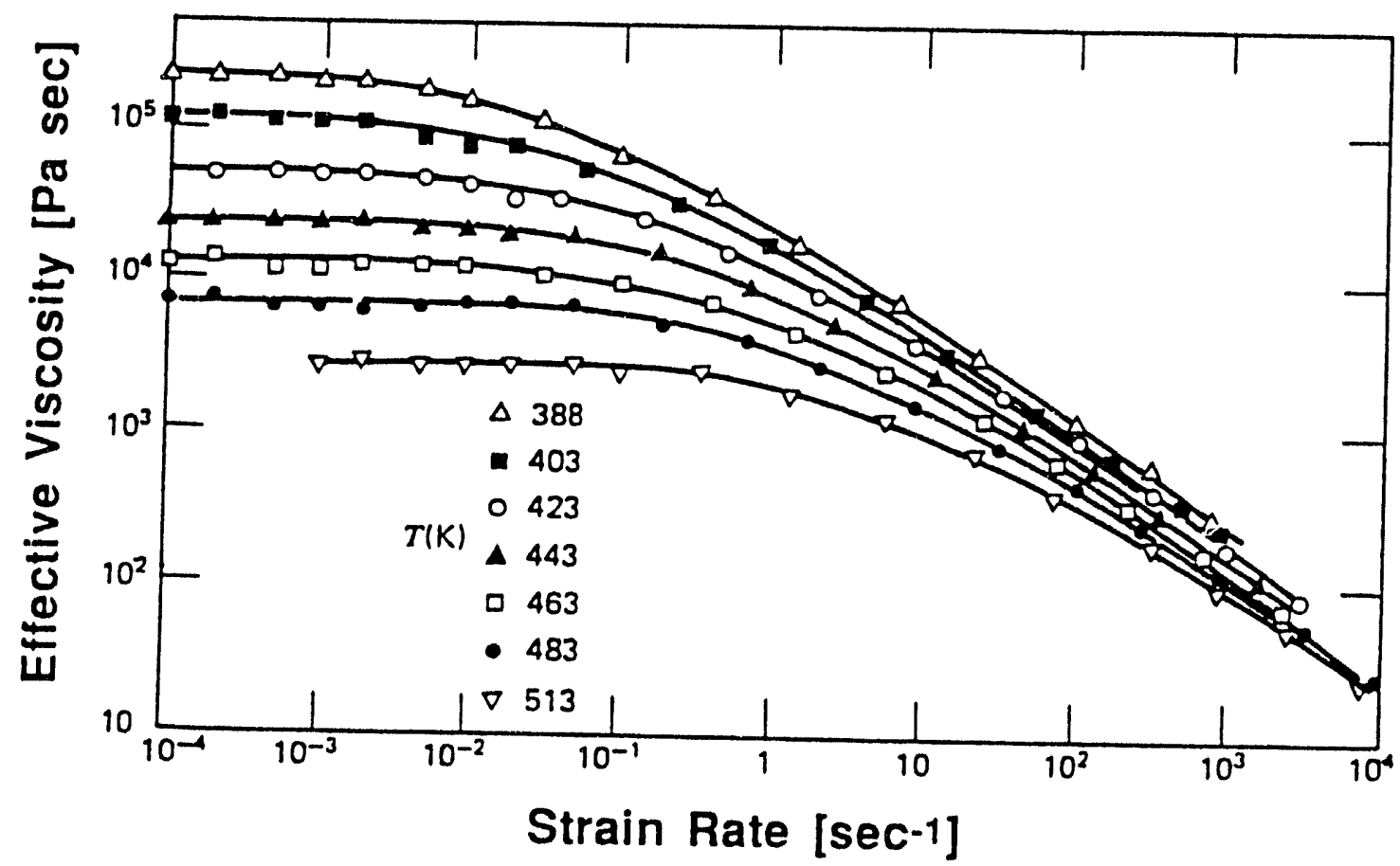

Figure 4.10. Experimental Data of Effective Viscosity as a Function of Strain Rate for a Pseudoplastic Fluid (polyethylene melt, Bird et al. 1987)

where $\mu_{\mathrm{o}}$ is the zero-strain-rate viscosity, $\mu_{\infty}$ is the infinite-strain-rate viscosity, $\lambda$ is a time constant, and $\mathrm{n}$ is the behavior index. This model more closely represents the rheological characteristics shown in Figure 4.10.

For materials with high apparent yield stress, the Herschel-Bulkley model fits experimental data better than the popular Bingham model. The Herschel-Bulkley model is expressed mathematically as:

$$
\begin{aligned}
& \dot{\gamma}=0 ; \quad \tau<\tau_{0} \\
& \mu_{\text {eff }}=\mathrm{m}_{\dot{\gamma}} \dot{\mathrm{n}}^{-1}+\frac{\tau_{\mathrm{o}}}{\dot{\gamma}} ; \quad \tau \geq \tau_{\mathrm{o}}
\end{aligned}
$$

Both the Bingham model and a form of the Herschel-Bulkley model are implemented in TEMPEST. Additional testing, beyond the scope of the present work, is required to determine if any of these models provide a better representation of a wider range of ORNL sludges. 


\subsection{Sludge Mixing Simulations in a Half-Filled 1/6-Scale Tank}

Subsequent to the preliminary investigations of numerical stability, computational efficiency, and rheological modeling approaches to ORNL W-28 sludge, calculations were conducted to investigate mobilization and mixing in a 1/6-scale tank representative of the geometry of the horizontal MVSTs. These calculations were preliminary to simulation of sludge mobilization experiments in scaled tanks to be conducted at ORNL. Figure 5.1 presents a schematic of the computational geometry representative of the full size MVSTs. The 1/6-scale version of this geometry has a length of $10 \mathrm{ft}$ and a diameter of $2 \mathrm{ft}$. The 1/6-scale experiment model at ORNL is constructed of clear plastic to aid in visualization of mixing experiments. The MVST configuration allows access only at points $1 / 4$ and 1/2 of the distance along tank length. Sludge layers are expected to range from 15 to $50 \%$ of the tank diameter, with varying depths of supernatant above the sludge layer.

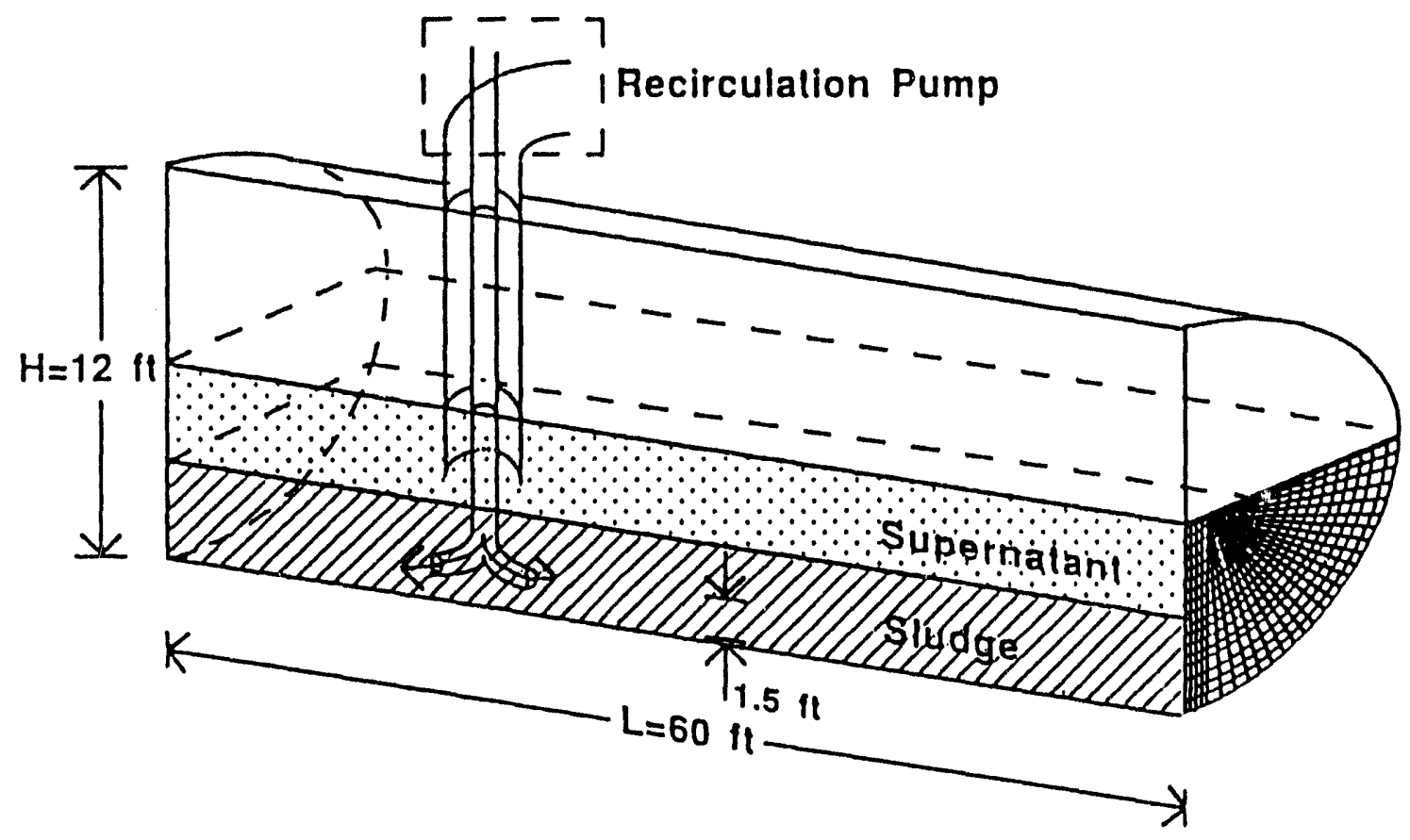

Figure 5.1. Schematic Representation of the Computational Domain for the Melton Valley Storage Tanks 
The present work is preliminary in nature and focuses on investigating the effect of jet velocity on the mobilization and mixing. The complete parameter space for mixing investigations would necessarily have to cover a wide range of parameters. These include:

- Supernatant parameters (rheology, layer depth)

- Sludge parameters (rheology, layer depth, particle characterization)

- Mixing jet parameters (jet velocity, jet diameter, axial location in the tank, height above floor, recirculating pump intake location).

To completely investigate the parameter space to define mobilization and homogenization time would require extensive numerical simulations. Such an extensive investigation can only be proposed and completed subsequent to confirmation of the preliminary calculations in comparison to experimental data in the scaled tanks. Such experiments are planned at ORNL.

\subsection{Description of Models}

The computational domain for mixing simulations in the half-filled $1 / 6$-scale geometry is represented by schematics presented in Figures 5.1 and 5.2. Two computational models were assembled - a "finely" resolved one which contained 33,124 cells and a "coarse" one which contained 7,500 cells. The coarse model was used exclusively for results presented herein. TEMPEST generalized coordinates capabilities were used in the model to retain good representation of the tank wall curvature and jet spread. The end view computational grid structure is included schematically in Figure 5.1.

Preliminary calculations of mixing were conducted with 1-in. diameter jets, centrally located at a distance of $2.5 \mathrm{ft}$ from one end of the tank. They were issuing in the centerplane along the direction of the tank axis. The jet centerline was 2 in. above the floor of the tank. A recirculating flow was included in the model, with an intake located vertically above the jets, near the liquid surface. The pump and pump stem were modeled with zero thickness plates and a momentum source to define jet velocity. A jet velocity of $1 \mathrm{ft} / \mathrm{sec}$ was used in preliminary tests.

The definition of jet mixing time in tanks has customarily been defined in qualitative terms as the time lapse in which measurements (electronic or visual detection) reveal no change in concentration in a chosen sampling region. For computational purposes, a mixing time can be 


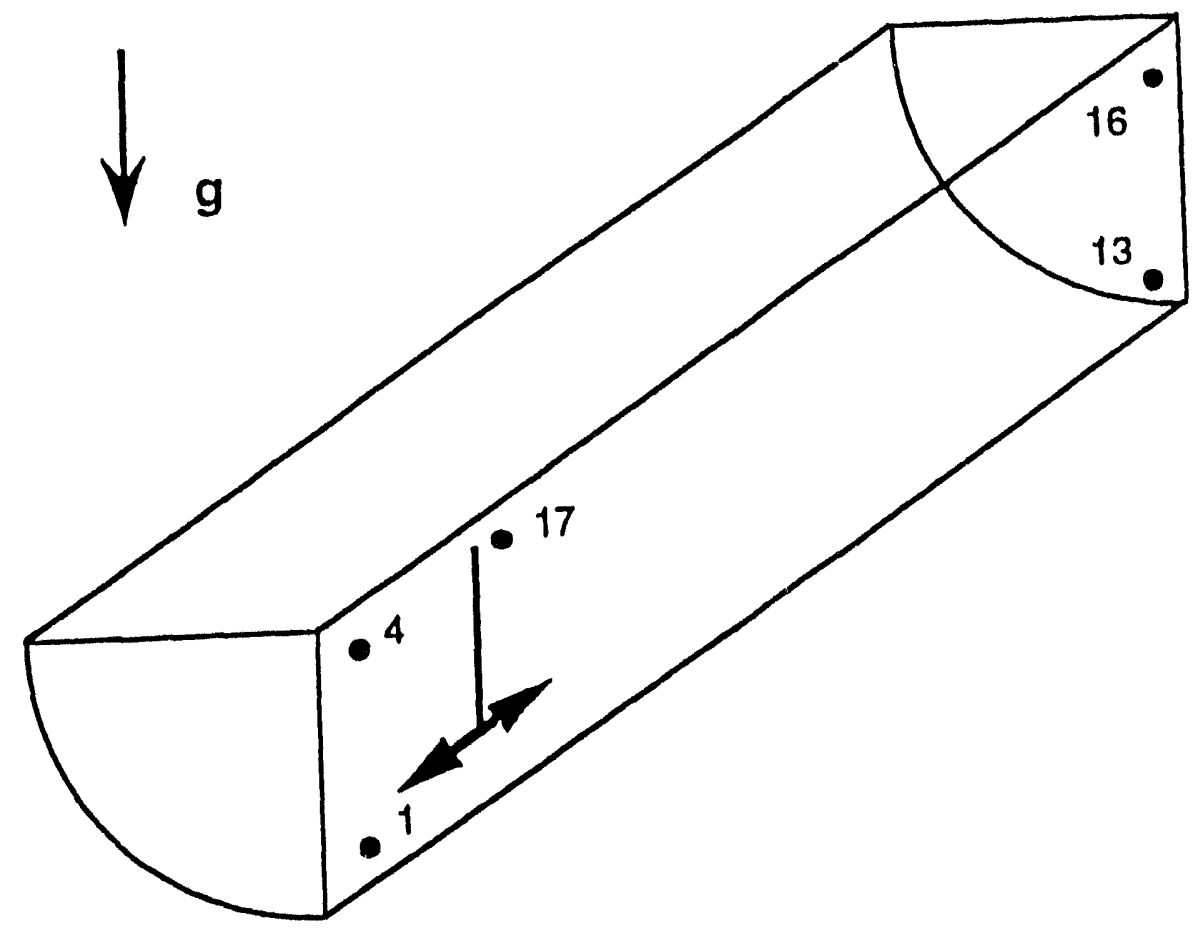

Figure 5.2. Schematic of the Sampling Point Locations from Which Concentration Variations in Time were Monitored to Determine Mixing Times

defined as the time it takes for the extremes of the concentration distribution within the tank to vary by less than some percentage difference about the mean fully mixed concentration. Mixing time can be determined by monitoring the concentration as a function of time at discrete points in the computational domain. To augment this approach, the concentration at the intake to the (modeled) recirculating pump can also be monitored. Points monitored in the calculations are near the ends of the tank and are shown schematically in Figure 5.2. The numbers adjacent to the geometric extremes $(1,4,13$, and 16) and the recirculation line (17) correspond to monitor cell locations in TEMPEST calculations. These monitor cell locations were used to determine the characteristics of mixing and the mixing times for highly viscous Newtonian and non-Newtonian (W-28) sludge in a half-filled 1/6-scale tank.

\subsection{High Viscosity Newtonian Sludge Mixing Simulations}

Calculation results of mixing a Newtonian sludge in a half-filled 1/6-scale geometry are presented. These are preliminary investigations into estimating required times to homogenize or mix the sludge layer. For these calculations, water properties were used for the liquid layer. An exponential concentration-dependent viscosity function was used. For the simulated sludge in these calculations, viscosities ranged up to $10^{4}$ times the base fluid viscosity. 
Independent tests were also conducted to investigate sludge density and molecular and turbulent diffusivity effects on the computations. The effects of diffusivity are investigated because in mixing processes, materials can be transported by both convection and diffusion. The latter is especially important in turbulent mixing, but the former is also important in some mixing systems.

\subsubsection{Exponential Shape Viscosity Model}

Several calculations were conducted in which the density of the sludge and the viscosity of the liquid/solid mixture were varied. Clear water was used as the base fluid. Concentration dependent viscosity was assumed to vary according to an exponential shape curve of the form

$$
\frac{\mu}{\mu_{0}}=A^{C_{r}}
$$

where $\mu_{\mathrm{o}}=$ the clear water viscosity

$$
\mathrm{C}_{\mathrm{r}}=\text { the ratio of the volumetric concentration to the maximum concentration }
$$
$\left(\mathrm{C}_{\mathrm{r}} / \mathrm{C}_{\mathrm{r}}, \mathrm{m}\right)$, and

$$
A=\text { the maximum viscosity ratio at } C_{r}=1 \text {. }
$$

This form is convenient for parameter testing in that the constant A represents a maximum viscosity ratio at $\mathrm{C}_{\mathrm{r}}=1$, the maximum packed concentration of solids in the sludge layer. The maximum packing factor for the solids was $\mathrm{C}_{\mathrm{v}, \mathrm{m}}=0.68$. Values of $\mathrm{A}$ from 1 (pure liquid) to $10^{4}$ were used in the simulations to investigate viscosity effects of the sludge layer. Density effects were investigated by varying the solids specific gravity from 1 to 2 . At a specific gravity of 1 , the solids in the sludge layer represent a neutrally buoyant passive scalar. This neutrally buoyant case in which $A=1$ provides a reference base case. Table 5.1 presents a list of the cases and the primary parameters used in each case.

The laminar and turbulent Schmidt numbers represent the ratio of diffusivity of momentum to the diffusivity of mass (species) for molecular and turbulent diffusion, respectively. These parameters (especially the laminar diffusivity) become important in transport simulations of species with high effective viscosity. In these situations, selection of a Schmidt number on the order of unity means that species mass may diffuse abnormally fast as a result of the high (molecular) viscosity which occurs at viscosity ratios of $10^{4}$. This is not a real physical phenomenon, so selecting a Schmidt number of $10^{9}$ effectively eliminates diffusion (other than numerical). 
Table 5.1. Mixing Simulation Parameters for an Exponential Shape Viscosity Model in a Half-Filled $1 / 6-$ Scale Tank

\begin{tabular}{||c|l|c|c|c|c|}
\hline $\begin{array}{c}\text { Case } \\
\text { Number }\end{array}$ & \multicolumn{1}{|c|}{ Case Iden } & $\begin{array}{c}\text { Solids } \\
\text { Specific } \\
\text { Gravity }\end{array}$ & $\begin{array}{c}\text { Maximum } \\
\text { Viscosity } \\
\text { Ratio (A) }\end{array}$ & $\begin{array}{c}\text { Laminar } \\
\text { Schmidt } \\
\text { Number }\end{array}$ & $\begin{array}{c}\text { Turbulent } \\
\text { Schmidt } \\
\text { Number }\end{array}$ \\
\hline 1 & water & NA & NA & NA & NA \\
1a & sg 1.0 & 1.0 & 1.0 & 0.71 & 0.71 \\
1b & sg 1.5 & 1.5 & 1.0 & 0.71 & 0.71 \\
1c & sg 2.0 & 2.0 & 1.0 & 0.71 & 0.71 \\
2a & mu 1+1 & 2.0 & 10 & 0.71 & 0.71 \\
2b & mu 1+2 & 2.0 & $10^{2}$ & 0.71 & 0.71 \\
2c & mu 1+3 & 2.0 & $10^{3}$ & 0.71 & 0.71 \\
2d & mu 1+4 & 2.0 & $10^{4}$ & 0.71 & 0.71 \\
3b & mu 1+2 Sc0 & 2.0 & $10^{2}$ & $10^{9}$ & $10^{9}$ \\
3c & mu 1+3 Sc0 & 2.0 & $10^{3}$ & $10^{9}$ & $10^{9}$ \\
3d & mu 1+4 Sc0 & 2.0 & $10^{4}$ & $10^{9}$ & $10^{9}$ \\
4d & mu 1+4 Tu0 & 2.0 & $10^{4}$ & $10^{9}$ & 0.71 \\
\hline
\end{tabular}

In the cases where a sludge layer was present, an initial solids species distribution was specified such that it represented a maximum packed $\left(C_{v, m}=0.68\right)$ layer occupying approximately 0.14 of the tank diameter. This amount of solids results in a total solids volume of $3 \mathrm{ft}^{3}$ and a fully mixed volume fraction of solids of 0.366 .

Cases 1 to $1 c$ were computed to investigate the effect of density on mixing times. For these cases, there is a potential for buoyancy effects in the jet flow during the mixing time to cause to lift the jet off the floor as a forced plume. Such a phenomenon has the potential to increase the time it takes to mix the tank contents, relative to the case of pure water.

Cases $2 \mathrm{a}$ to $2 \mathrm{~d}$ were computed to investigate the effect of viscosity on mixing times. These cases yielded the spurious result that as the maximum viscosity increased, the mixing time decreased. This effect was determined to be caused by the artifact discussed above, viz., an abnormally high laminar (molecular) diffusivity being calculated for the high effective viscosity cases. 
Cases $3 \mathrm{~b}$ to $3 \mathrm{~d}$ were computed to further investigate mixing times for the arguably conservative situation where both laminar (molecular) and turbulent diffusivity of the solid species were negligible. For this situation, solids are mixed according to the developing flow patterns of the jet induced circulations in the tank. The last case computed, Case $4 d$, was a numerical experiment to isolate the computed significance of turbulent diffusivity relative to that of molecular diffusivity. Characteristic mixing times are examined in these numerical experiments through the use of time-dependent concentration curves.

Time dependent concentration results are presented in the form of volume fraction of solids at five locations in the computational domain. The locations are shown schematically in Figure 5.2. The numbers 1, 4, 13, and 16 are monitor locations in the extreme comer cells of the computational domain, in the vertical centerplane. Two of these locations (1 and 13) are initially in the sludge layer and adjacent to the floor of the tank. The other two (4 and 16) are initially in the supernatant and adjacent to the supernatant surface. Number 17 is the monitor location at the intake of the recirculating purpp model in the computational domain.

Case 1 (pure water) has no indicator of species volume fraction and represents a base case to investigate the nature of the base fluid mixing and jet development. The results of cases 1a to $1 \mathrm{c}$ in which specific density of the solids is the primary parameter are presented in Figure 5.3 to 5.5 , respectively. Case 1a computes a situation in which there is no density effect, because the solid has a speciffc gravity of 1 and thus acts a truly passive scalar. Note that for this case, there is very rapid total mixing, with the average concentration of 0.3658 being reached within 2 minutes. For case $1 \mathrm{~b}$ with solids speciffc gravity of 1.5 , the time to reach a uniformly mixed state is nearly 5 minutes. For the heaviest solids simulated, with a specific gravity of 2 , the time to reach a mixed state is nearly 6 minutes. These results support the contention that density effects in the jet flow play a role in tank mixing. This contention has been made (Bamberger et al. 1991) in 1/12-scale modeling analysis. It has also been borne out in other tank mixing analysis (Bamberger et al. 1993). Figure 5.6 presents a concatenation of the time variation of the solids concentration at the pump intake (monitor location 17). In this figure, the variation of the mixing time as a function of solids specific density is quite evident.

Results for the time dependent variation of solids concentration with variation of the viscosity ratio are presented in Figure 5.7. The results included in the figure are for solids with a specific density of 2 , and include Cases $1 \mathrm{c}$ and $2 \mathrm{a}$ to $2 \mathrm{~d}$. The viscosity ratio varies from 1 to $10^{4}$. It is interesting to note that the time to homogenize the mixture is relatively independent of viscosity until the viscosity ratio exceeds $10^{3}$. What is particularly interesting about these results is that the time to homogenize the mixture decreases with increasing viscosity. This result is counter intuitive and is probably not a real trend. It results directly from the molecular diffusivity of the solids being proportional to the effective viscosity and the 


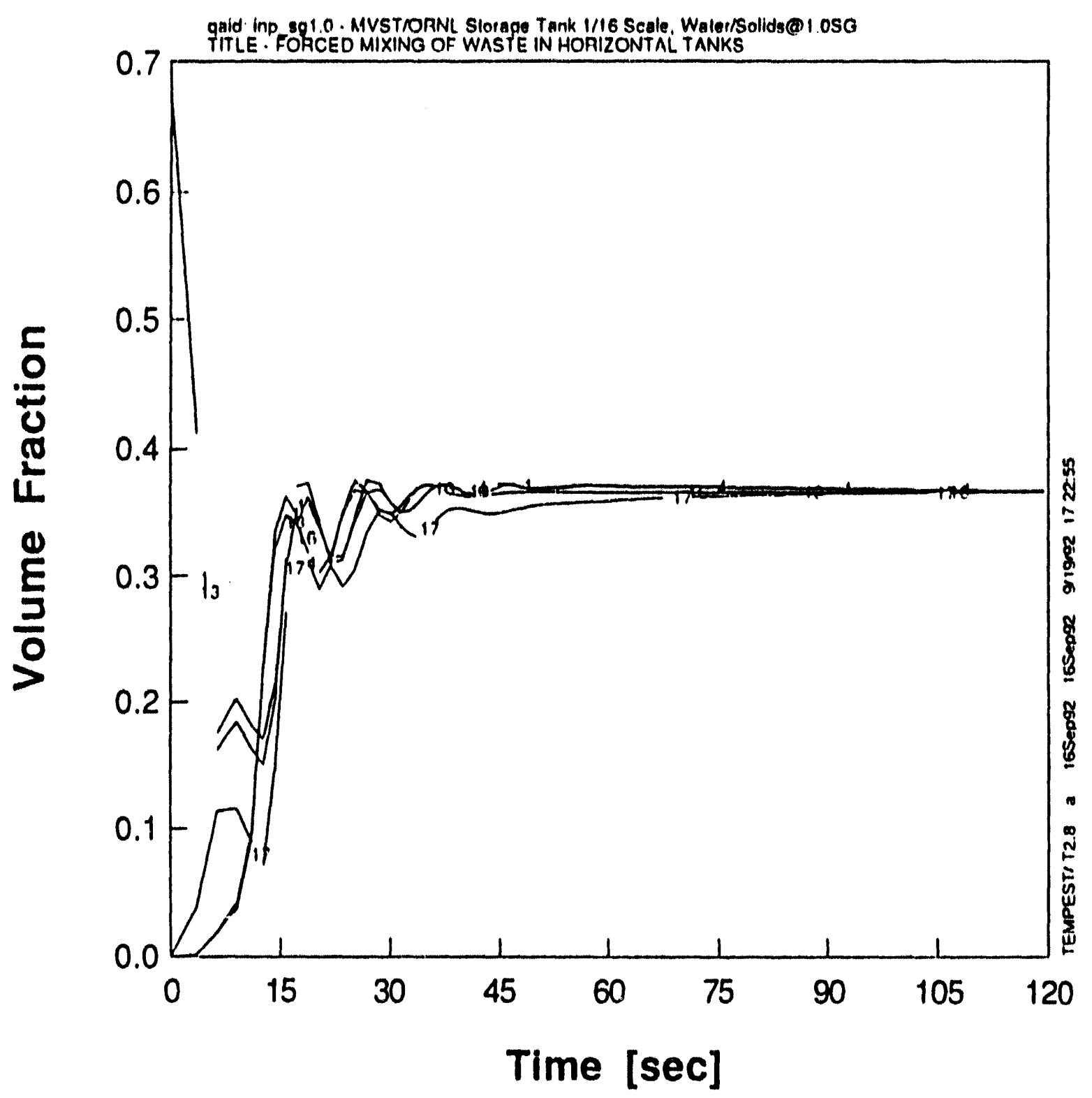

Figure 5.3. Time Dependence of Solids Concentration for Neutrally Buoyant Particles in Water Without Concentration Dependent Viscosity (Case 1a, SG =1) 


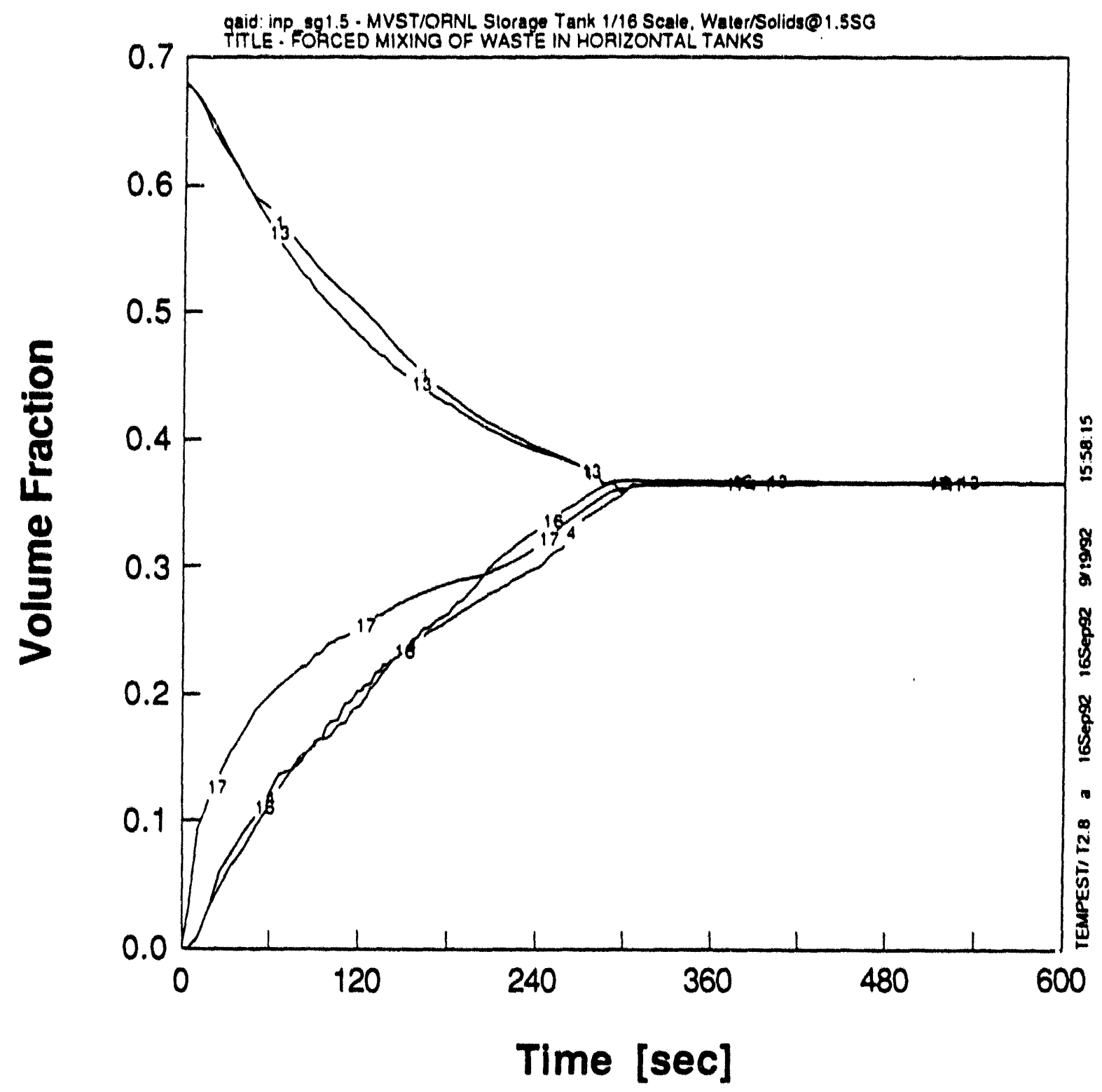

Figure 5.4. Time Dependence of Solids Concentration for Neutrally Buoyant Particles in Water Without Concentration Dependent Viscosity (Case Ib, SG $=1.5$ ) 


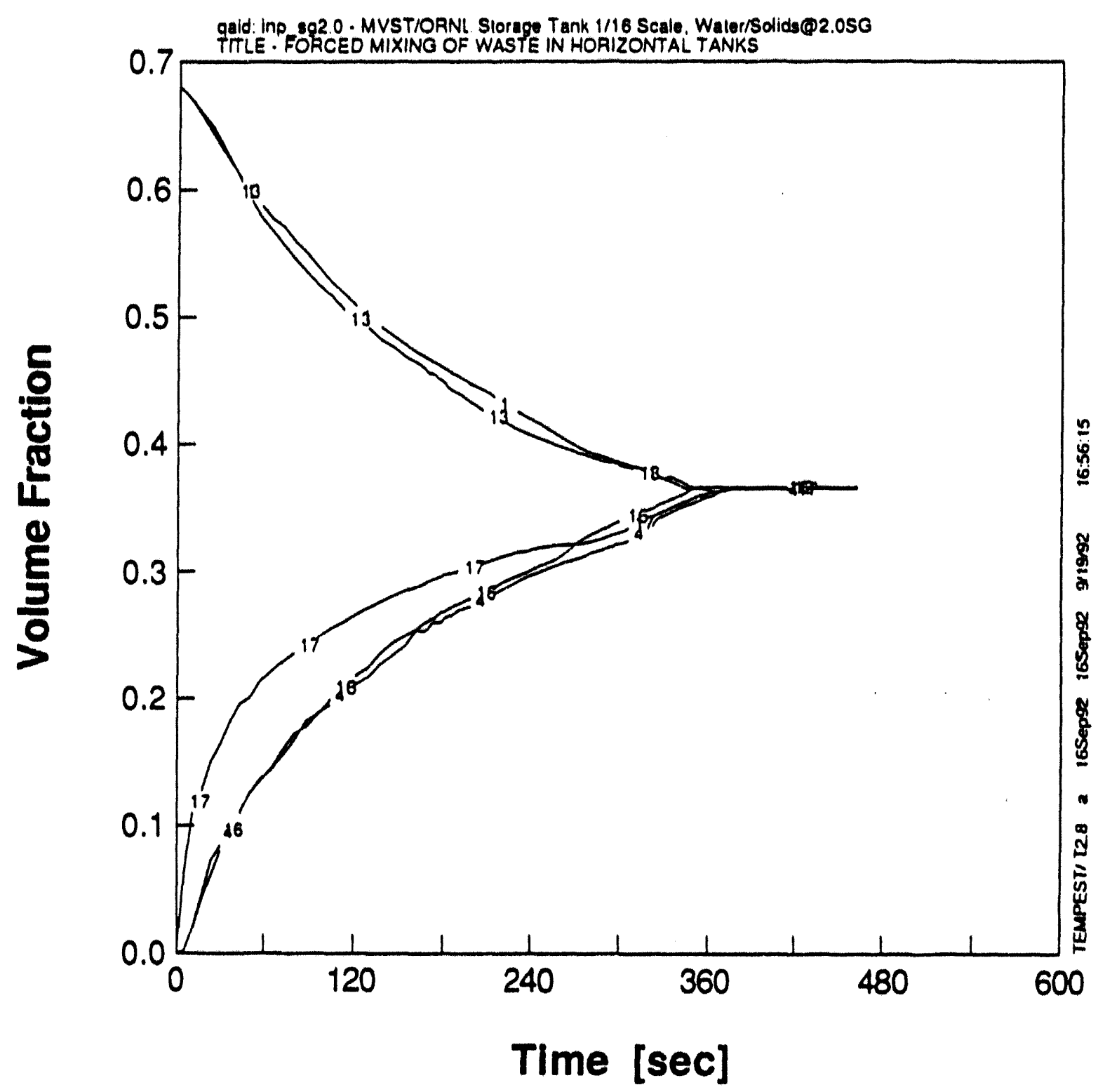

Figure 5.5. Time Dependence of Solids Concentration for Neutrally Buoyant Particles in Water Without Concentration Dependent Viscosity (Case 1c, $S G=2$ ) 


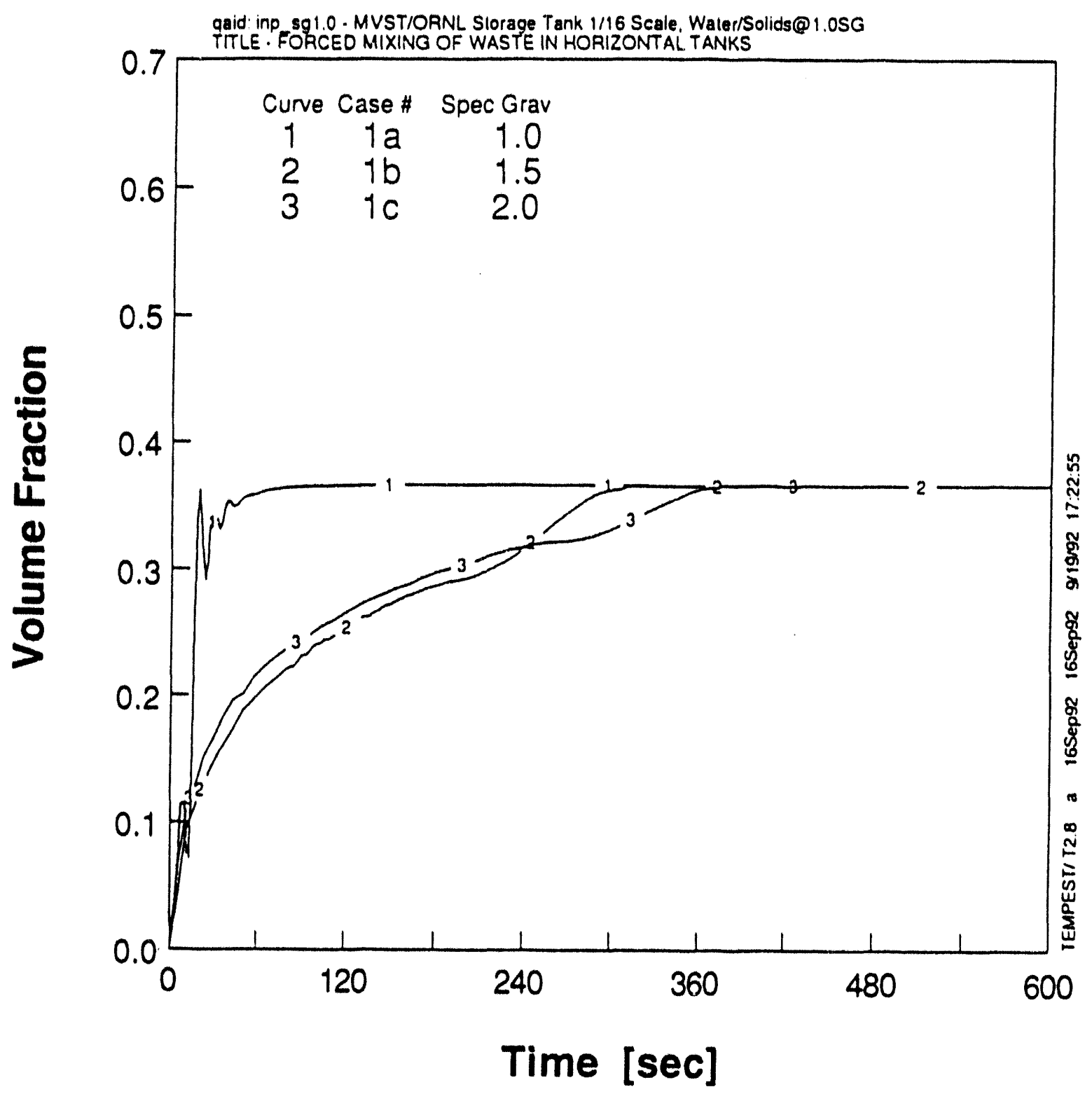

Figure 5.6. Composite of the Time Variation of the Solids Concentration at the Modeled Recirculating Pump Intake Showing the Effect of Solids Density on Mixing Time (Cases 1a-1c) 


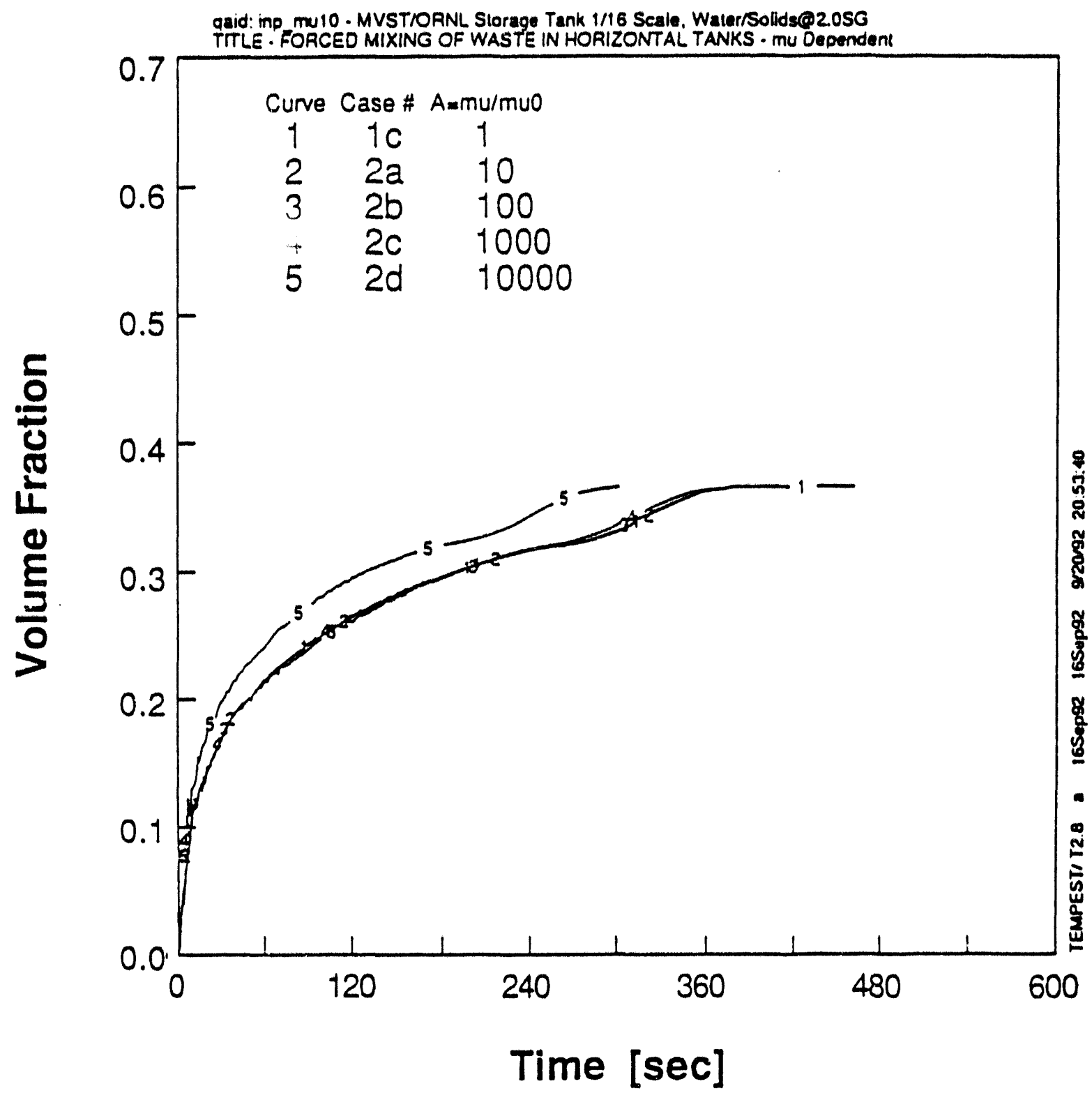

Figure 5.7. Composite of the Time Variation of the Solids Concentration at the Modeled Recirculating Pump Intake Showing the Effect of Maximum Viscosity Ratio for High Molecular and Turbulent Diffusivity 
Schmidt number in the computer model. As the maximum viscosity ratio increases, the molecular diffusivity greatly exceeds the turbulent mixing due to the jet, and as a result, the material (artificially) diffuses too fast. This set of results is presented here because it points to a caution that must be exercised in modeling concentration-dependent viscous sludges.

Figures 5.8 to 5.10 present the time dependent results for Cases $3 \mathrm{~b}$ to $3 \mathrm{~d}$, respectively, for solids concentration at five monitor locations, four at the extremes of the geometry and one (number 17) at the modeled recirculating pump intake. The most notable differences in these results are in the time dependent variation of the solids adjacent to the floor at the ends of the tanks (curves 1 and 13). The largest maximum viscosity ratio cases indicate an expected result that the material adjacent to the floor takes longer to mobilize. Figure 5.11 presents similar results for Case $4 \mathrm{~d}$ in which the turbulent Schmidt number is 0.71 (effectively active turbulent diffusivity of solids) while the molecular Schmidt number is $10^{9}$ (effectively inactive molecular diffusivity). Comparison of Figures 5.10 and 5.11, which are for the same maximum viscosity ratio of $10^{4}$, shows a quite marked difference. This indicates that the turbulent diffusivity is very dominant in the overall mixing process as time proceeds.

A composite of the modeled recirculating pump intake results is presented in Figure 5.12 for Cases $1 c, 3 b$ to $3 d$, and $4 d$. For a consistent set of diffusivity assumptions (Cases $3 b$ to $3 d)$, these results show a significant increase in mixing time with increase in maximum viscosity ratio. These results further indicated that assumed diffusivity can greatly affect predicted mixing times (compare Case $4 \mathrm{~d}$ to $3 \mathrm{~d}$ ).

\subsubsection{Power Shape Viscosity Model}

In the previous section, preliminary investigations computed with an exponential shape (Newtonian) concentration-dependent viscosity function were presented. In this section, an additional investigation using a power shape (Newtonian) concentration-dependent viscosity function is presented. This model is then used to compute mixing times in the 1/6-scale tank.

The basis for the model is as follows.

For strain rates greater than $1 \mathrm{sec}^{-1}$, the W-28 sludge power law model of Equations (4.4) and (4.7) is in excellent agreement with data (see Figure 4.1). At $\dot{\gamma}=1 \mathrm{sec}^{-1}$, the effective 


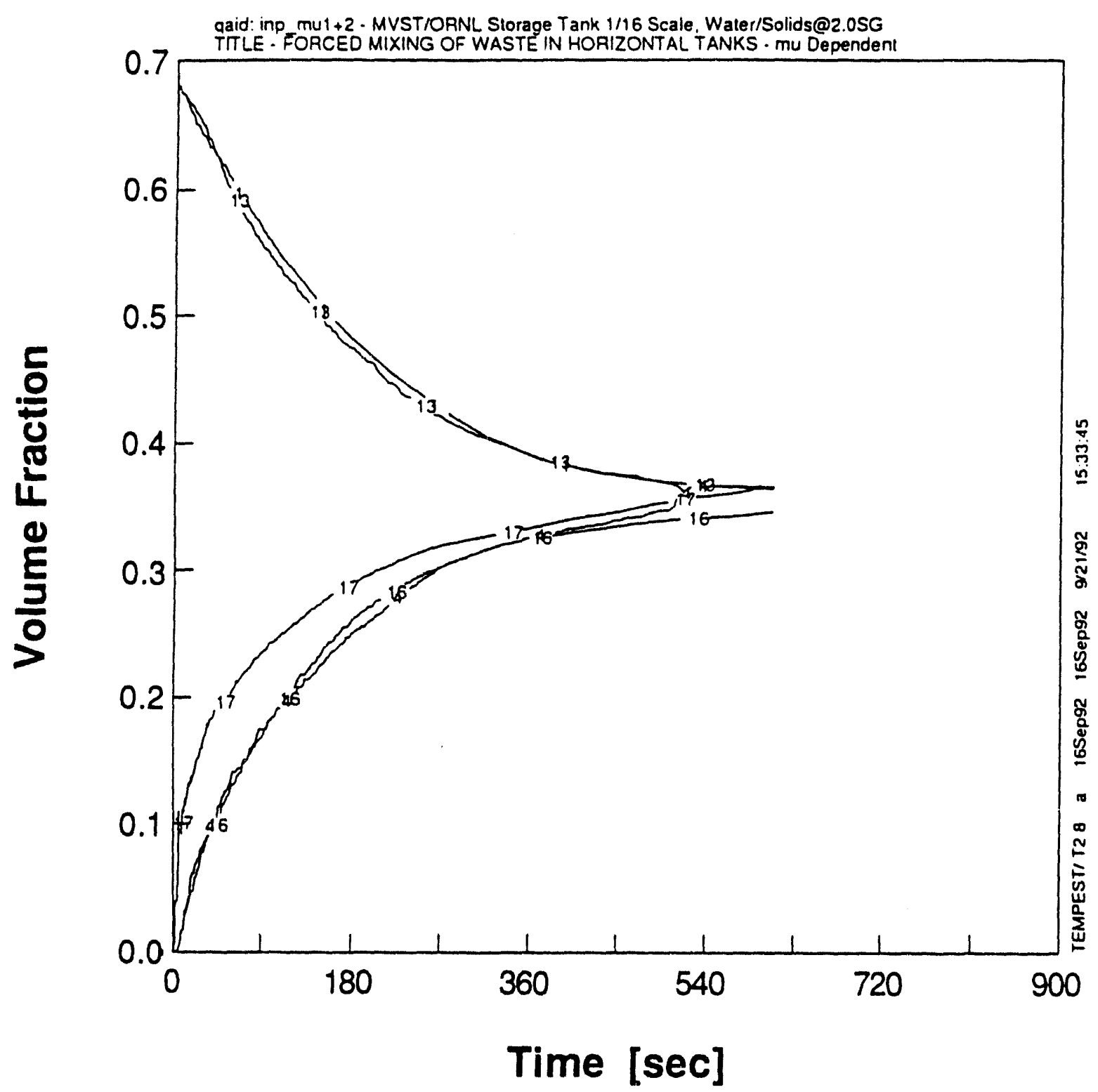

Figure 5.8. Time Dependence of Solids Concentration for Solid Particles in Water with an Exponential Viscosity Shape Function. Maximum Viscosity Ratio of $10^{2}$ and Effectively no Molecular and No Turbulent Diffusivity (Case 3b) 


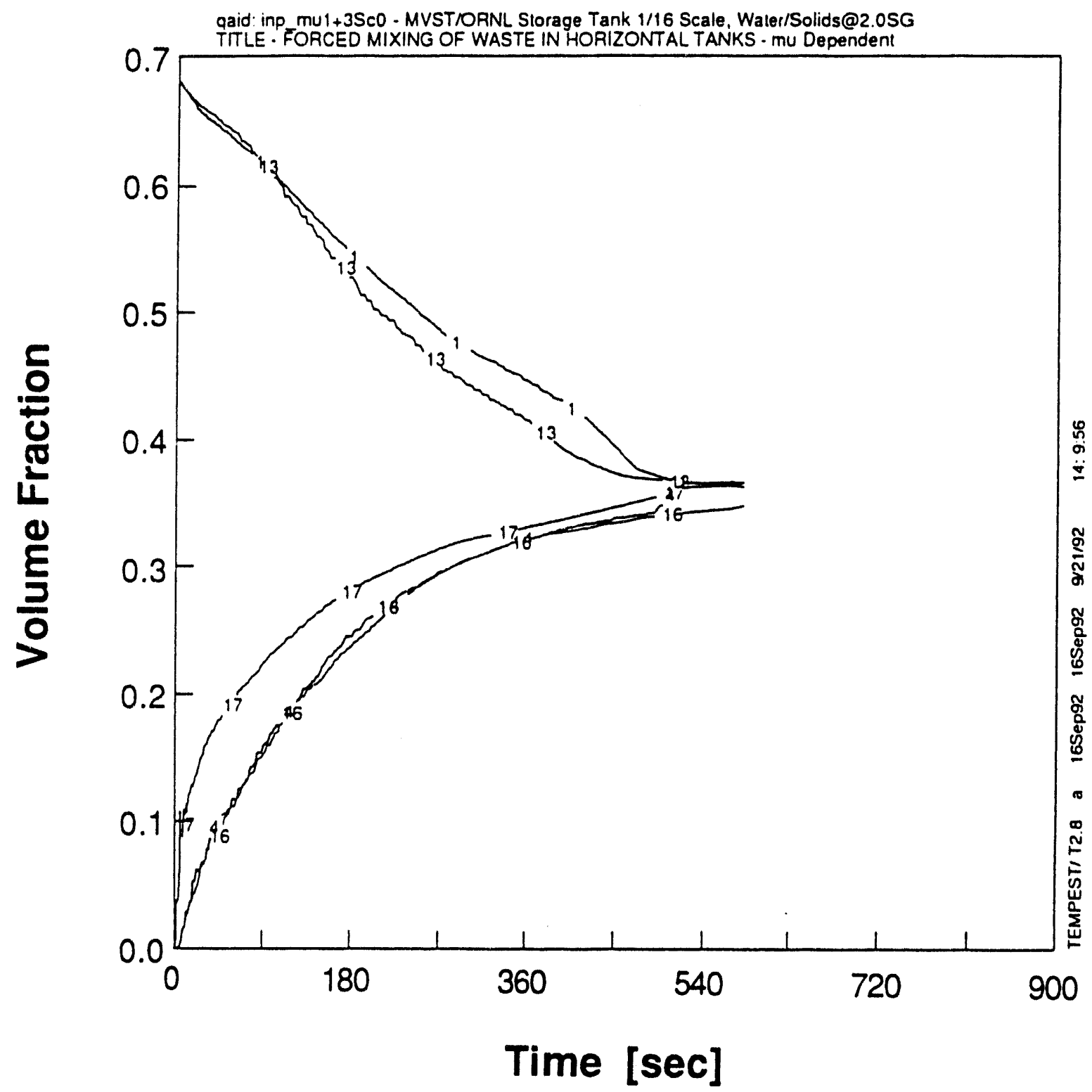

Figure 5.9. Time Dependence of Solids Concentration for Solid Particles in Water with an Exponential Viscosity Shape Function. Maximum Viscosity Ratio of $10^{3}$ and Effectively no Molecular and No Turbulent Diffusivity (Case 3c) 


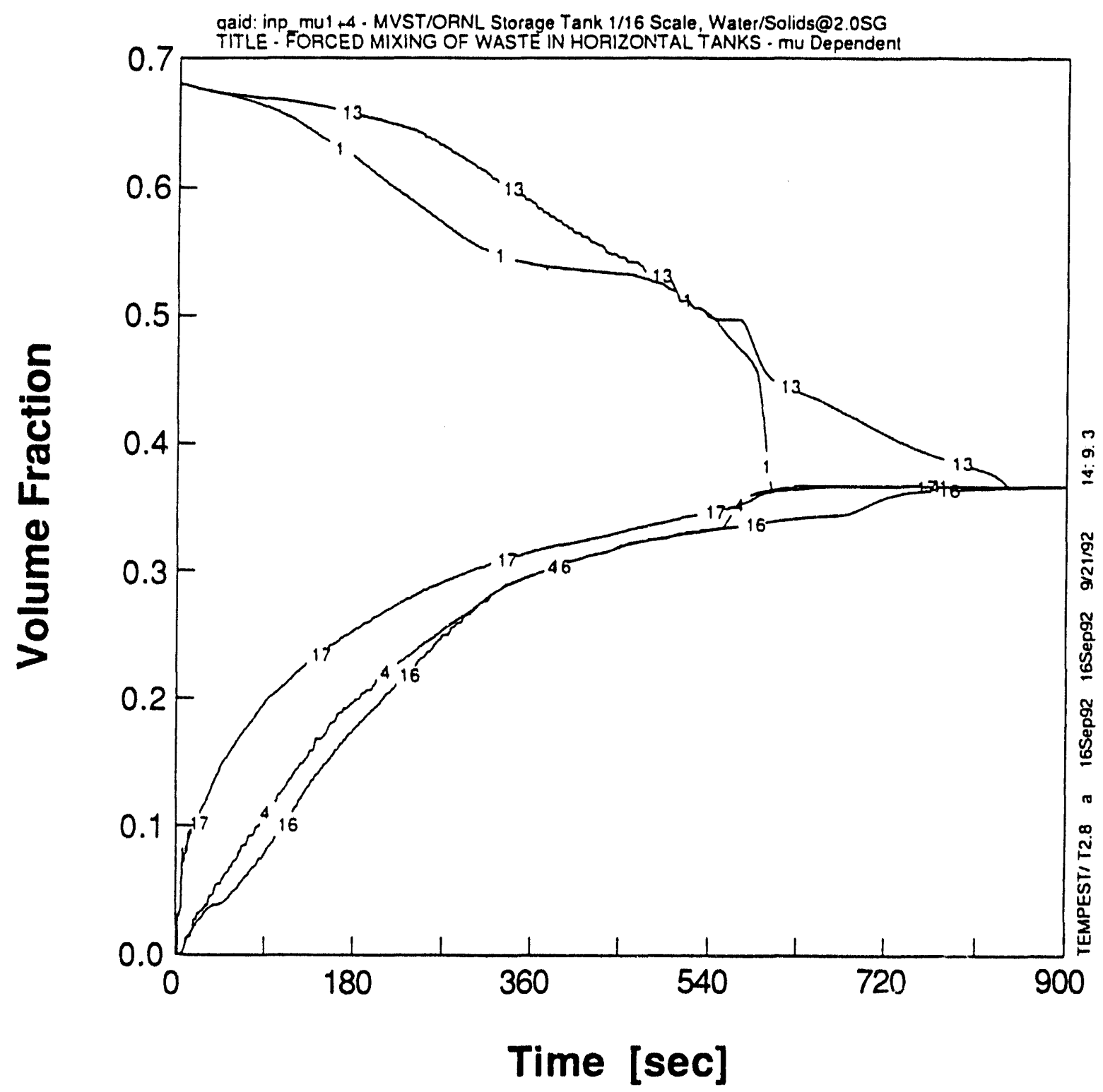

Figure 5.10. Time Dependence of Solids Concentration for Solid Particles in Water with an Exponential Viscosity Shape Function. Maximum Viscosity Ratio of $10^{4}$ and Effectively no Molecular and No Turbulent Diffusivity (Case 3d) 


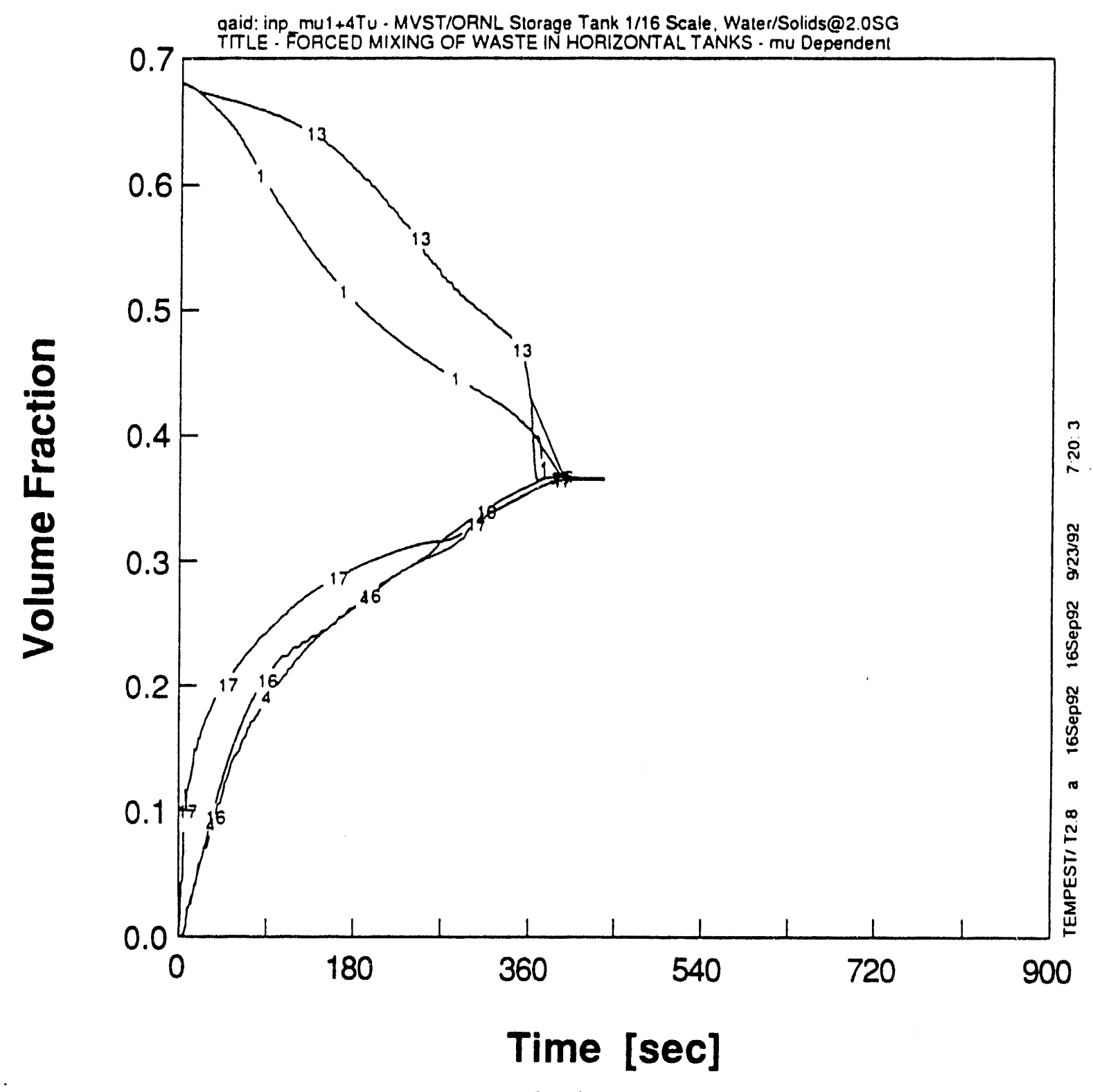

Figure 5.11. Time Dependence of Solids Concentration for Solid Particles in Water with an Exponential Viscosity Shape Function. Maximum Viscosity Ratio of $10^{4}$ and Effectively no Molecular and No Turbulent Diffusivity (Case 4d) 


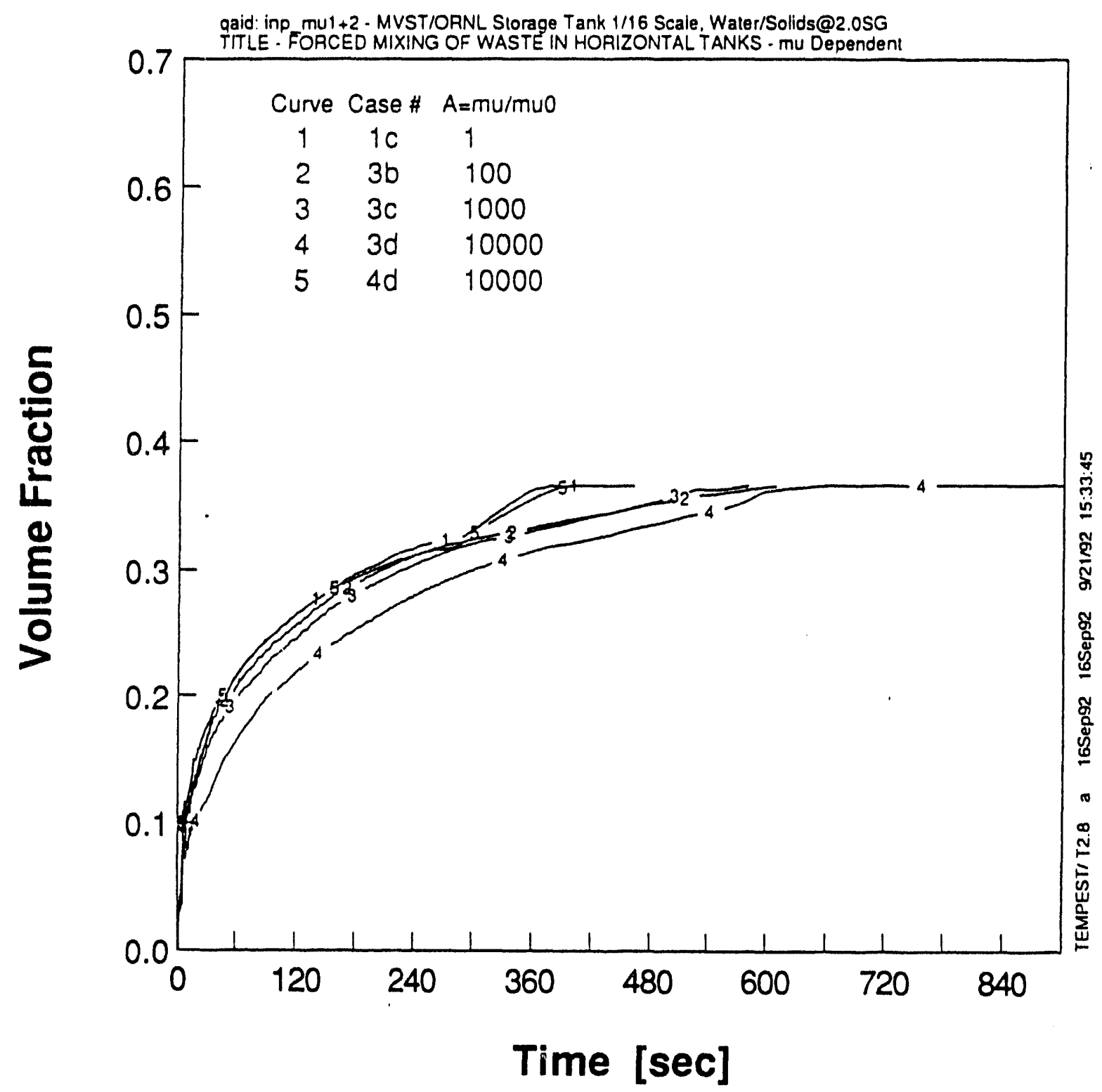

Figure 5.12. Composite of Time Dependence of Solids Concentration at Modeling Recirculating Pump Intake (Cases 1c, 3b-3d, and 4d) 
viscosity (slope of stress-strain curve) is large. Furthermore, the magnitude of the strain rate along a free jet axis is

$$
\dot{\gamma}=\sqrt{2}\left|\frac{\partial u}{\partial x}\right|
$$

Using the experimental correlations for the velocity along the jet axis (Rajaratnam 1976)

$$
u=5.75 \frac{U_{j} d}{x}
$$

the magnitude of the strain rate can be rewritten as

$$
\dot{\gamma}=8.1372 \frac{\mathrm{U}_{\mathrm{j}} \mathrm{d}}{\mathrm{x}^{2}}
$$

where $U_{j}$ is the jet velocity and $d$ is the jet diameter. In the case of the $1 / 6$-scale tank (length $=10 \mathrm{ft}$ ), when the jet velocities and diameters are $9 \mathrm{ft} / \mathrm{s}$ and $1 \mathrm{in}$., respectively, half of the tank's length along the jet axis will have strain rates less than $1 \mathrm{sec}^{-1}$, while the other half will have strain rates greater than $1 \mathrm{sec}^{-1}$. Thus, the choice of this strain rate is consistent with expected strain rates in the tank. For a relatively high effective viscosity, the computations result in conservative mixing time estimates.

To construct the concentration-dependent power-shape-viscosity model, the following procedure is used:

1. Obtain the instantaneous viscosity by taking the derivative of the shear stress with respect to the strain rate from the concentration-dependent power-law model for sludge W-28. This can be written as:

$$
\frac{\partial \tau}{\partial \dot{\gamma}}=\mathrm{m}\left(\mathrm{C}_{\mathrm{r}}\right) \mathrm{n}\left(\mathrm{C}_{\mathrm{r}}\right) \dot{\gamma}^{\mathrm{n}\left(\mathrm{C}_{\mathrm{r}}\right)-1}
$$

2. From the above expression, calculate the viscosity at six different values of $C_{r}$, with the strain rate fixed at $1 \mathrm{sec}^{-1}$. 
3. Use the viscosity values calculated in (2) to perform a least squares fit of a concentrationdependent power-shape-viscosity model expressed as:

$$
\mu=\mu_{\mathrm{o}}\left(1+\mathrm{a} \mathrm{C}_{\mathrm{r}}^{\mathrm{b}}\right)
$$

The least squares fit yiclds the following constants:

$$
\begin{aligned}
\mu_{\mathrm{o}} & =2.2 \times 10^{-3} \mathrm{~Pa} \mathrm{sec} \\
\mathrm{a} & =1647.1 \\
\mathrm{~b} & =1.8632
\end{aligned}
$$

Figure 5.13 presents the results of this mixing calculation. Included in the figure are the time-dependent concentrations for the monitor locations shown in Figure 5.2. Note that the concentrations at the far end of the tank (from the jet) differ by about $5 \%$ (curves 13 and 16) from those at the near end (curves 1 and 4; curve 17 is the recirculation line). This is because of the jet's inability to produce the same level of agitation at the far end as at the near end. It is expected, however, that eventually the tank will completely mix under this scenario, because the simulated solid particulate is not settling and because no apparent yield stress is contained in the model.

Several simulations were performed using the above model for several jet velocities, ranging from 1 to $10 \mathrm{ft} / \mathrm{sec}$. The results are plotted as mixing times in Figure 5.14. Two curves have been plotted to distinguish the time it takes to mix the near end and the far end of the tank. The concentration at each end of the tank is based on a pair of monitor cells (each pair located at opposite ends of the tank). The mixing time at the near end $T_{\text {near ond }}$ is obtained when the two concentrations at the monitor cells 1 and 4 satisfy the following equality

$$
2\left|\frac{C_{1}-C_{4}}{C_{1}+C_{4}}\right|=0.01
$$

Similarly the mixing time at the far end $T_{\text {far end }}$ is obtained when

$$
2\left|\frac{C_{13}-C_{16}}{C_{13}+C_{16}}\right|=0.01
$$




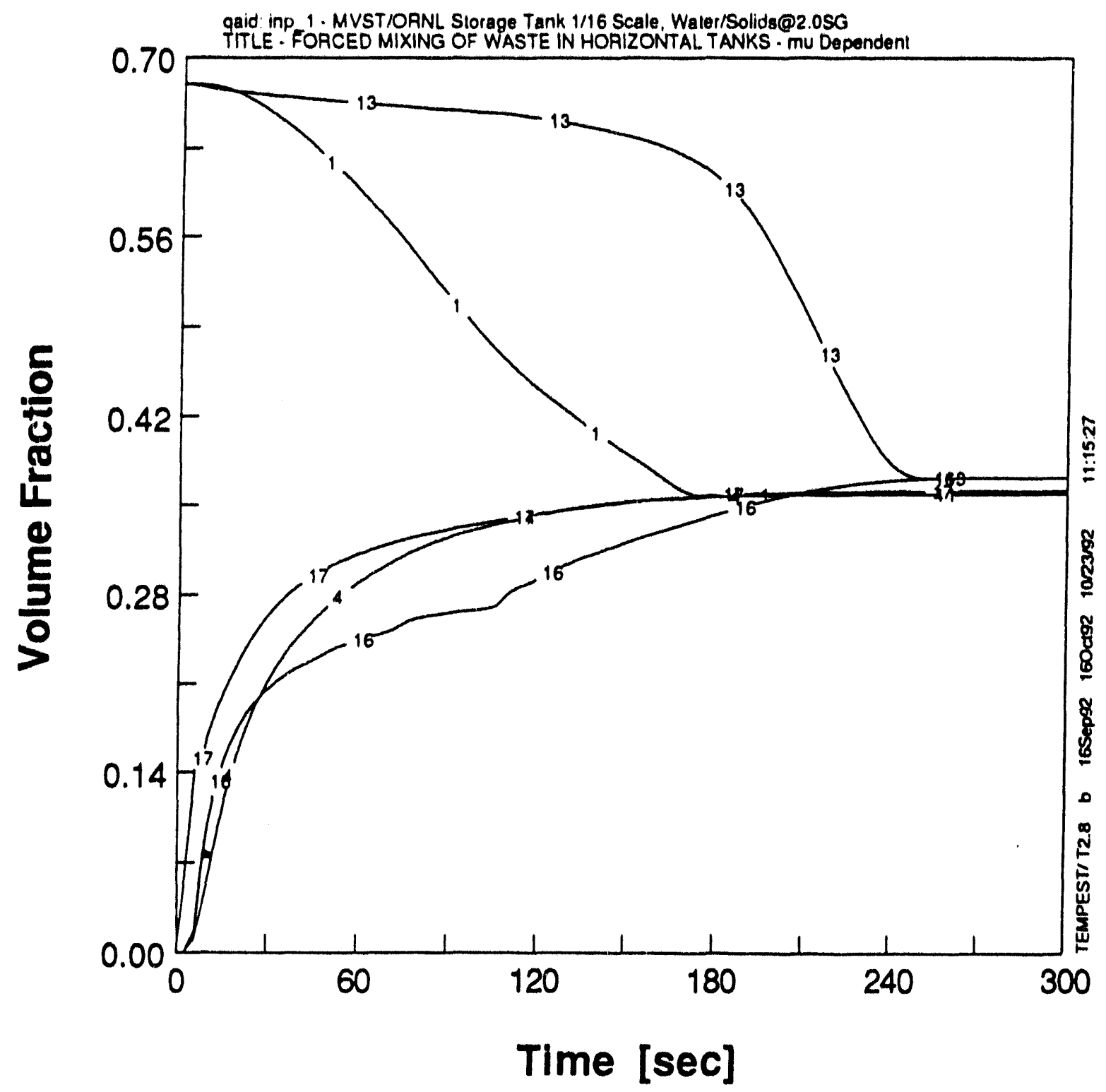

Figure 5.13. Volume Fraction as a Function of Time for a Concentration Dependent Power Shape Newtonian Viscosity Model with Dual Jet Velocity of $1 \mathrm{ft} / \mathrm{sec}$ 


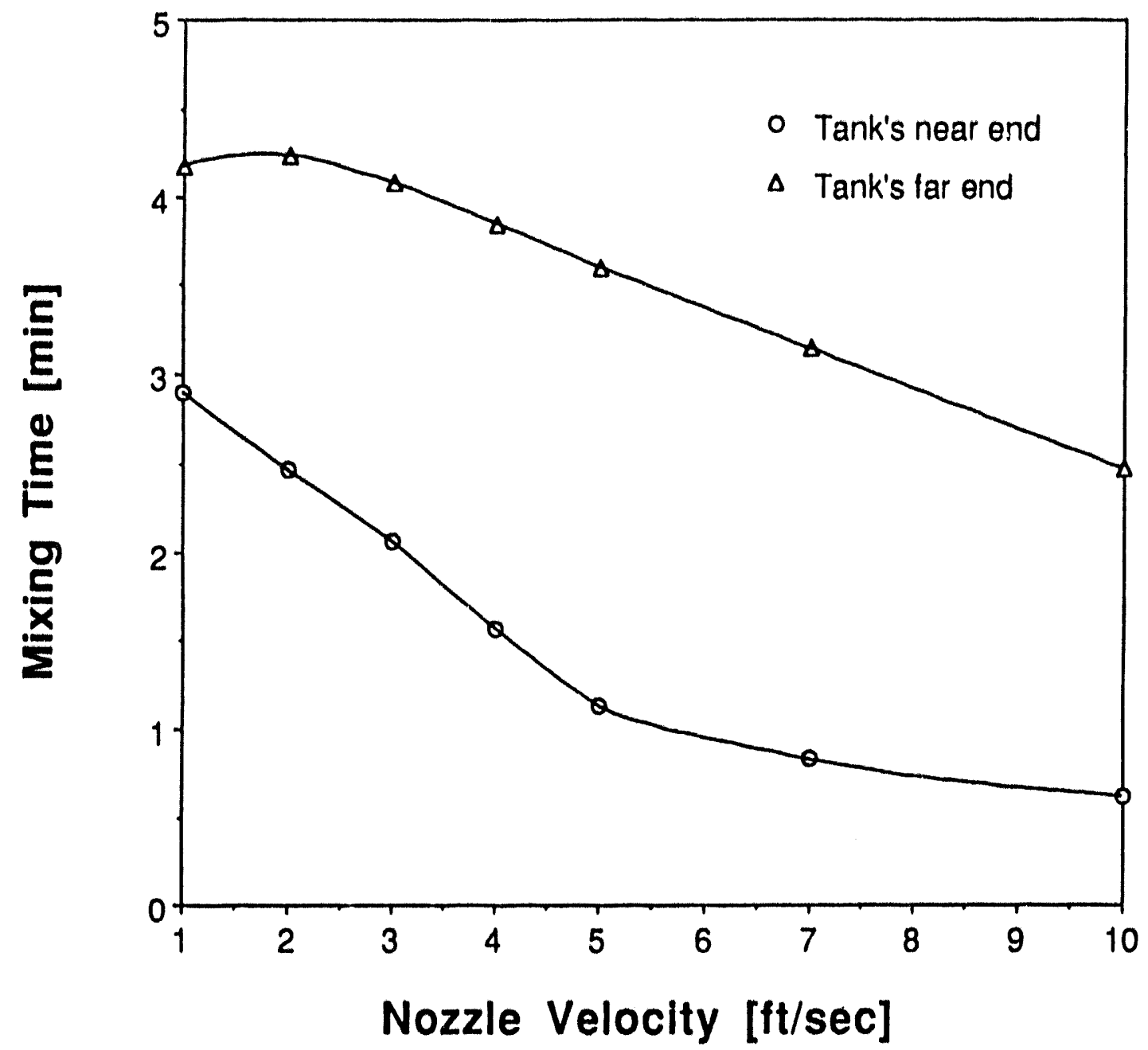

Figure 5.14. Mixing Times (at the near and far ends of the tank) as a Function of Jet Velocity for a Power-Shape Newtonian-Viscosity Model 
For this viscosity model and for the range of jet velocities analyzed, the mixing time at the far end was always greater than the mixing time at the near end. The mixing time at the far end is most representative of the time to mix the whole tank.

\subsection{Non-Newtonian Sludge Mixing Simulations}

The concentration-dependent power-law model developed in Section 4.1 is based on rheological data for the W-28 sludge waste and is used in simulations of mixing in a dual-jet 1/6-scale tank. The effective viscosity is given by

$$
\mu_{\text {eff }}=\left(A_{0}+A_{1} C_{r}+\Lambda_{2} C_{r}^{2}+A_{3} C_{r}^{3}\right) \dot{\gamma}^{\left[a^{-C_{r}}+\left(1-b^{-C_{r}}\right)\right]-1}
$$

where the model constants are presented in Section 4.1.2. In the simulations performed with this model, it was assumed that the specific gravity of solids is 1.56 and that of the supernatant is 1 .

The solid phase of the calculation was assumed to be a hindered settling solid. A Stoke's regime hindered-settling correlation (Perry and Chilton 1973) was applied:

$$
V_{s}=V_{\infty}\left(1-C_{r}\right)^{4.65}
$$

where $\mathrm{V}_{\mathrm{s}}$ is the hindered settling velocity, and $\mathrm{V}_{\infty}$ is the unhindered particle settling velocity. The value used was determined from the Stokes settling velocity expression:

$$
\mathrm{V}_{\infty}=\frac{2}{18} \mathrm{~d}_{\mathrm{p}}^{2} \mathrm{~g} \frac{\rho_{\mathrm{s}}-\rho_{\ell}}{\mu}
$$

$$
\text { where } \begin{aligned}
\rho_{s} & =\text { solid density } \\
\rho_{\ell} & =\text { liquid density } \\
\mu & =\text { liquid dynamic viscosity } \\
\mathrm{g} & =\text { gravitational acceleration } \\
\mathrm{d}_{\mathrm{p}} & =\text { particle diameter }
\end{aligned}
$$

A particle diameter of $10 \mu \mathrm{m}$ was used in these calculations. At this particle size, settling of solids particles is insignificant relative to computed mixing times. 
The numerical results obtained with this nonlinear effective viscosity model are fundamentally different from those for Newtonian fluids presented in Section 5.2. A remarkable feature is the formation of a sludge bank at the far end of the tank. For a dual jet configuration with velocity of $5 \mathrm{ft} / \mathrm{sec}$, the concentration time history curves (Figure 5.15) that correspond to monitor cells at the far end of the tank (13 and 16) show a wide departure from the average concentration indicated by the coalescence of curves 1,4 , and 17. In the same plot it can be seen that the near end of the tank becomes fully mixed in less than three minutes. The concentrations at the monitor locations 13 and 16 level off at a constant value which remains basically unchanged. The concentration at these far end monitor cell locations starts to slowly approach the average concentration as the jet velocity is increased, as shown in Figures 5.16 and 5.17. At a velocity of $20 \mathrm{ft} / \mathrm{sec}$, the transfer of momentum is high enough to achieve mixing throughout the tank. This can be seen in Figure 5.18, where all concentration curves come together after 3 minutes. These results are indicative of the existence of a critical jet velocity, below which a sludge bank can form.

The sludge bank formation process can be visualized from a time sequence of constant concentration contour plots. Figure 5.19 shows the velocity field as well as the concentration contours at one minute intervals. Variations in the contour plots are noticeable during the first three minutes, after which time the contours are unchanged. The contour line labeled 3 delineates the boundary of the region of sludge that cannot be mobilized. A reduction of the size of the sludge bank is accomplished by increasing the flow rate as seen in Figures 5.20 and 5.21 for jet velocities of 10 and $13 \mathrm{ft} / \mathrm{sec}$, respectively. However, at a velocity of $20 \mathrm{ft} / \mathrm{sec}$ no sludge bank is formed. In this case, after three minutes there is complete homogenization, as shown in Figure 5.22.

Figure 5.23 presents a composite perspective of these results for the jet velocities of 5,10 , 13 , and $20 \mathrm{ft} / \mathrm{s}$. In this figure, a contour surface of the average concentration is used as an indication of the jet's ability to fully penetrate the sludge layer. The dark color is the material which has not been mobilized. 


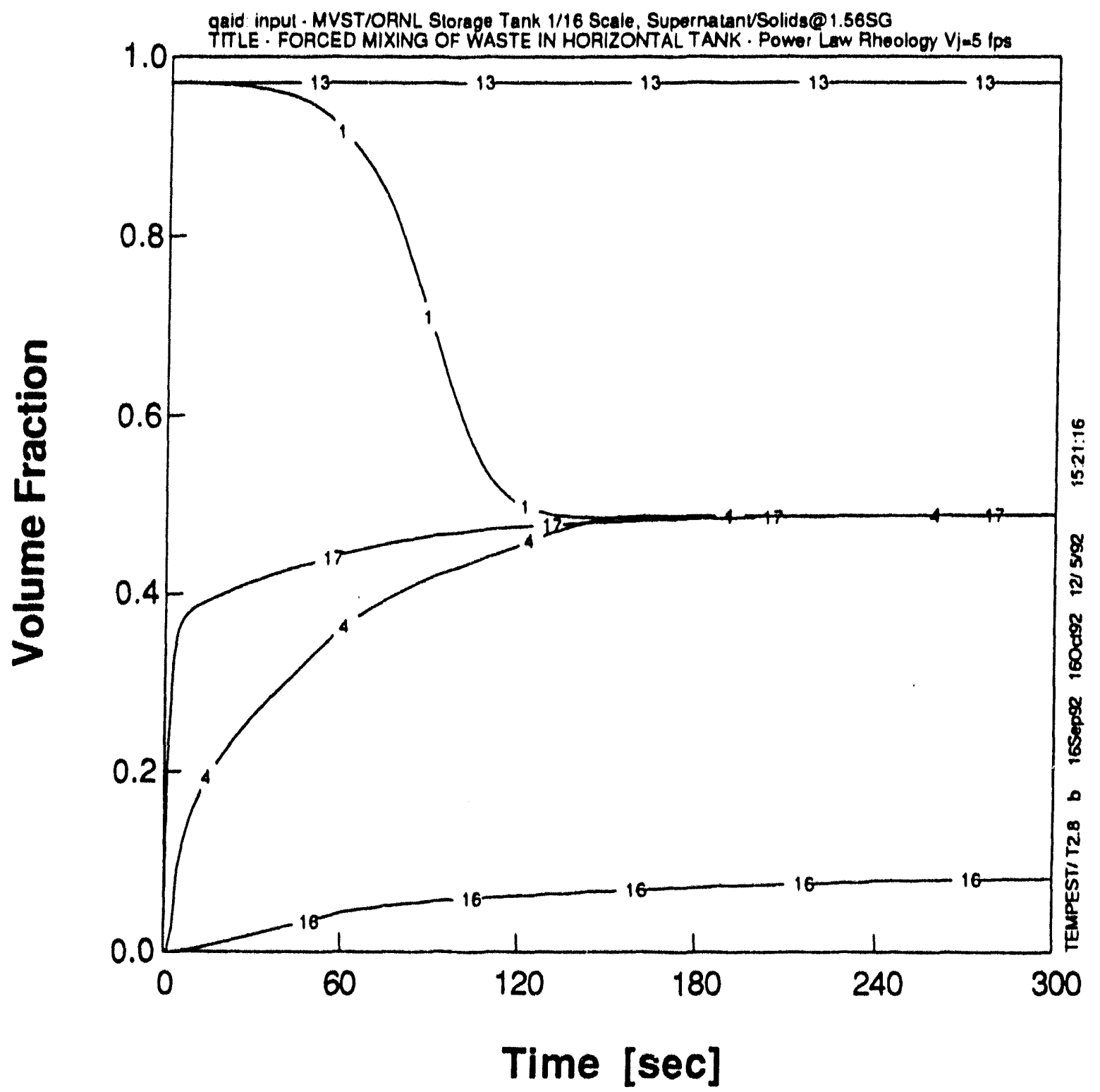

Figure 5.15. Volume Fraction as a Function of Time for a Concentration-Dependent Power-Law Model for the W-28 Sludge Waste with Dual Jet Velocity of $5 \mathrm{ft} / \mathrm{sec}$ 


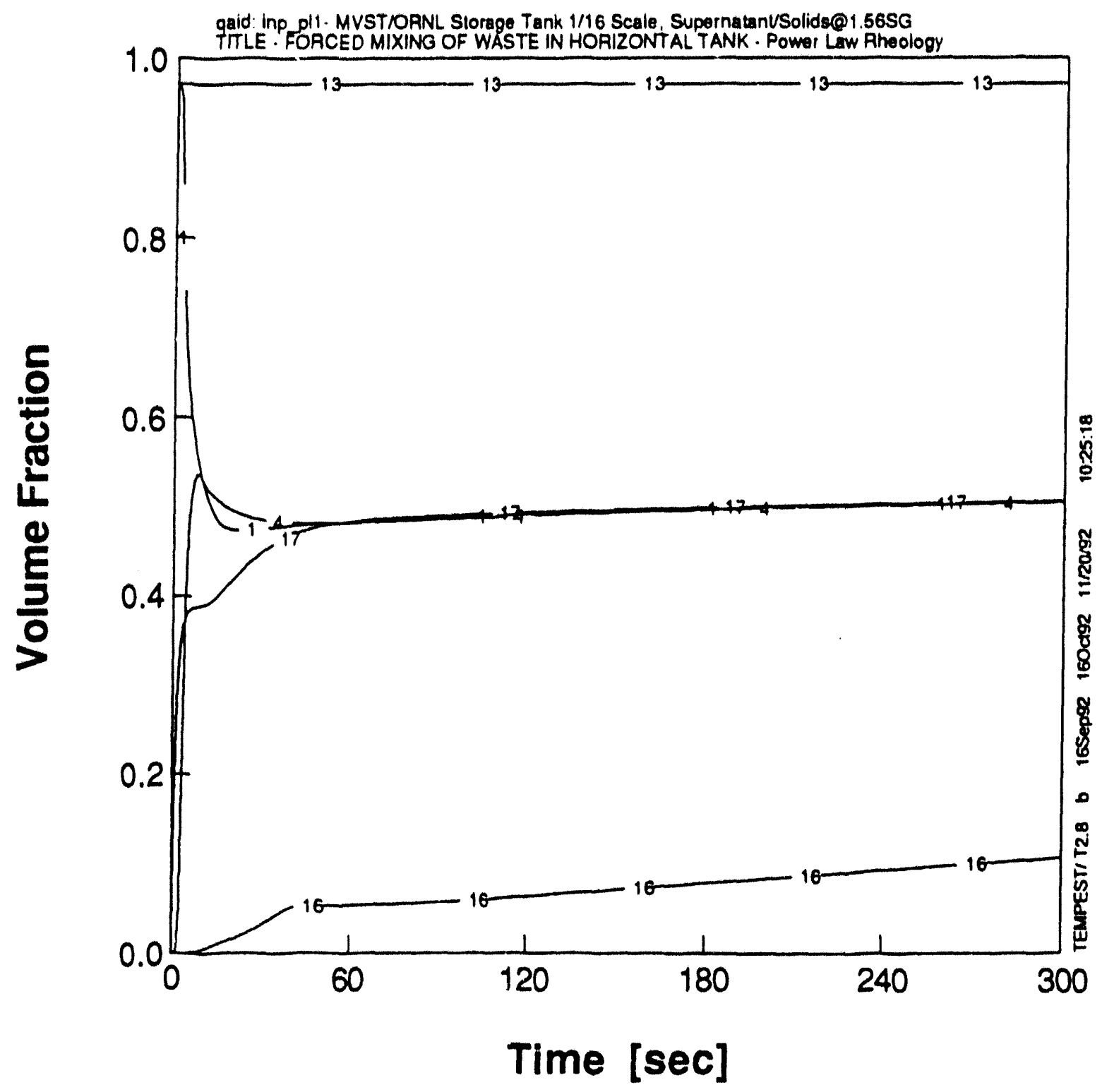

Figure 5.16. Volume Fraction as a Function of Time for a Concentration-Dependent Power-Law Model for the W-28 Sludge Waste with Dual Jet Velocity of $10 \mathrm{ft} / \mathrm{sec}$ 


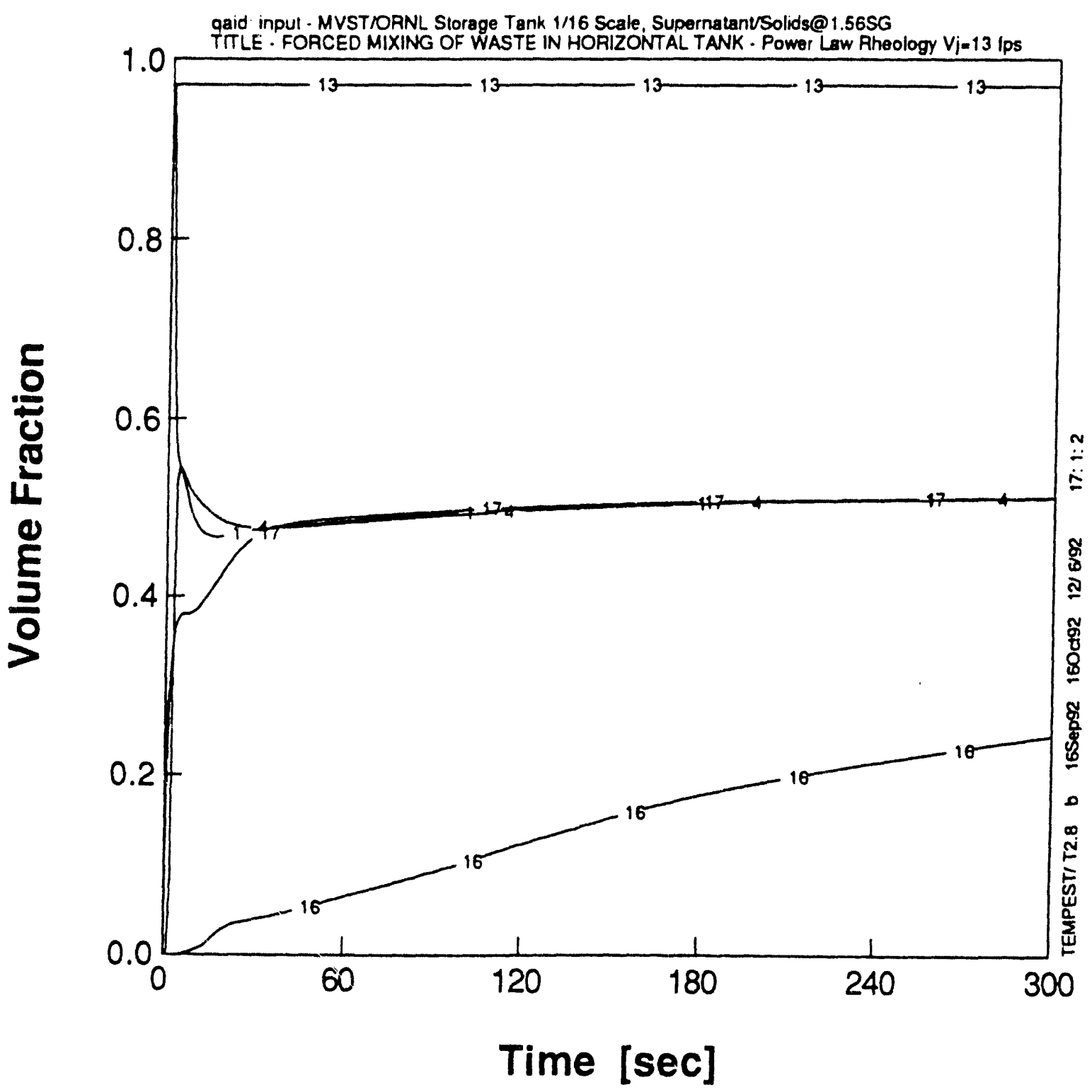

Figure 5.17. Volume Fraction as a Function of Time for a Concentration-Dependent Power-Law Model for the W-28 Sludge Waste with Dual Jet Velocity of $13 \mathrm{ft} / \mathrm{sec}$ 


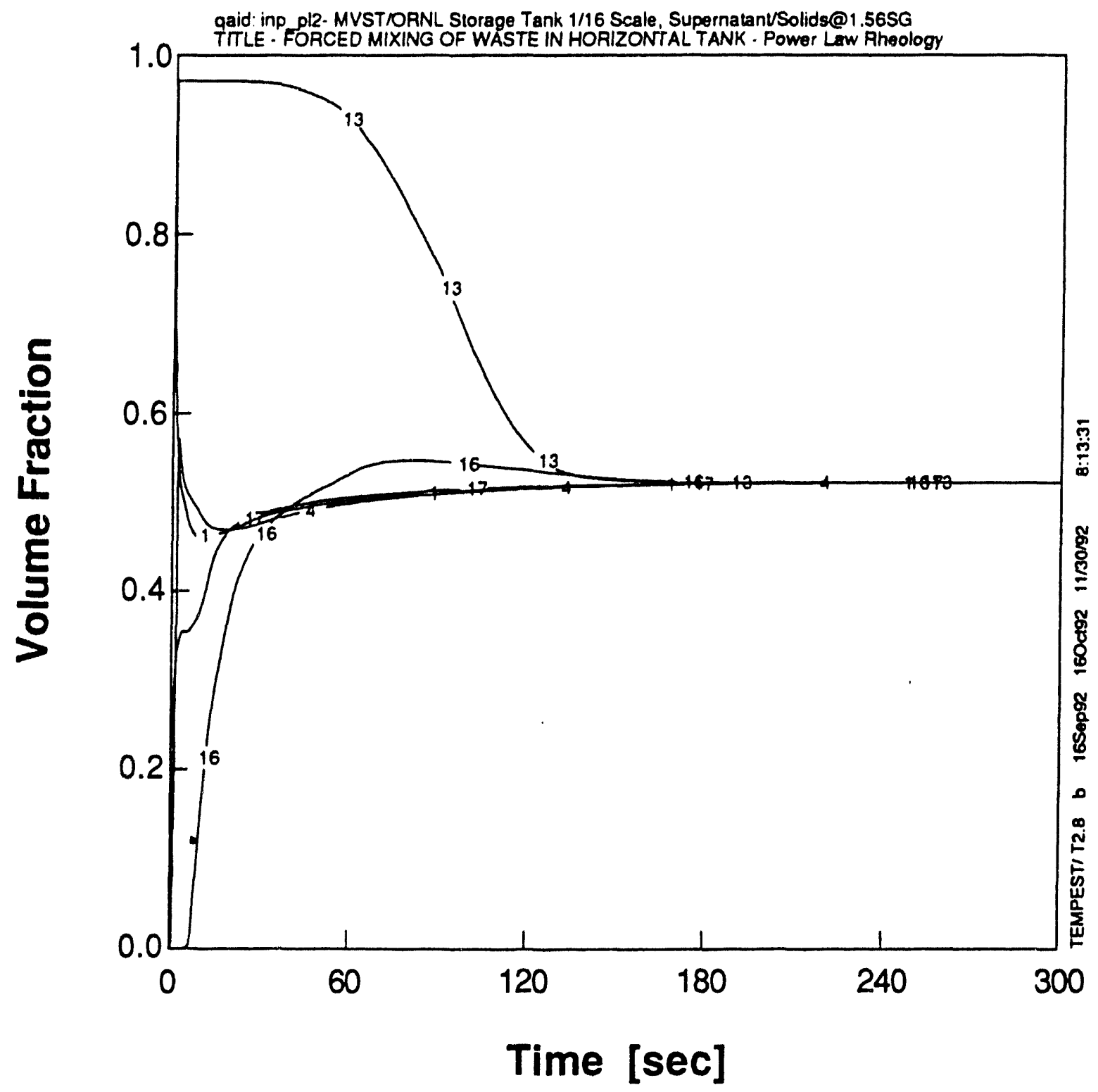

Figure 5.18. Volume Fraction as a Function of Time for a Concentration-Dependent Power-Law Model for the W-28 Sludge Waste with Dual Jet Velocity of $20 \mathrm{ft} / \mathrm{sec}$ Where Mixing Throughout the Tank has been Reached 
Time $=1 \mathrm{~min}$
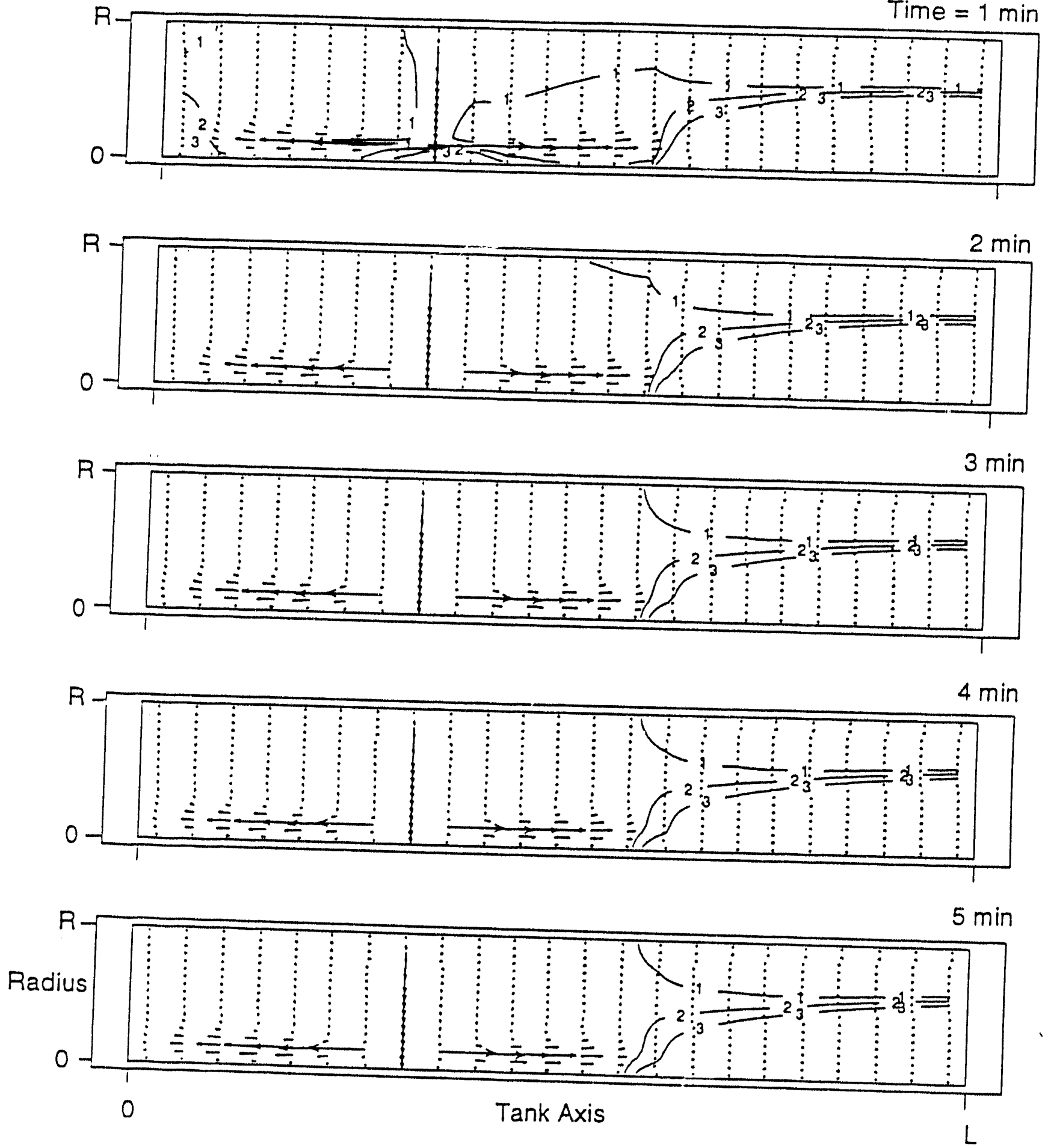

Figure 5.19a. Time Sequence of Velocity Fields and Mass Fraction Contours on the Plane of Symmetry of the 1/6-Scale Tank for a Concentration-Dependent Power-Law Model for the W-28 Sludge Waste with Dual Jet Velocity of $5 \mathrm{ft} / \mathrm{sec}$ 


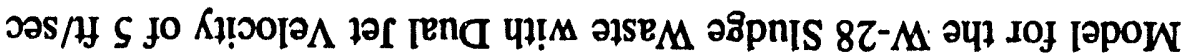

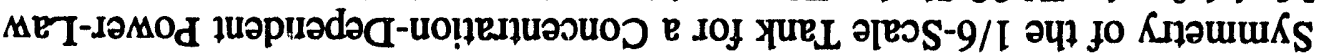

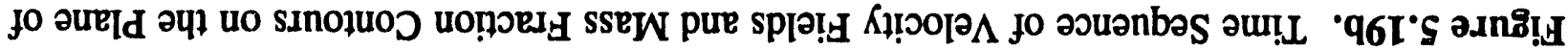

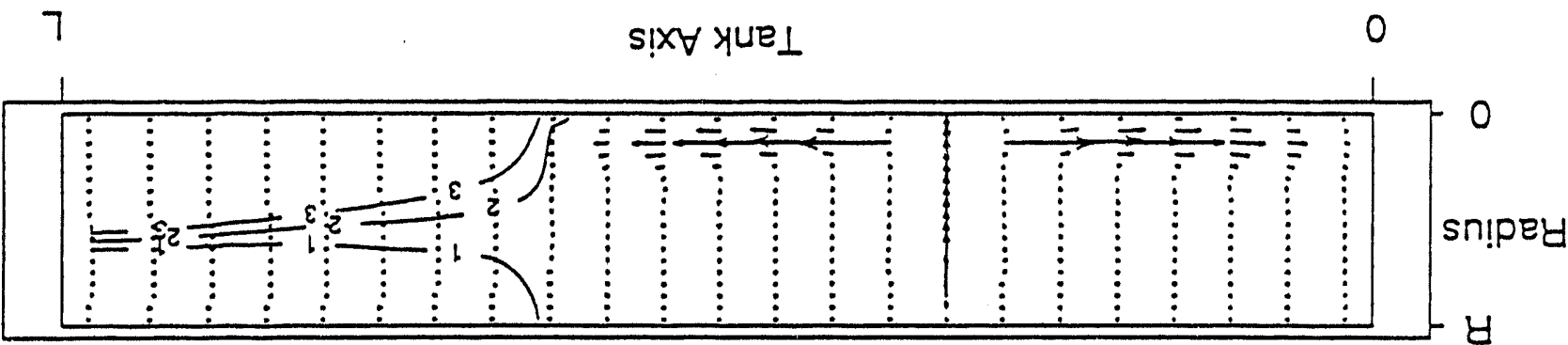

บ!̣ய Of

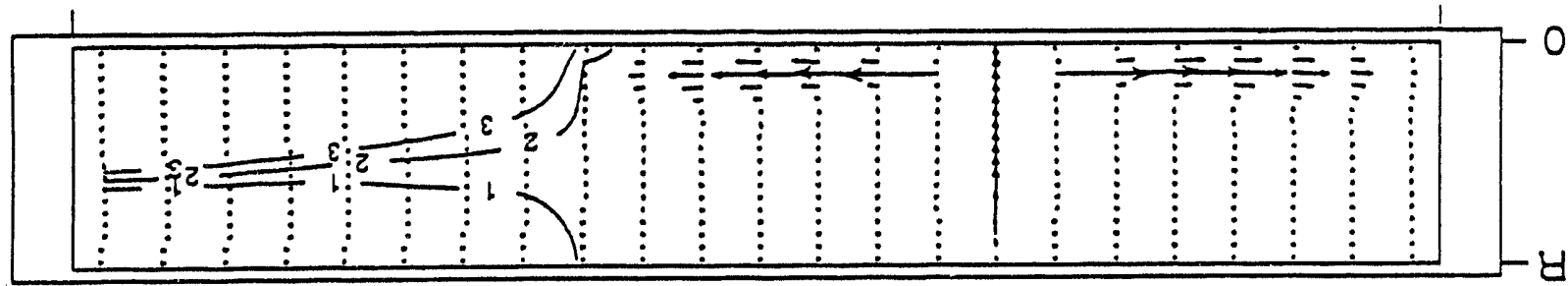

แ!ा 6

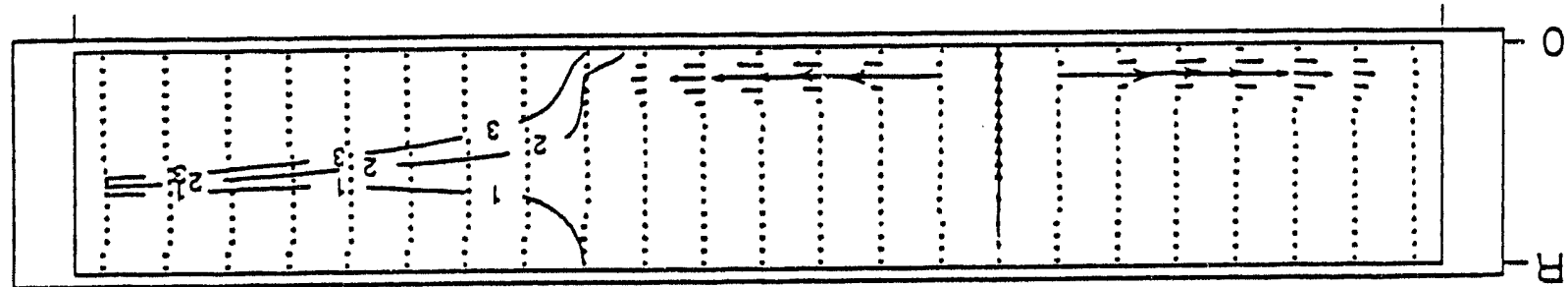

แ!แ 8

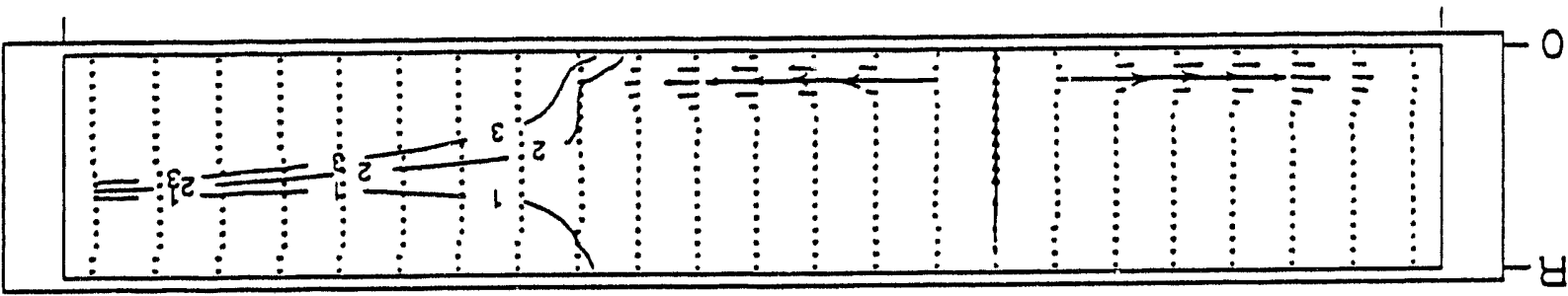

Uाए $L$

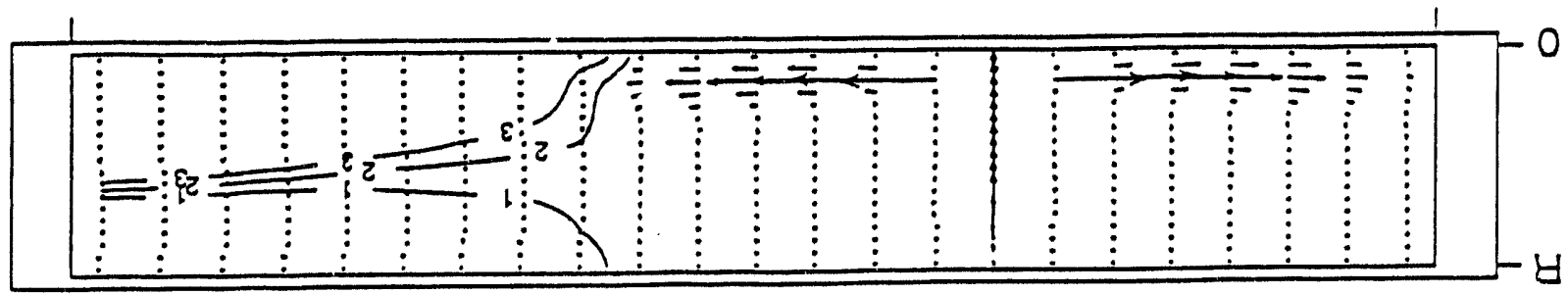

บ!พ 9 
Time $=1 \mathrm{~min}$
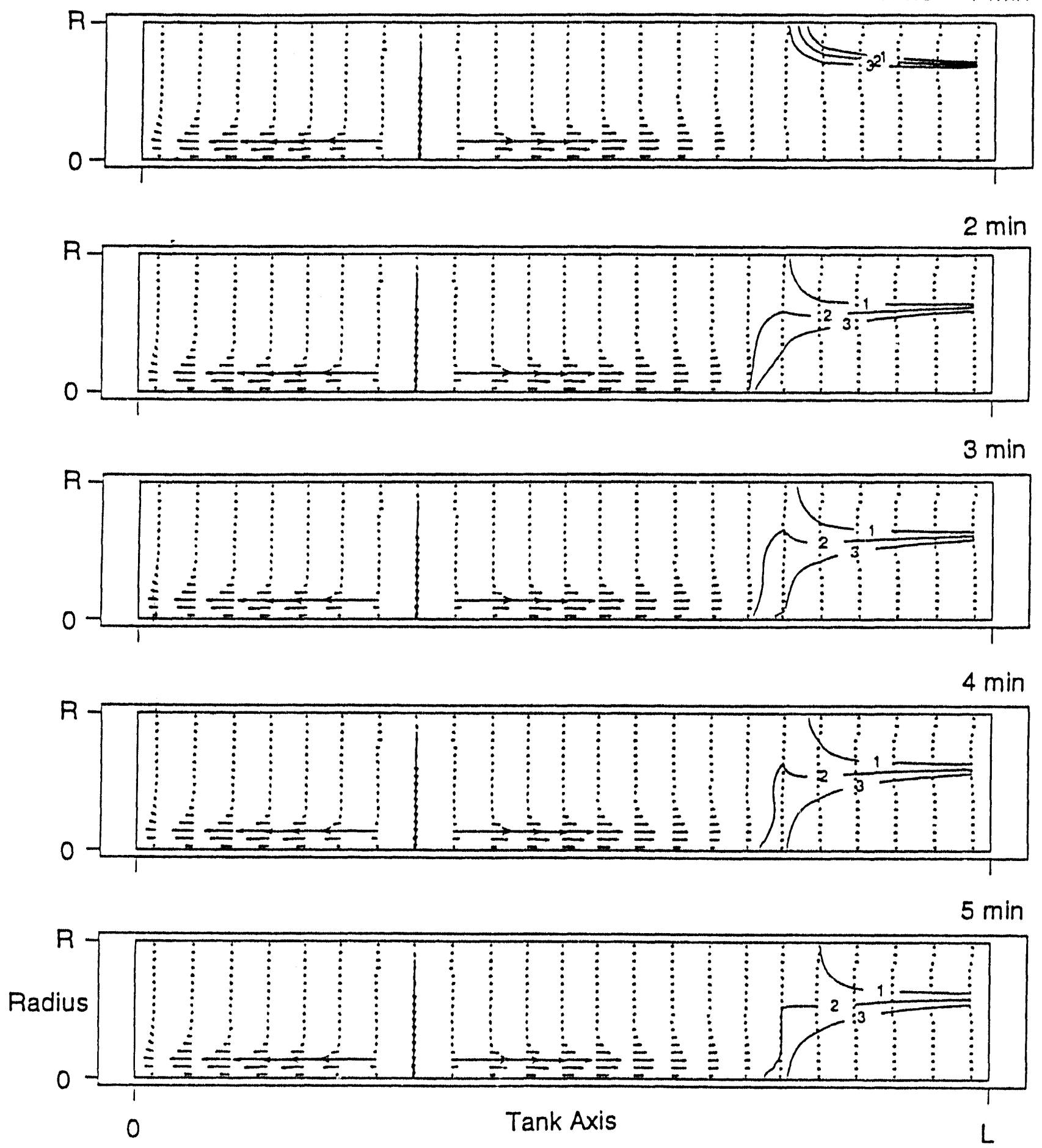

Figure 5.20a. Time Sequence of Velocity Fields and Mass Fraction Contours on the Plane of Symmetry of the 1/6-Scale Tank for a Concentration-Dependent Power-Law Model for the W-28 Sludge Waste with Dual Jet Velocity of $10 \mathrm{ft} / \mathrm{sec}$ 
$6 \mathrm{~min}$

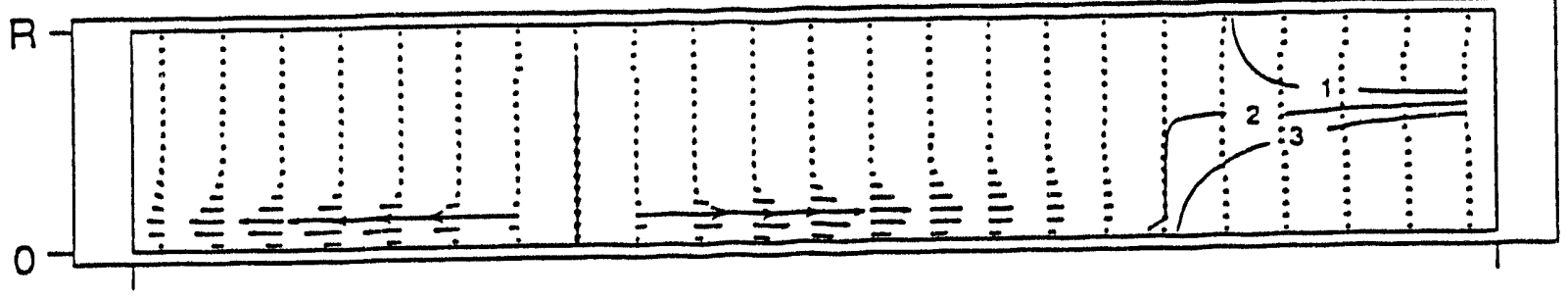

$7 \mathrm{~min}$

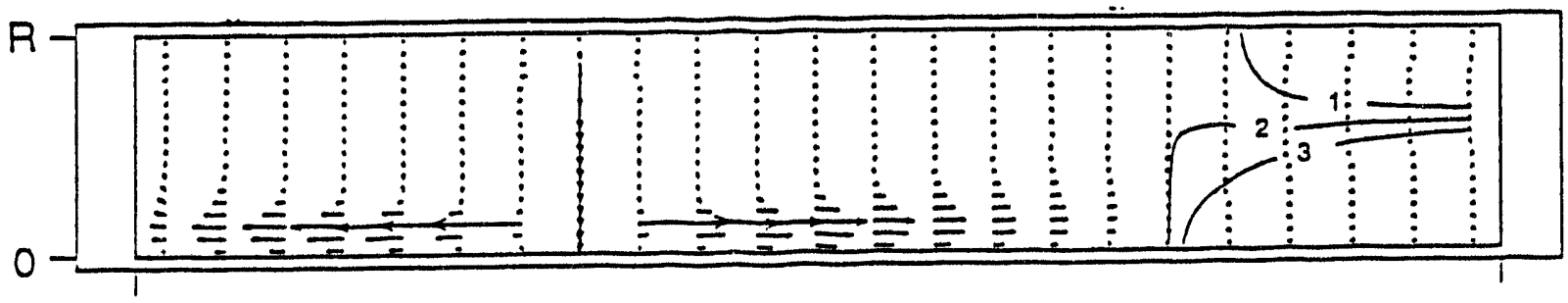

$8 \mathrm{~min}$
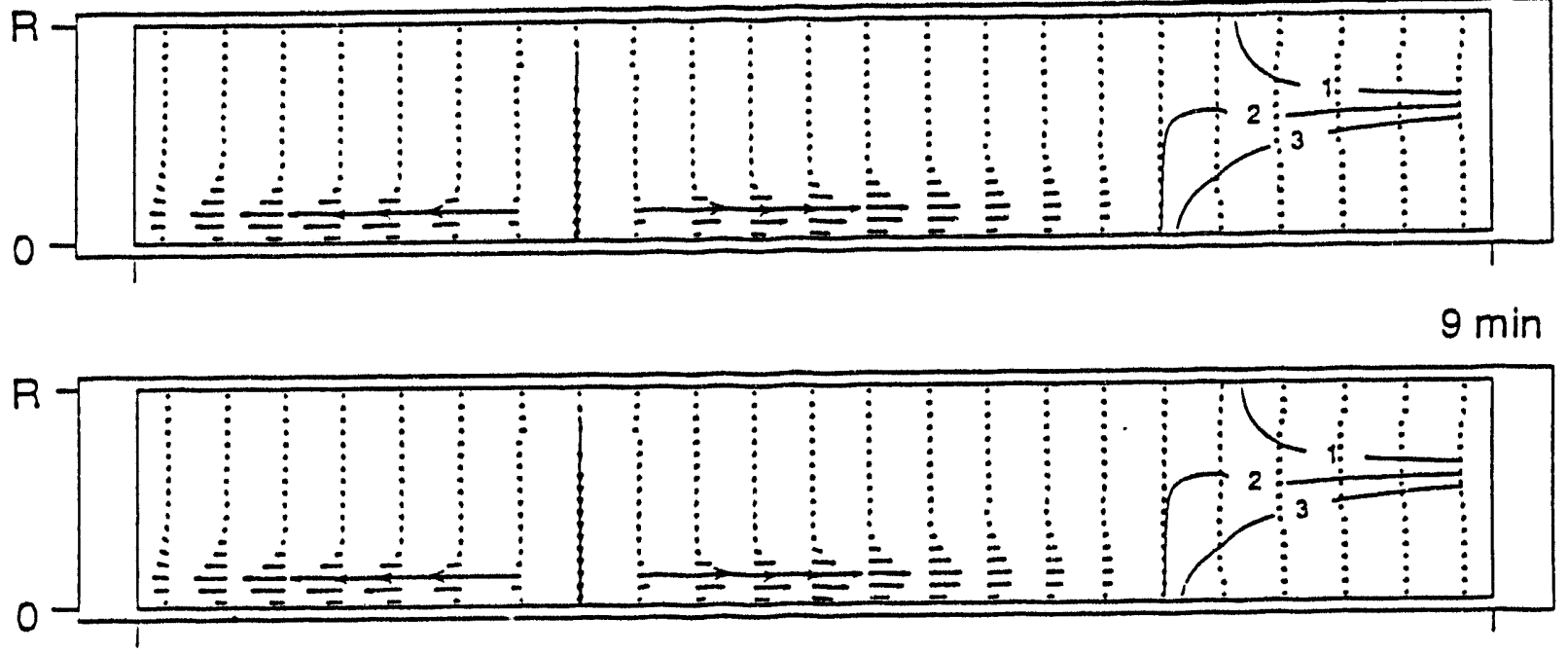

$10 \mathrm{~min}$

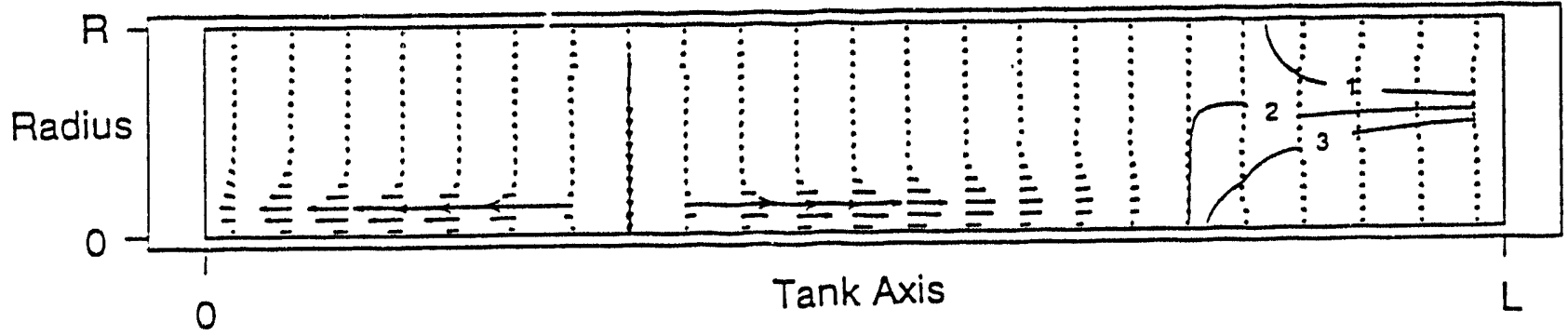

Figure 5.20b. Time Sequence of Velocity Fields and Mass Fraction Contours on the Plane of Symmetry of the 1/6-Scale Tank for a Concentration-Dependent Power-Law Model for the W-28 Sludge Waste with Dual Jet Velocity of $10 \mathrm{ft} / \mathrm{sec}$ 
Time $=1 \mathrm{~min}$
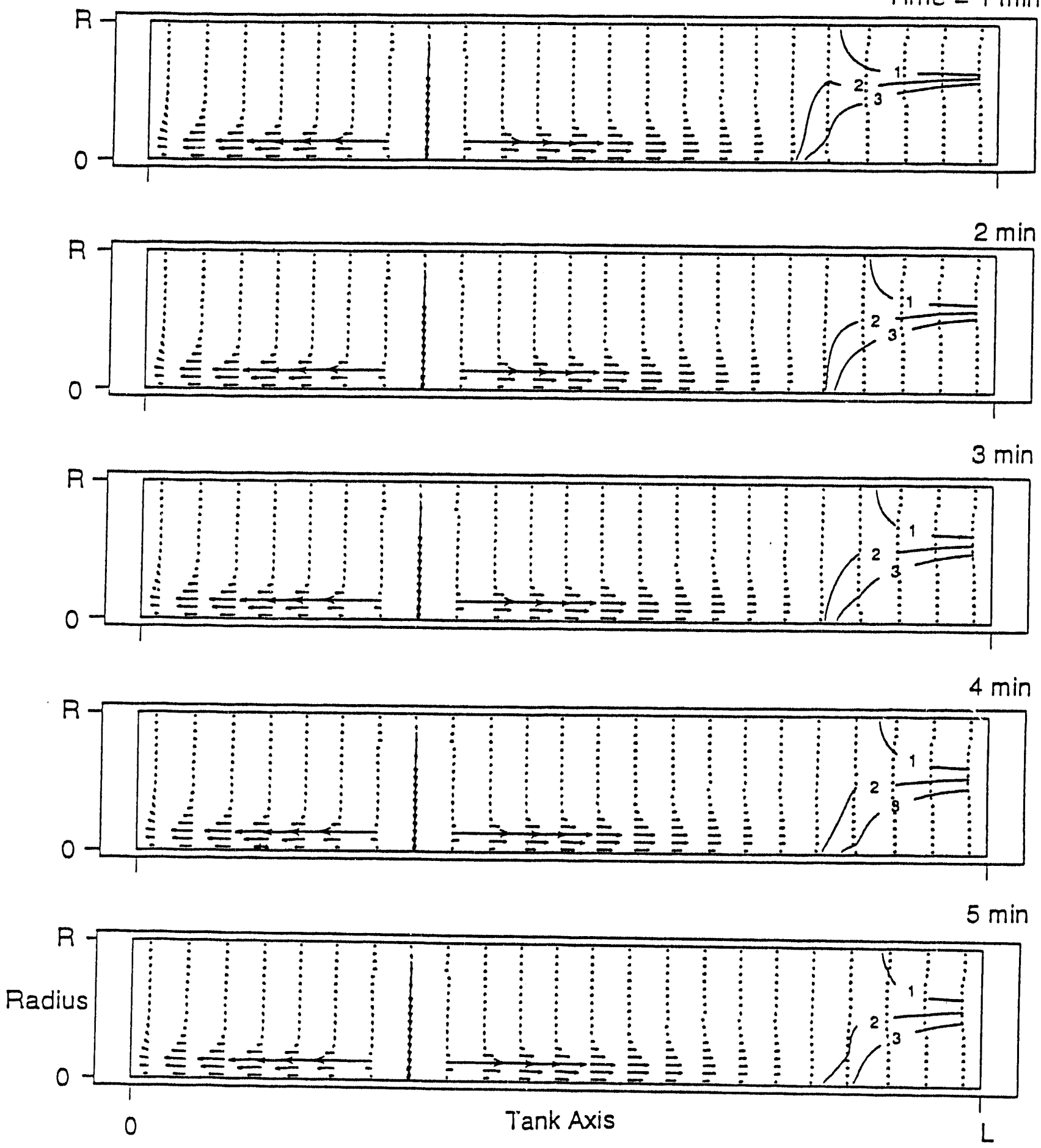

Figure 5.21a. Time Sequence of Velocity Fields and Mass Fraction Contours on the Plane of Symmetry of the $1 / 6$-Scale Tank for a Concentration-Dependent Power-Law Model for the W-28 Sludge Waste with Dual Jet Velocity of $13 \mathrm{ft} / \mathrm{sec}$ 

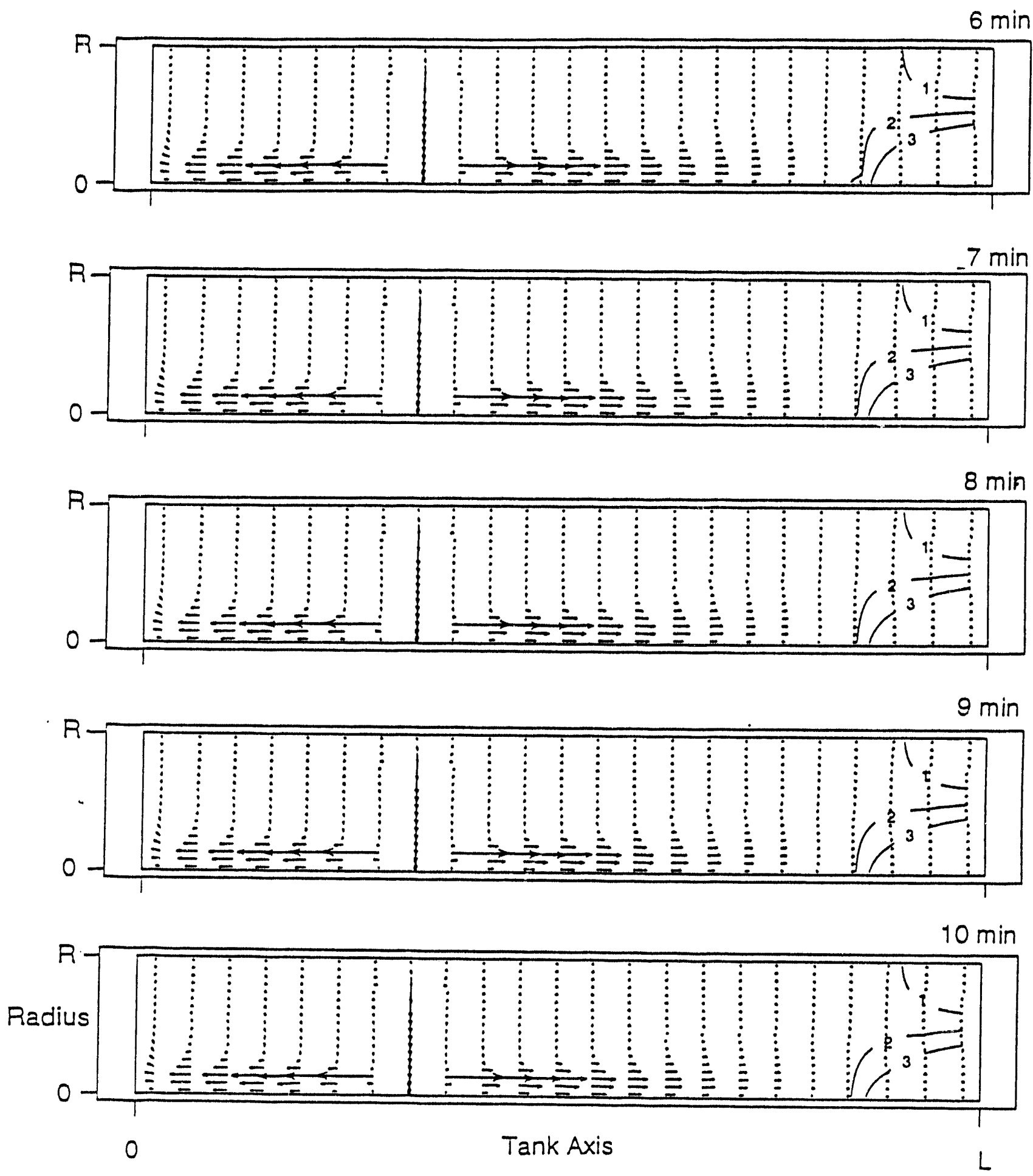

Figure 5.21b. Time Sequence of Velocity Fields and Mass Fraction Contours on the Plane of Symmetry of the 1/6-Scale Tank for a Concentration-Dependent Power-Law Model for the W-28 Sludge Waste with Dual Jet Velocity of $13 \mathrm{ft} / \mathrm{sec}$ 
Time $=1 \mathrm{~min}$
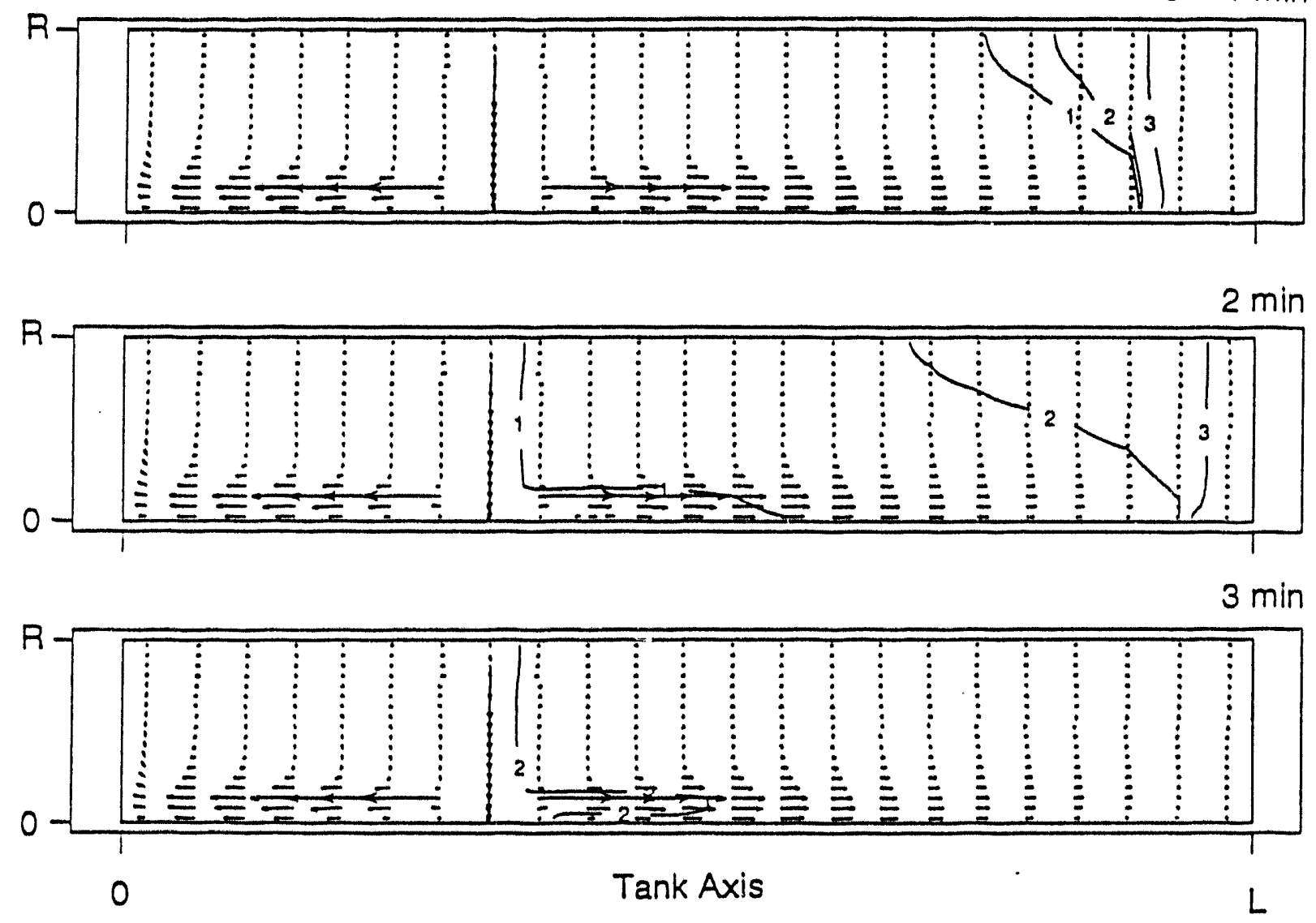

Figure 5.22. Time Sequence of Velocity Fields and Mass Fraction Contours on the Plane of Symmetry of the 1/6-Scale Tank for a Concentration-Dependent Power-Law Model for the W-28 Sludge Waste with Dual Jet Velocity of $20 \mathrm{ft} / \mathrm{sec}$ 


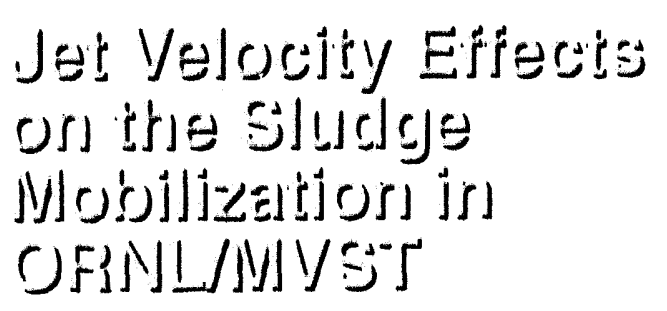

Jel Velocily eflects

ofs the slugese

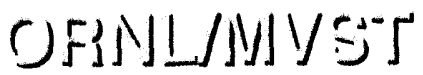

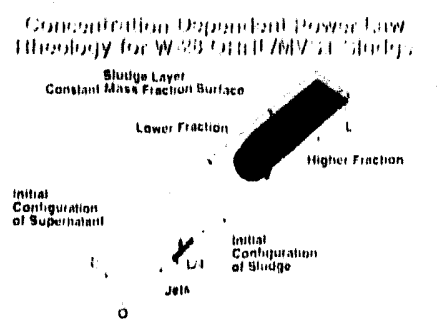

$V_{f, t}, 5 i t / 2, n$

$$
v_{j s t}=10 \mathrm{ft} /: \mathrm{ss}_{\mathrm{s}}
$$

$V_{1, t}: 1 ; 11 /, x_{i j}$

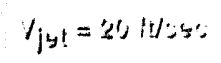

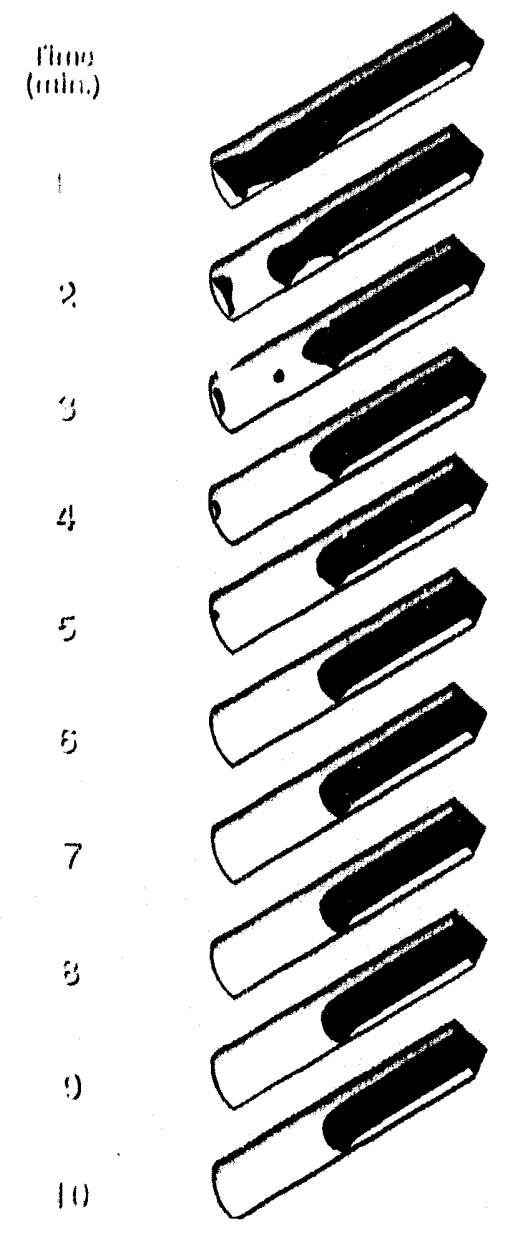

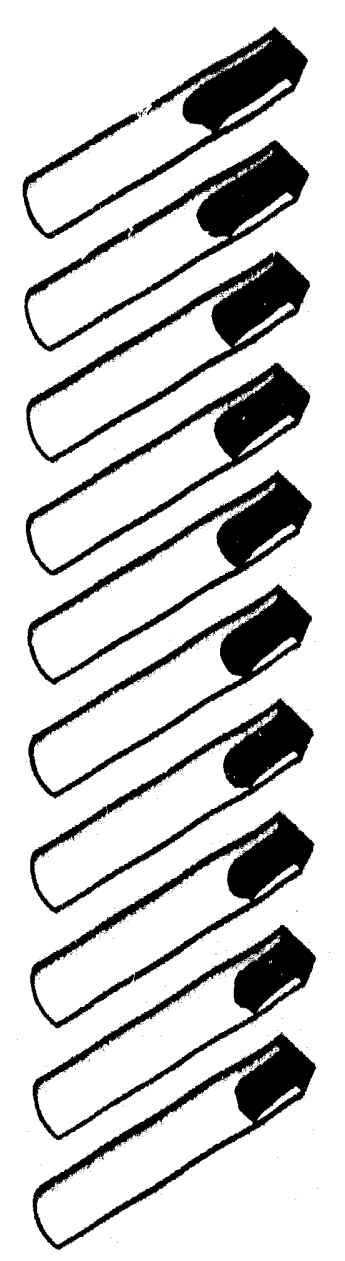

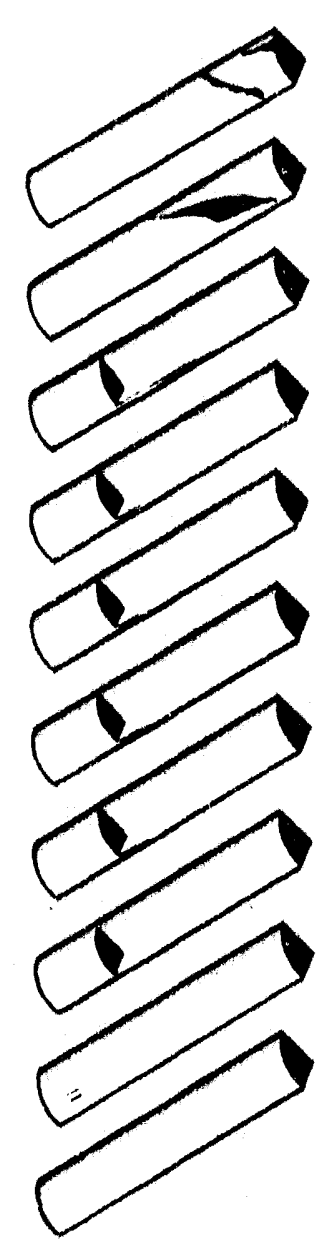

Figure 5.23. Jet Velocity Effects in MVST W-28 Sludge Mobilization-A Numerical Simulation at $1 / 6$ Scale 


\subsection{Saline Solution Mixing Simulations in a Filled Tank}

Numerical simulations of submerged jet mixing of a saline solution in scaled MVST tanks have been carried out. The objectives of these simulations are to test mixing criteria, to compute mixing times, and to compare computational results with preliminary experimental data. Water is the working fluid; dissolved salt is the tracer upon which mixing time determinations are based. Experiments were conducted at ORNL in a 1/6-scale mixing tank. The experience gained from these experimental and numerical studies will aid in understanding submerged jet mixing in the horizontal cylindrical tank configuration.

This section presents a discussion of mixing time criteria, computed mixing time results in 1/6- and 2/3-scale tanks, and comparison to preliminary experimental results.

\subsection{Mixing Time Criteria}

When a heterogeneous mixture contained in a vessel is being agitated by either moving impellers or by issuing jets, the concentration of the various components of the mixture is being varied at every point and at every instant within the vessel. For a given set of physical properties of the components comprising the mixture, relative amounts of solid and fluid components, jet velocities, and tank geometry, there will be an associated time after which the concentration at every point within the tank remains unchanged. When such a state of homogeneity has been reached, it is said that the mixture is fully mixed and the time it took to reach such a condition is the mixing time.

Ideally, a global criterion to determine the time at which full mixing is attained requires knowledge of the concentration time history over the entire fluid domain. For every point $\mathbf{r}$ within the fluid domain, a time $T_{r}$ can be assigned such that it satisfies the following conditions:

1. Concentration is everywhere equal to the average;

$$
C\left(r, T_{r}\right)=\bar{C}
$$


2. Time rate of change in concentration is everywhere zero;

$$
\left.\frac{\partial C(r, t)}{\partial t}\right|_{t=T_{r}=0}
$$

where $\mathrm{C}$ is the concentration function, and $\bar{C}$ is the average concentration. A third condition ensures that $T_{r}$ is the minimum time for which the first two conditions are satisfied.

3. A minimum mixing time exists;

$$
\lim _{t \rightarrow T_{1}^{+}} C(r, t-\varepsilon) \neq C
$$

In the third condition, using the limit of $\mathrm{C}$ as time approaches $\mathrm{T}_{\mathrm{r}}$ from the right will become clearer from the discussion of the numerically computed concentration time histories. When the above three conditions are applied to all spatial points in the fluid domain, a function $f$ can be formed

$$
T_{r}=f(r)
$$

The global nixing time $T$ is the maximum of $T_{r}$ over the spatial domain.

$$
T=\max _{\mathbf{r}}\left[\mathbf{T}_{\mathbf{r}}\right]
$$

In defining this criterion, it was implicitly assumed that the mixture is homogenizable, i.e., an average concentration is tenable throughout the fluid domain. This is true for soluble mixtures, which is the case being treated in this section. For more complicated mixtures with insoluble solids which tend to settle, a generalization of the above global criterion is needed.

Clearly, it is not possible to monitor the concentration at an infinite number of points in the flow field, either numerically or experimentally. Numerically, the maximum number of monitor locations is limited by the number of discrete computational cells. The global criterion defined above can be discretized to predict mixing times computationally.

Alternatively, the time evolution of a distribution function of concentration can also be used to computationally determine the mixing time. A concentration distribution can be constructed by subdividing the concentration range into discrete incremental intervals. At 
each interval a value is assigned, which is equal to the number of cells whose concentration falls within that interval. Initially, the concentration distribution function is localized in two extremes of the concentration range, namely, the interval of concentration near zero (pure water) and the interval of maximum concentration (salty water). Once mixing has commenced the distribution will evolve towards a normal distribution whose peak is centered at the average concentration $\overline{\mathrm{C}}$. Theoretically, all computational cells will reach $\overline{\mathrm{C}}$ and the distribution will become a single peak. In practice, it is more appropriate to base a mixing criterion on the majority of computational cells $(95 \%$, say) falling within the average concentration interval ( $\overline{\mathrm{C}} \pm 2.5 \%$, say). Even though the global mixing criterion based on a concentration distribution does not require a major modification of the existing code, it was not used because the primary interest was in comparing numerical and experimental results.

Experimentally, the number of points used to establish the mixing time is very limited because only a very small number of concentration probes can be introduced in the tank without appreciably affecting the flow field. Therefore, it is desirable to monitor the concentration at locations where it is believed that the mass transport will not be very efficient. Choice of such measurement locations requires some judgement, which can be aided by visualization of mixing experiments. These locations will usually be far from the source of mixing (jet nozzle) and close to the boundaries of the container. A mixing time based on the concentration time history of arbitrarily chosen locations may not provide a consistent indication of homogenization. This is another reason that experiments and computations complement each other in scaled tests prior to performing an analysis at full scale.

In the salt-water mixing experiments performed at $O R N L$, only four conductivity probes were used to establish the mixing time. The probes were located at a variety of positions, in an effort to gain the best reproductibility and best estimate of mixing times. Soms experiments were conducted with the probes located near the tank ends and some with probes located elsewhere, based on visualization experiments. In some tests, a probe was placed in the recirculation line. In the numerical simulations, the concentration was monitored at five cells: four near the end of the tank (see Figure 5.2) and one at the jet itself. With these five cells, three mixing time criteria were defined. Because a global criterion cannot be established with so few cells, it should not be surprising that the various criteria yield different mixing times.

The mixing criteria are as follows. Let the concentration time history at each one of the five monitor cells used $(i=1,2,3,4,5)$ be

$$
C_{i}(t)=C\left(r_{i}, t\right)
$$

and the time when the $i-$ th monitor cell reaches $\bar{C}$ be denoted by $T_{i}$ 


$$
\mathrm{C}_{\mathrm{i}}\left(\mathrm{T}_{\mathrm{i}}\right)=\mathrm{C}
$$

\subsubsection{Criterion 1}

A mixing time can be calculated by determining the time for which the maximum difference in concentration is a prescribed fraction of the average value, $\overline{\mathrm{C}}$. This value is found by searching for the maximum concentration difference backwards in time starting at a point when all monitor cells have reached $\bar{C}$. Mathematically this criterion is expressed as the time for which the following equality is satisfied

$$
\frac{\max _{i, j}\left[\left|C_{i}\left(T_{i}-\tau_{i}\right)-C_{j}\left(T_{j}-\tau_{j}\right)\right|\right]}{C}=\delta
$$

where $\delta$ is a prescribed percentage departure from $\bar{C}$, and $\tau_{i}$ is such that $T_{i}-\tau_{i}=T_{j}-\tau_{j}$ for $i \neq j$. Note that once $\delta$ has been chosen, $C_{i}$ is evaluated at a time for which the difference $T_{i}-\tau_{i}$ is a minimum.

\subsubsection{Criterion 2}

A second criterion can be based on a percent departure between the average value $\bar{C}$ and the concentration at the jet nozzle (monitor cell 5). The rationale behind this criterion is that the turbulent flow produced by the submerged jet will force all fluid elements within the tank to pass through the jet nozzle. Hence, the concentration at this monitor cell is indicative of the degree of mixing in the tank. Mathematically, this criterion is expressed as the time for which the following equality is satisfied

$$
\frac{\left|\mathrm{C}-\mathrm{C}_{5}\left(\mathrm{~T}_{5}-\tau_{5}\right)\right|}{\mathrm{C}}=\delta
$$

As before, to establish this mixing time, the concentration difference must be traced backwards in time to ensure that $T_{5}-\tau_{5}$ is the minimum time difference.

\subsubsection{Criterion 3}

A third criterion can be based on departures from $\bar{C}$ at the near (monitor cells 1 and 2 ) and far end (monitor cells 3 and 4 ) regions of the tank. The mixing time for the near and far end are obtained when the following equalities are satisfied 


$$
\begin{array}{ll}
\frac{\max _{i, j}\left|\mathrm{C}-\mathrm{C}_{\mathrm{i}}\left(\mathrm{T}_{\mathrm{i}}-\tau_{\mathrm{i}}\right)\right|}{\mathrm{C}}=\delta & \mathrm{i}=1,2 \\
\frac{\max _{\mathrm{i}}\left|\mathrm{C}-\mathrm{C}_{\mathrm{i}}\left(\mathrm{T}_{\mathrm{i}}-\tau_{\mathrm{i}}\right)\right|}{\mathrm{C}}=\delta & \mathrm{i}=3,4
\end{array}
$$

In this criterion, the mixing time is given as the average of the near and far end mixing times.

It must be noted that these criteria are readily determinable for the present situation, with dissolved salt used as a passive scalar. With a known salt source term, the average concentration, $\overline{\mathrm{C}}$, is readily determinable. However, in situations where the average is not known $a$ priori, it is necessary to supplant these criteria with an additional requirement on the determination of $\bar{C}$. In mobilization of a sludge, for example, the fully mixed average concentration might be well known in a controlled laboratory experiment, whereas in the field, $\overline{\mathrm{C}}$ would probably not be a well known nor an easily determinable quantity. Obviously, estimates can be made based on known sludge depth and concentration, but additional caution is required to determine the extent of mixing in a full-scale field application.

\subsection{Time Dependent Concentration Curves}

The mixing criteria described in Section 6.1 require detailed knowledge of the concentration time history curves at several monitor locations. Several features in the concentration history curves were prevalent in all the saline water solution mixing simulations. Regardless of the jet velocity or whether one or two jets were used, the concentration time history curves that start from zero concentration can be classified as follows:

1. The concentration rapidly overshoots $\overline{\mathrm{C}}$ and then slowly decays toward $\overline{\mathrm{C}}$ in a nonmonotonic fashion (in some instances the concentration fluctuates like a strongly damped oscillation).

2. The concentration increases towards $\overline{\mathrm{C}}$, slightly overshooting or undershooting this value, and then slowly approaches $\bar{C}$ in a non-monotonic fashion.

Examples of these types of concentration time history curves are shown in Figures 6.1 and 6.2 , respectively. The concentration curves that were computed for the $1 / 6$ - and $2 / 3$-scale models are comprised of these two types of curves, with variations in the rates of growth and 
decay and different "periods" of the oscillating concentrations. These features of the concentration curves appeared regardless of the jet velocity, and were observed in both single- and double-jet mixing simulations of the $2 / 3$-scale tank.

Figures 6.3 and 6.4 show typical concentration time histories for the five cells used to define mixing times in the numerical simulations. It is because of this oscillatory behavior of the concentration at certain cells that a mixing criterion cannot be simply based on the time it takes for monitor cells to reach $\bar{C}$. For a specified percent deviation from $\bar{C}$, the mixing time must be traced backwards in time, regardless of the chosen criterion.

\subsection{Mixing Time Results}

Numerically computed mixing time results must be based on approximations to a global criterion. In this section, the mixing times based on the three criteria defined above are shown. It will be evident that when using truncated criteria, the mixing times are criterion dependent. For most cases, these discrepancies were less than $20 \%$; however, variations as high as $70 \%$ were also found.

The computational grid for the 1/6-scale tank simulations was set up according to the initial configuration of the tank used in the mixing experiments at ORNL. For the 2/3-scale tank, the grid was set up according to the 25,000 gallon model data provided by ORNL. Because of the location of the jet in the horizontal filled tanks, Cartesian coordinates proved to be more consistent than cylindrical coordinates. In early calculations, the cylindrical coordinate system was used, but the centerline induced non-physical mixing due to its being a no-flow location.

\subsubsection{Single Jet Simulations in a 1/6-Scale Tank}

For the 1/6-scale tank mixing computations, a momentum source simulated a jet with a discharge area equivalent to a 0.87 -in. diameter nozzle. The concentration monitor cells were located in pairs at each end of the tank. A computational cell containing a saline solution was placed in the middle of the tank adjacent to the upper wall at time zero, approximating a saline solution injection in the experiments. Several test simulations showed that as long as the fluid parcel containing the saline solution is near the top of the tank, its axial location will not appreciably affect the mixing time. In addition, if initially the volume occupied by the injected salty water is much smaller than the volume of the tank, the amount of saline solution will not influence the mixing time. In other words, it behaves like a truly passive scalar. 


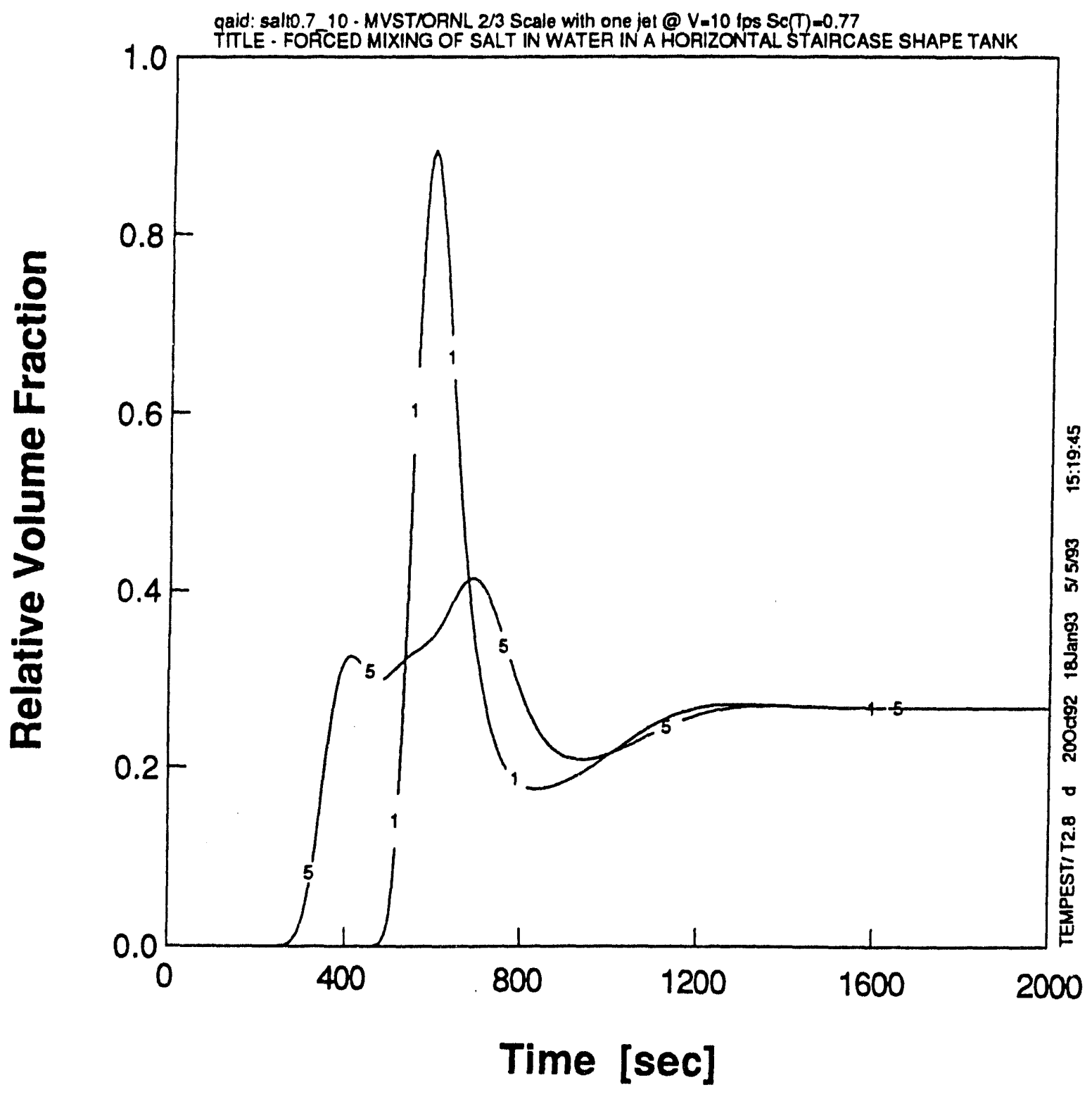

Figure 6.1. Examples of Concentration Curves Which Rapidly Overshoot the Average Concentration and Then Oscillate Toward It. This case corresponds to single jet mixing in water at $10 \mathrm{ft} / \mathrm{sec}$ in a $2 / 3$-scale tank. 


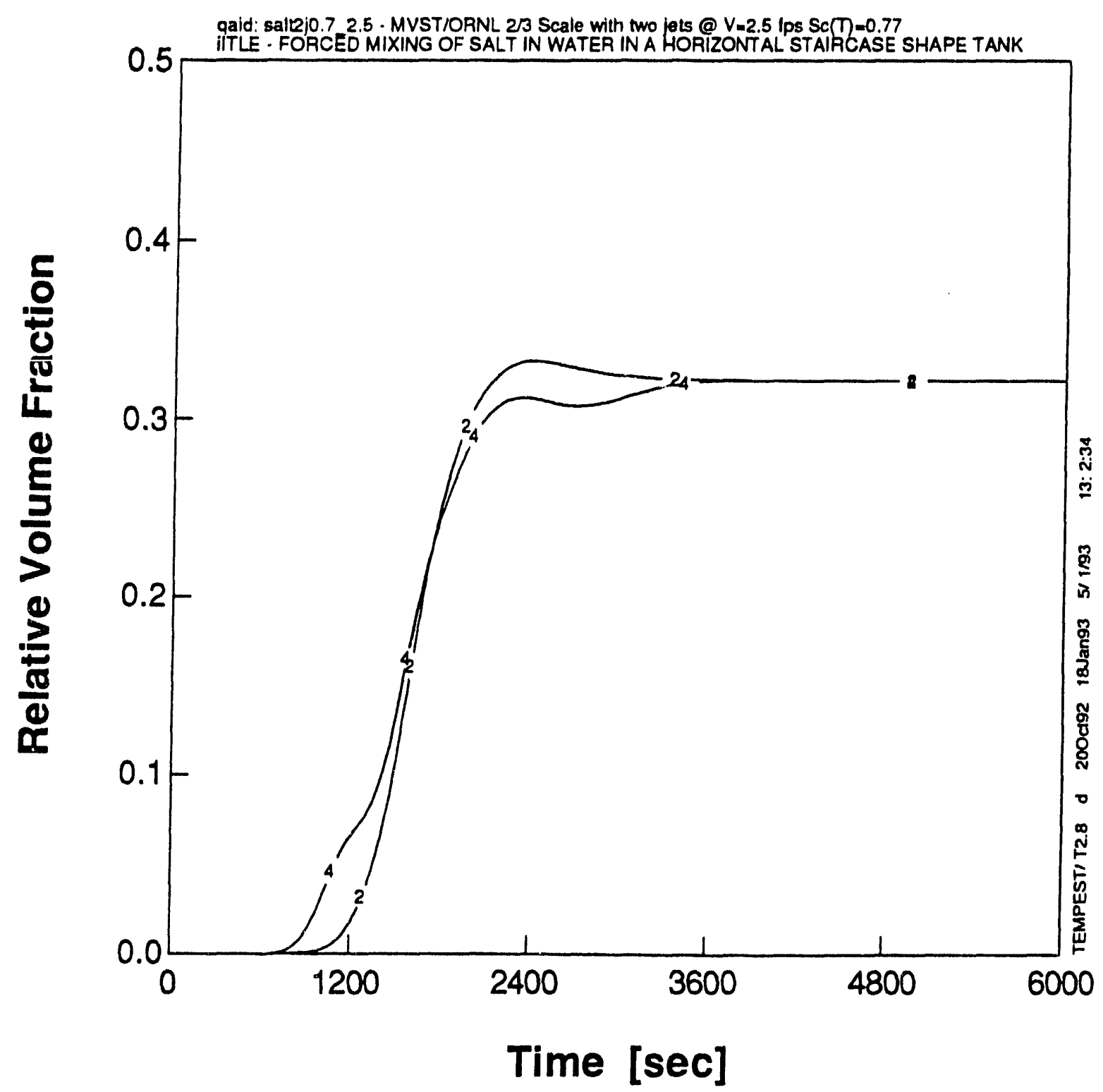

Figure 6.2. Examples of Concentration Curves Which Slightly Overshoot the Average Concentration. This case corresponds to double jet mixing in water at $2.5 \mathrm{ft} / \mathrm{sec}$ in a $2 / 3$-scale tank. 


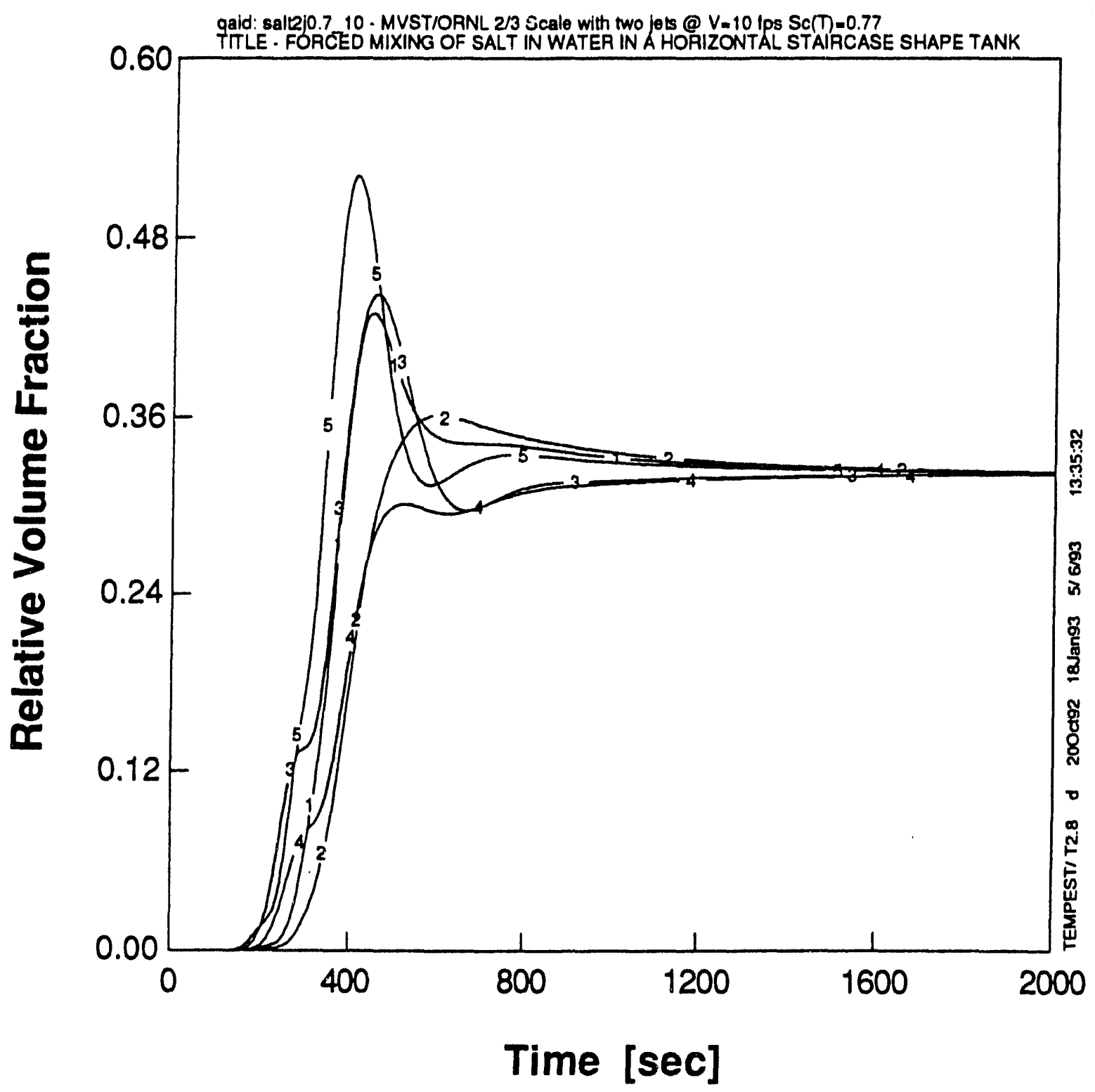

Figure 6.3. Typical Time Dependent Concentration Curves for Double Jet Mixing at High Velocity $(10 \mathrm{ft} / \mathrm{sec})$ in a $2 / 3$-Scale Tank 


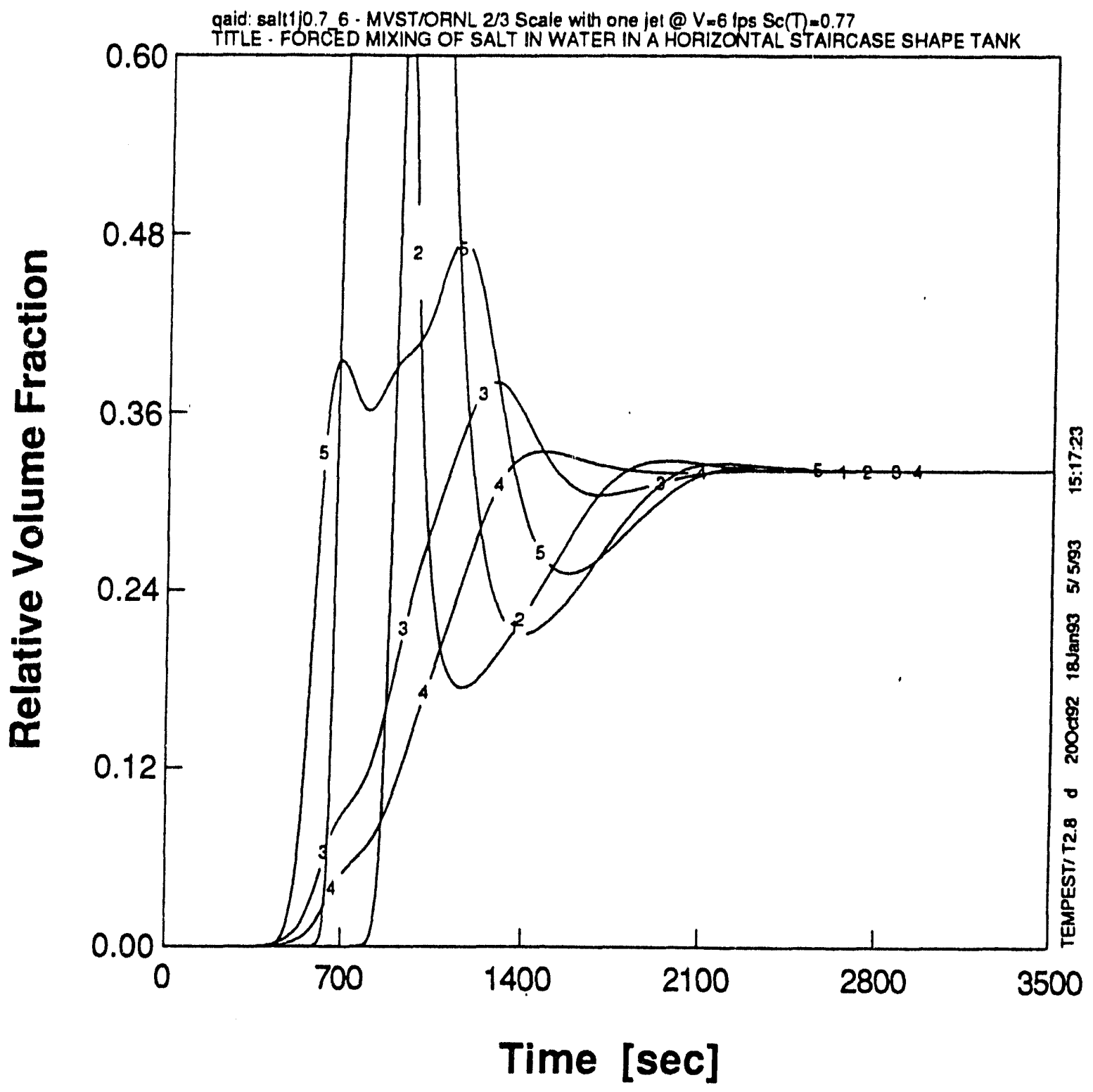

Figure 6.4. Typical Time Dependent Concentration Curves for Single Jet Mixing at Moderate Velocity $(6 \mathrm{ft} / \mathrm{sec})$ in a $2 / 3-$ Scale Tank

In Figure 6.5, the mixing times obtained computationally are compared with some early experimental results. Also included in the figure are data obtained from the experimental correlation of Okita and Oyama (Maruyama 1986). This correlation was developed for single jet mixing in a vertical tank orientation. The mixing times obtained from numerical 


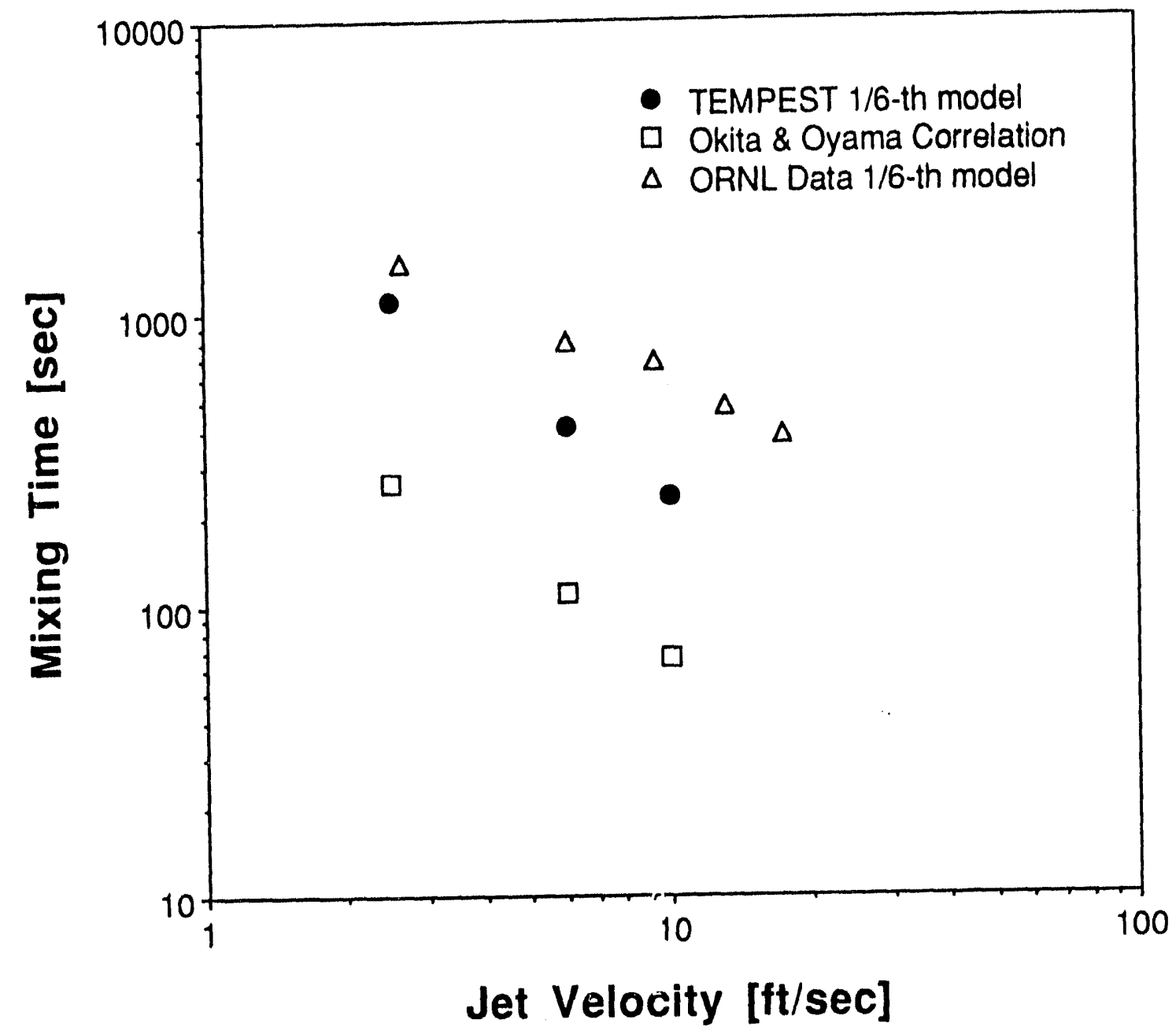

Figure 6.5. Comparison of Experimental (ORNL), Numerical (TEMPEST), and Correlated (Okita and Oyama) Mixing Time Values for a Fill ed 1/6-Scale Tank with Water

simulations were calculated using criterion 1 at $0.5 \%$. It is worth noting that there was a wide range of variation (up to a factor of two) in the early experimental data used to determine mixing times for the $1 / 6$-scale tank. ${ }^{(a)}$

In lieu of an experimental correlation formula for horizontal cylindrical tanks, the correlation of Okita and Oyama, which was developed for vertical cylindrical tanks, was used to provide a comparison estimate of mixing times. The correlation is given by

(a) Mr. J. Perona, ORNL, personal communication. 


$$
T=c \frac{d}{V_{j}}\left(\frac{D}{d}\right)^{1.5}\left(\frac{H}{d}\right)^{0.5}
$$

where $\mathbf{T}=$ Mixing time

$$
\begin{aligned}
c & =\text { Correlation constant }(=5.5) \\
\mathrm{d} & =\text { Nozzle diameter } \\
\mathrm{V}_{\mathrm{j}} & =\text { Jet velocity } \\
\mathrm{D} & =\text { Tank diameter } \\
\mathrm{H} & =\text { Tank Height (Length was used for the horizontal tanks) }
\end{aligned}
$$

This correlation is valid provided the jet Reynolds number $R e$ is in the range $5 \times 10^{3}<\operatorname{Re}<10^{5}$. For jet velocities between 2.5 and $10 \mathrm{ft} / \mathrm{sec}$ in the $1 / 6$-scale horizontal tanks, jet Re falls between $1.5 \times 10^{4}$ and $6 \times 10^{4}$, respectively. Okita and Oyama's correlation was obtained by measuring concentration differences at only two different locations within the tank. Thus, this correlation cannot be regarded as universal.

The mixing times obtained numerically and experimentally were based on the concentrations at five and four locations, respectively. Taking into account that the mixing time values in Figure 6.5 are based on different criteria, conclusions drawn from quantitative comparison must be interpreted with caution. The results are qualitatively similar, in that the mixing time decreases as jet velocity increases. If the constant $c$ in the above correlation were substituted by 21.4 , there would be agreement with the computational results within $8 \%$. If the constant $c$ were substituted by 40.4 , there would be agreement with the experimental results within $25 \%$.

\subsubsection{Single Jet Simulations in a 2/3-Scale Tank}

The computational grid for the 2/3-scale model was modified to the 2/3-scale tank dimensions. According to the tank diagrams provided, the scales are slightly different in both models. Table 6.1 illustrates the differences in the characteristic dimensions and their ratios.

The mixing times for a 5\% departure from $\overline{\mathrm{C}}$ are presented in Table 6.2 for the three criteria previously defined. The mixing times are strongly dependent on the mixing criterion. These results are plotted in Figure 6.6. For a $2.5 \mathrm{ft} / \mathrm{sec}$ jet velocity there is a $17 \%$ discrepancy between the time predicted by the first and the third criteria. The most conservative 
Table 6.1. Comparison of Dimension Ratios Between Scaled Tanks

\begin{tabular}{||l|c|c|c|c||}
\hline & $\begin{array}{c}\text { Nozzle } \\
\text { Diameter (in.) }\end{array}$ & $\begin{array}{c}\text { Tank Diameter } \\
\text { (in.) }\end{array}$ & $\begin{array}{c}\text { Tank Length } \\
\text { (in.) }\end{array}$ & $\begin{array}{c}\text { Bottom to } \\
\text { Nozzle } \\
\text { Distance (in.) }\end{array}$ \\
\hline \hline 1/6 Model & 0.87 & 23.56 & 122.75 & 1.25 \\
\hline 2/3 Model & 3.00 & 124.5 & 461.8 & 4.00 \\
\hline Ratio & 3.45 & 5.28 & 3.76 & 3.2 \\
\hline
\end{tabular}

Table 6.2. Single Jet Mixing Times in Water for a 2/3-Scale Tank

\begin{tabular}{|c|c|c|c|}
\hline $\begin{array}{c}\text { Jet Velocity } \\
(\mathrm{ft} / \mathrm{sec})\end{array}$ & $\begin{array}{c}\text { Mixing Time (sec) } \\
\text { Criterion 1 }\end{array}$ & $\begin{array}{c}\text { Mixing Time (sec) } \\
\text { Criterion 2 }\end{array}$ & $\begin{array}{c}\text { Mixing Time (sec) } \\
\text { Criterion 3 }\end{array}$ \\
\hline 2.5 & 4896.8 & 4784.4 & 4061.3 \\
\hline 6.0 & 2001.2 & 1949.2 & 1812.1 \\
\hline 10.0 & 1187.1 & 1164.2 & 1087.1 \\
\hline
\end{tabular}

estimates for the mixing times are given by the first criterion. The second and third criteria yield consistently lower mixing times.

Based on criterion 1, the effect of decreasing $\delta$, the percentage departure from $\overline{\mathrm{C}}$, is shown in Table 6.3. The values at $\delta=0.00$ (full mixing) were obtained by cubic extrapolation from the numerically computed mixing times at $\delta=0.05,0.04,0.02$, and 0.01 (see Figure 6.7).

\subsubsection{Double Jet Simulations in a 2/3-Scale Tank}

The computational grid used in this case was set up with the same dimensions as described in the previous section, except that two momentum sources issuing fluid in opposite directions were placed at 4 in. above the bottom of the tank. The double nozzle arrangement has a fluid intake in the center. This allows fluid to be replenished below the nozzles in a direction perpendicular to the nozzle axis. This set-up was chosen because it closely resembles a double jet system with suction near the bottom of the tank. However, computed mixing times with suction from the top were indistinguishable for the salt water system. This might not be the case with highly viscous or non-Newtonian fluids. 


\section{Mixing Times for the 2/3-th Scale Tank with One Jet (5\%)}

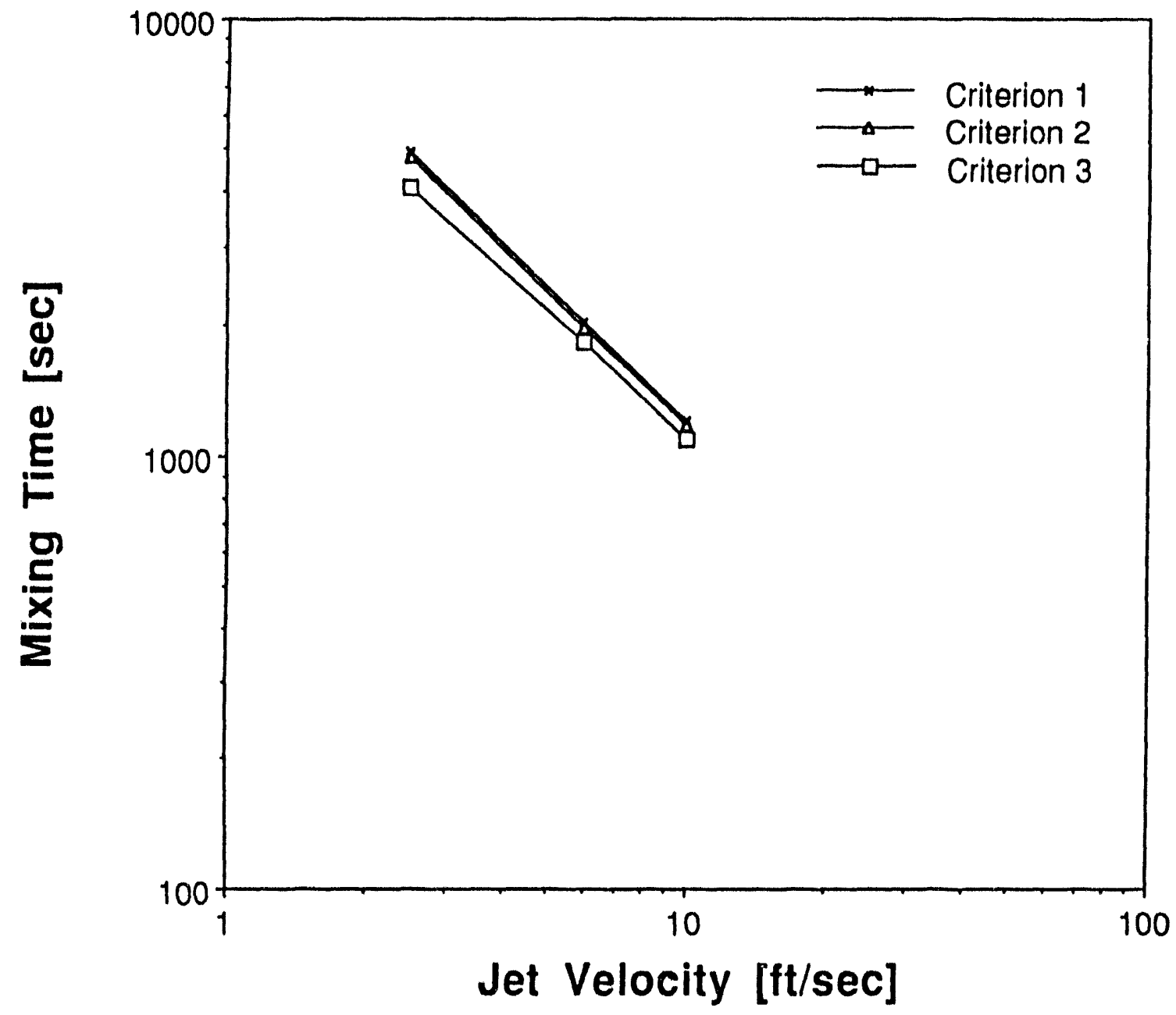

Figure 6.6. Single Jet Mixing Times as a Function of Jet Velocity for a 2/3-Scale Tank According to Three Different Mixing Criteria

Table 6.3. Effect of Percent Deviation from Average Concentration $\delta$ on Estimated Single Jet Mixing Times in a 2/3-Scale Tank

\begin{tabular}{|c|c|c|c|c|c|}
\hline $\mathrm{V}_{\mathrm{i}}(\mathrm{ft} / \mathrm{sec})$ & $\delta=0.05$ & $\delta=0.04$ & $\delta=0.02$ & $\delta=0.01$ & $\delta=0.00$ \\
\hline \hline 2.5 & 4896.8 & 5000.0 & 5161.3 & 5806.5 & 7219.5 \\
\hline 6.0 & 2001.2 & 2046.4 & 2114.1 & 2345.6 & 2848.2 \\
\hline 10.0 & 1187.1 & 1214.5 & 1262.9 & 1400.0 & 1689.8 \\
\hline
\end{tabular}




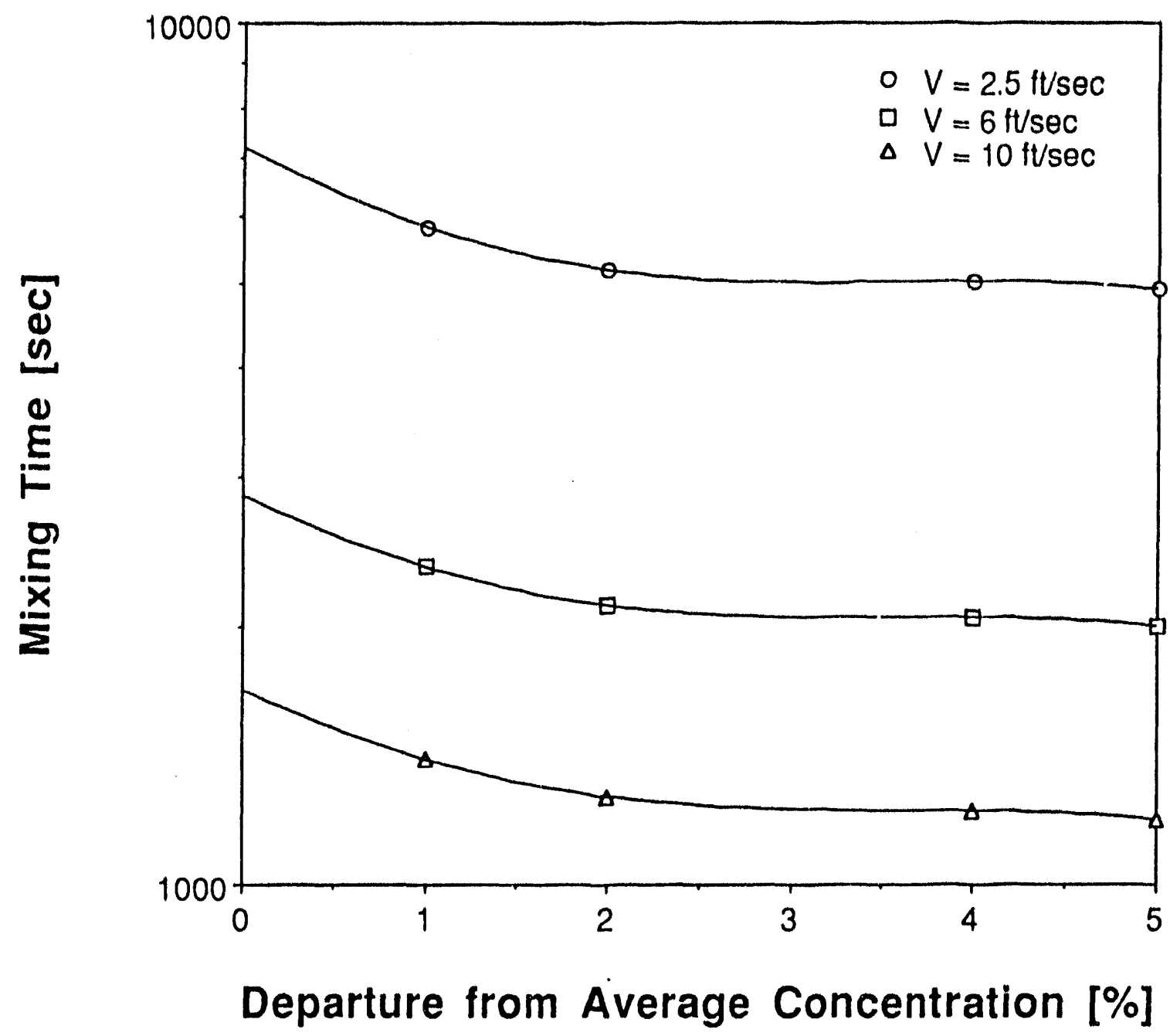

Figure 6.7. Single Jet Mixing Times as a Function of the Percent Departure from the Average Concentration for a 2/3-Scale Tank

Prior to computing the mixing times, it was verified that both jets have the same rate of spread close to the nozzle. Figure 6.8 shows the axial velocity component as a function of distance along the length of the tank. Note that at one quarter distance from the near end of the tank, where the double jet is located, the axial jet velocity is negative to the left and positive to the right; and for a distance of about $6 \mathrm{ft}$ both profiles are mirror images of each other.

For a 5\% departure from $\overline{\mathrm{C}}$, the mixing times for the three criteria previously defined are shown in Table 6.4. Figure 6.9 presents the mixing times as a function of jet velocity. Note that in the double jet case, the mixing times based on the third criterion are not consistently lower than those based on the second and first criteria. Because of practical limitations, one is 


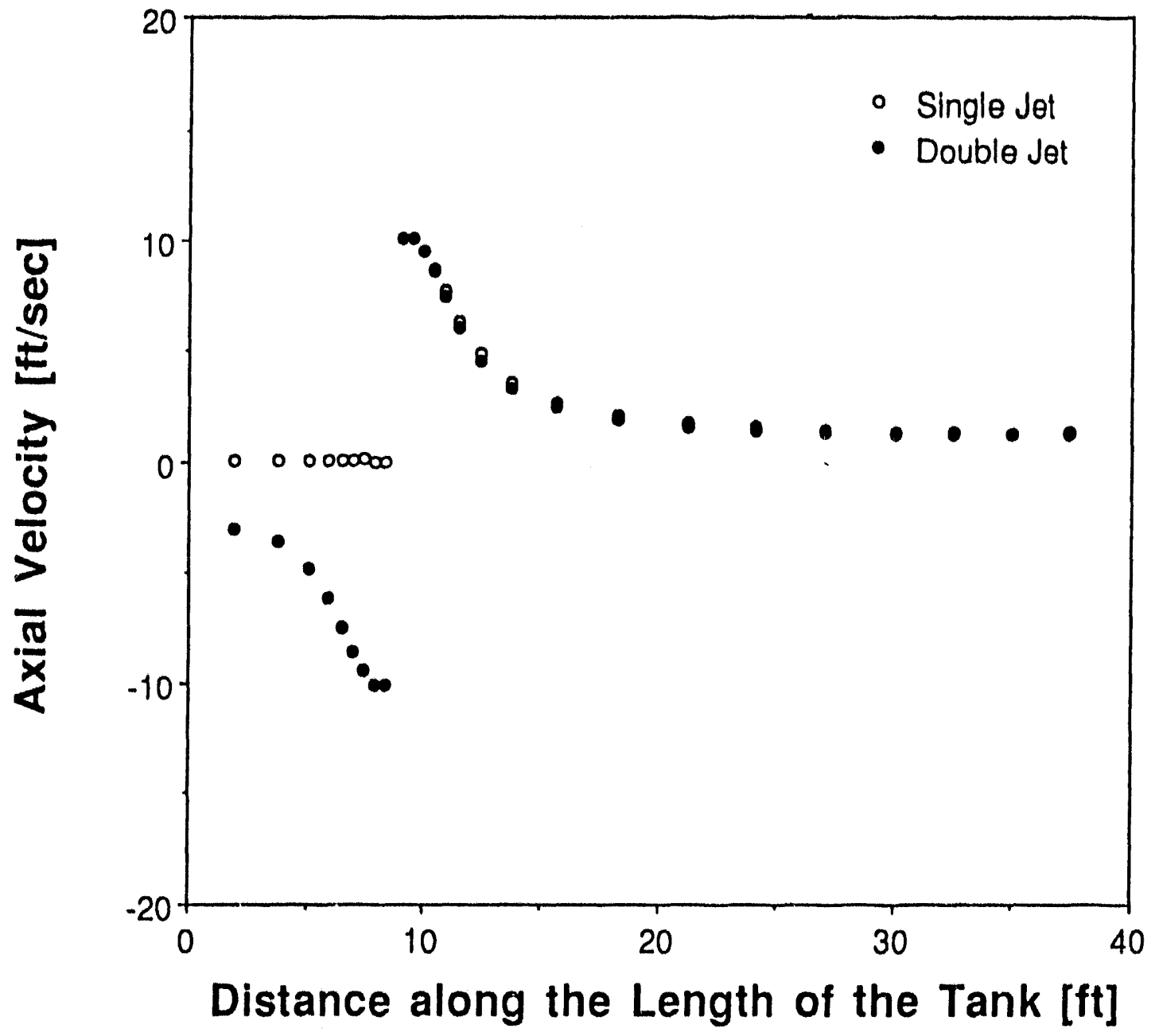

Figure 6.8. Comparison of the Axial Velocity Along the Jet Axis for Single and Double Jet Arrangements in a 2/3-Scale Tank

constrained to deal with mixing time criteria based on a relatively small number of sampling points. Consequently, a criterion selected for mixing time may yield counterintuitive results, as in the case of criterion 3 for the double jet. In lieu of a global mixing criterion, the use of criterion 1 is recommended because it yields the most consistent and conservative estimate of the mixing time, as compared to criteria 2 and 3.

Based on criterion 1, the effect of decreasing the departure from $\overline{\mathbf{C}}$ is shown in Table 6.5. The values at $\delta=0.00$ were obtained by cubic extrapolation from the numerically computed mixing times (see Figure 6.10). 


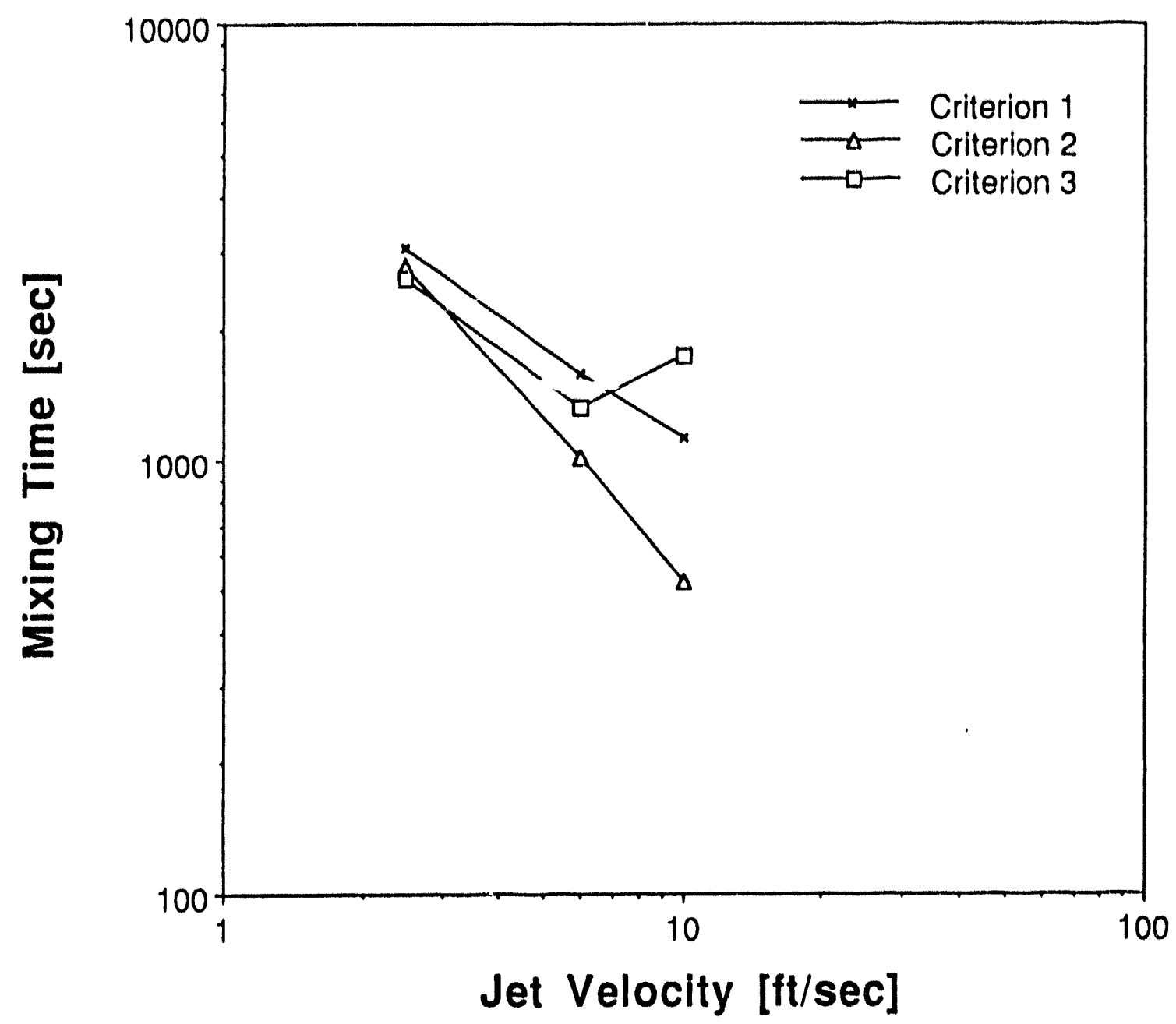

Figure 6.9. Double Jet Mixing Times as a Function of Jet Velocity for a 2/3-Scale Tank According to Three Different Mixing Criteria

Table 6.4. Double Jet Mixing Times in Water for a 2/3-Scale Tank

\begin{tabular}{||c|c|c|c||}
\hline $\begin{array}{c}\text { Jet Velocity } \\
(\mathrm{ft} / \mathrm{sec})\end{array}$ & $\begin{array}{c}\text { Mixing Time (sec) } \\
\text { Criterion 1 }\end{array}$ & $\begin{array}{c}\text { Mixing Time (sec) } \\
\text { Criterion 2 }\end{array}$ & $\begin{array}{c}\text { Mixing Time (sec) } \\
\text { Criterion 3 }\end{array}$ \\
\hline \hline 2.5 & 3058.1 & 2785.4 & 2600.8 \\
\hline 6.0 & 1564.5 & 1008.1 & 1308.1 \\
\hline 10.0 & 1112.9 & 522.0 & 1732.8 \\
\hline
\end{tabular}


Table 6.5. Effect of Percent Deviation ( $\delta$ ) from Average Concentration ( $\bar{C})$ on Estimated Double Jet Mixing Times in a 2/3-Scale Tank

\begin{tabular}{|c|c|c|c|c|c|}
\hline $\mathrm{V}_{\mathrm{i}}(\mathrm{ft} / \mathrm{sec})$ & $\delta=0.05$ & $\delta=0.04$ & $\delta=0.02$ & $\delta=0.01$ & $\delta=0.00$ \\
\hline 2.5 & 3058.1 & 3125.8 & 3275.8 & 3406.5 & 3606.6 \\
\hline 6.0 & 1564.5 & 1738.7 & 2193.5 & 2680.6 & 3478.5 \\
\hline 10.0 & 1112.9 & 1200.0 & 1500.0 & 1821.8 & 2330.7 \\
\hline
\end{tabular}

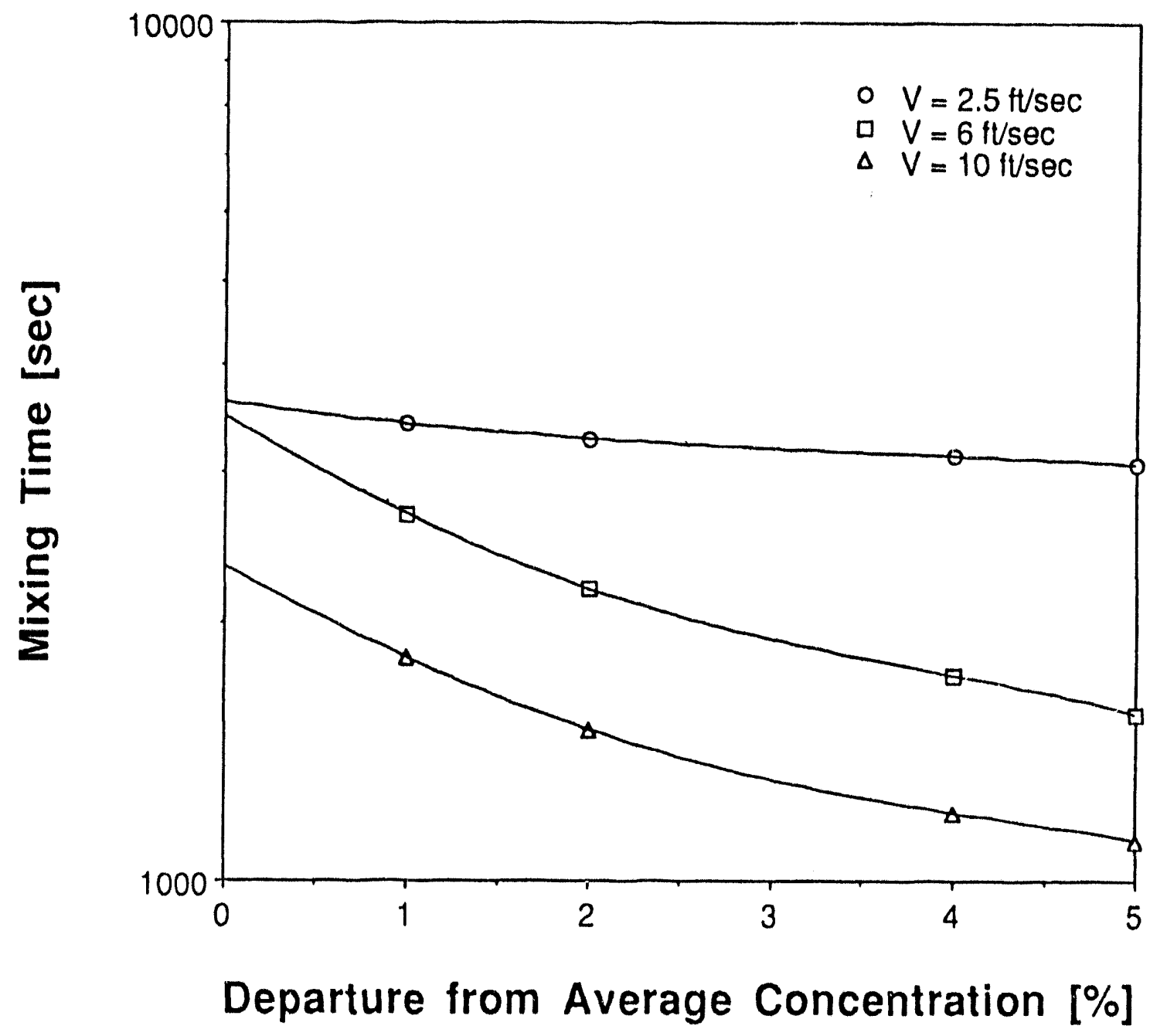

Figure 6.10. Double Jet Mixing Times as a Function of the Percent Departure from the Average Concentration for a 2/3-Scale Tank 


\subsection{Comparison Between Single and Double Jet Mixing}

Figure 6.11 shows the mixing times according to criterion 1 as a function of volumetric flow rate for several percentage departures from the average concentration. Note that once a $5 \%$ departure from the average has been reached, the time required to reach smaller (2\% and $1 \%)$ departures from the average is greater with two jets than with one jet. For a $5 \%$ departure from the average concentration, the effect of using two opposed jets at $2.5 \mathrm{ft} / \mathrm{sec}$ $(110 \mathrm{gpm})$ reduces the mixing time by $37.5 \%$ as compared to the single jet case $(55 \mathrm{gpm})$, whereas two jets at $10 \mathrm{ft} / \mathrm{sec}(440 \mathrm{gpm})$ reduce the mixing time by only $6.3 \%$. However, for a $1 \%$ departure form the average concentration, the mixing time is reduced by $41.3 \%$ with two jets at $2.5 \mathrm{ft} / \mathrm{sec}$ and increased by $23.2 \%$ with two jets at $10 \mathrm{ft} / \mathrm{sec}$. These results show that, in general, increasing the volumetric flow rate by using a double jet does not necessarily guarantee a reduction in the mixing time.

For the range of jet velocities studied, adding a diametrically opposed jet to the single jet arrangement decreases the rate at which the mixing times are diminished as the jet speed increases. The location of the jets at $1 / 4$ of the distance along the length of the tank is not the optimum location to minimize the time required for full mixing. In the present case, single jet mixing is more efficient than double jet mixing, because the power required to mix with two jets becomes impractical at high jet velocities $(10 \mathrm{ft} / \mathrm{sec})$. This result indicates that there is a location and orientation of the double jets such that the mixing time is minimized. It could be argued that such a location would be at the center of the tank. At this location, the left side of the tank is a mirror image of the right side, because the midplane that divides both sides becomes a no-flux boundary. This is equivalent to mixing each half of the tank with a single jet. 


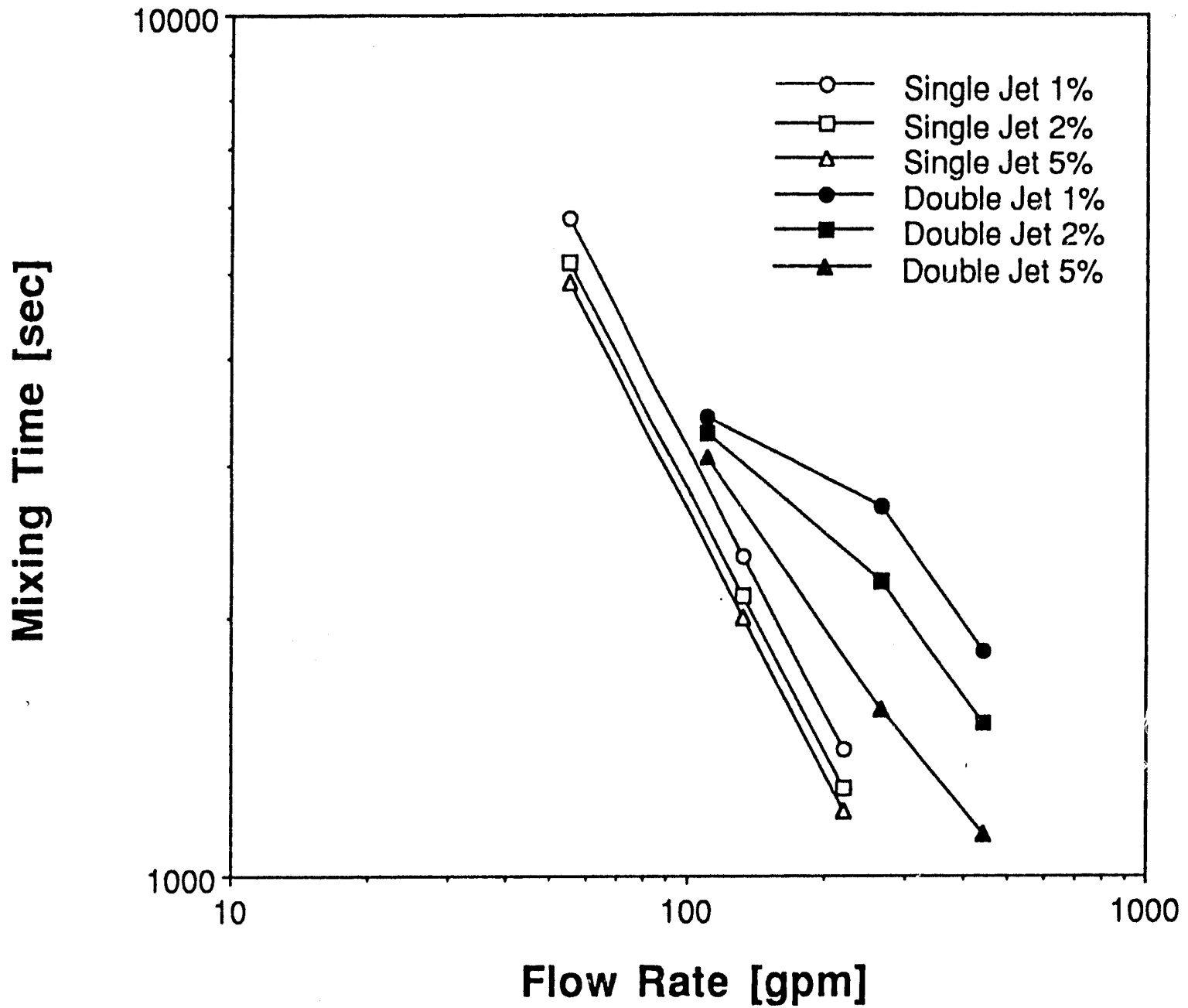

Figure 6.11. Comparison of Mixing Times as a Function of Flow Rate for Several Percentage Departures from the Average Concentration Based on Criterion 1 for Single and Double Jet Mixing in a 2/3-Scale Tank 


\subsection{Simulant Development}

An investigation ${ }^{(a)}$ was undertaken to develop a simulant to be used in 1/6- and 2/3-scaled mixing and mobilization experiments. The objective of this effort was to develop a waste simulant with rheological properties similar to ORNL W-28 sludge. Both solid sludge pure rheology and dilution factors were considered. Settling of particles was also considered so that the scaled experiments could be conducted in a sequential mode--whereby a settled layer is mobilized, the jets are then shut off, and material is allowed to resettle for the next set of test conditions.

To develop a simulant whose rheological behavior is exactly the same as the real sludge would require that the exact composition of the waste be known. All chemical constituents with the exception of the radioactive compounds could (theoretically) be mixed to obtain the ideal simulant. In most instances, however, this approach is not compatible with additional requirements demanded from a simulant; that it be inexpensive, readily available, nonhazardous, and readily disposable. With these constraints in mind, efforts to develop a waste simulant focused on two types of clay materials (bentonite and kaolin). Mixtures of these materials in water at various weight fractions (dilutions) were evaluated.

Initially, single component slurries of kaolin clay and of bentonite clay were prepared and characterized. Characterization consisted of slurry density and slurry rheology, with shear rates from 0 to $20 \mathrm{sec}^{-1}$. The kaolin slurries were initially thought to be unacceptable because the kaolin settles over periods of a few days, so that the rheology of the sludge layer would be continually changing over that time. It appeared instead that slurries of $\sim 8$ to $\sim 12 \mathrm{wt} \%$ bentonite would reproduce the desired behavior, but at significantly different dilutions than exhibited by the ORNL waste samples. Both the kaolin and bentonite slurries exhibit a much sharper decrease in effective viscosity with dilution than does the W-28 sludge.

A second series of slurries consisting of mixtures of kaolin and bentonite were prepared and tested. The rheological behavior of some of these mixtures was closer to the W-28 waste behavior, but the dilution effect was still greater than desired. Furthermore, the bentonite tends to stay suspended and inhibits the kaolin from settling as well. Because it was a requirement that a simulant settle in a reasonable period of time (a few days at most) to a predictable rheological behavior, a third series of kaolin slurries were prepared and analyzed. These consisted of $15 \mathrm{wt} \%$ slurries of kaolin in water or in $1 \mathrm{wt} \% \mathrm{NaCl}$ solution. The salt solution was

(a) Assistance of M. R. Elmore, Waste Technology Center, Pacific Northwest Laboratory, in this work is acknowledged. 
tested because an observation had been made in other work that the addition of salt to the liquid flocculates the fine particles and causes faster settling. The effect it would have on the rheology of the settler solids layer was not known, though.

In this third series of tests, the slurries were mixed in $500 \mathrm{ml}$ graduated cylinders, and settling behavior was noted over a period of -3 days. Both slurries (with and without salt) produced a sharply defined sludge/supernate interface as they settled. Both slurries settled to approximately the same percent solids sludge layers, and at approximately the same rate. Subsequently, a separate settling test was performed with a $30 \mathrm{wt} \%$ slurry of kaolin in water to determine the effect of starting concentration on settling rate and sludge density. After similar settling times, the sludge layer from the $30 \mathrm{wt} \%$ mixture had approximately the same $\mathrm{wt} \%$ and vol\% settled solids as either of the $15 \mathrm{wt} \%$ mixtures. Results of the settling tests are shown in Table 7.1. The apparent density of kaolin particles observed in tests ranged from 2.1 to $2.3 \mathrm{~g} / \mathrm{ml}$. Reported theoretical densities (calculated from crystal structure) ranged from 2.61 to 2.64 .

The rheology of the sludge layers was then characterized. First the supernate was carefully removed from the columns to minimize disturbance of the sludge layers. Then samples of the sludge layers were pipetted from the top and bottom zones of each sample. This was done to determine if compaction of the sludge varied with depth of the layer. The splits were later composited to evaluate rheology of the overall sludge layer as a function of dilution.

Figure 7.1 shows the plot of shear stress versus shear rate for the tup, bottom and composite sludge samples for the slurry without $\mathrm{NaCl}$. Figure 7.2 shows the same curves for the slurry with $1 \mathrm{wt} \% \mathrm{NaCl}$ in the supernate. Note that the compaction of the sludge layer does vary with depth for both sludges, although the uniformity of the up- and down-curves is better for the salt-containing slurry. This behavior has not been confirmed by replicate tests.

Figures 7.3 and 7.4 show the shear stress versus shear rate behaviors for the composited no-salt and $1 \%$ salt sludge samples: neat, diluted $1: 1$, and diluted $1: 3$ with their respective supernates. Neither sample exhibited the desired ideal behavior of the actual waste samples. Both simulants show greater reduction in shear stress with dilution than the W-28 rheograms.

The up curves do not exactly coincide with the down curves for either slurry, suggesting some slight time-dependent behavior. The W-28 sample shear stresses, measured at discrete shear rates (Ceo et al. 1990) are probably more like the down curves. The data curves were generated with the HAAKE viscometer. Curve fit coefficients determined by a power law model $\left(\tau=m \dot{\gamma}^{\mathrm{n}}\right)$ built into the rheogram data reduction software are shown in Table 7.2 separately for the up, down, and combined curves for each slurry. 
Table 7.1. Settled Solids Behavior for Kaolin Clay Slurries

\begin{tabular}{||l|l|}
\hline \multicolumn{1}{|c|}{ Slurry Type } & \multicolumn{1}{|c|}{ Settled Sludge Layer } \\
\hline \hline $15 \mathrm{wt} \%$ kaolin (no NaCl) & $34.1 \mathrm{wt} \%$ solids \\
& $19.4 \mathrm{vol} \%$ solids \\
$15 \mathrm{wt} \%$ kaolin $(1 \mathrm{wt} \% \mathrm{NaCl})$ & $36.0 \mathrm{wt} \%$ solids \\
& $19.7 \mathrm{vol} \%$ solids \\
$30 \mathrm{wt} \%$ kaolin (no NaCl) & $35.0 \mathrm{wt} \%$ solids \\
& $20.0 \mathrm{vol} \%$ solids \\
\hline
\end{tabular}

Higher salt concentrations in the supernate solutions may have some effect on sludge behavior. This is of potential concern because it could affect rheology with time if electric conductivity probes dependent upon salt concentration variations are used in experiments as a basis for measuring mixing time. The salt effect could be further investigated. Also, slurries using another type of solids may exibit behavior that is closer to the desired rheological properties, and additional studies could be conducted to identify such a material. In addition to investigating rheological effects, other concerns such as cost, availability, and/or disposal requirements would also have to be considered.

To determine the degree of success of the simulant in representing W-28 sludge, the power law constants (consistency factor, $\mathrm{n}$, and behavior index, $\mathrm{m}$ ) for the simulant mixtures given in Table 7.2 as a function of dilution can be compared to the dilution effects presented in Figures 4.1 and 4.2. Furthermore, an average effective viscosity of the power law representation over a strain rate from $1 \mathrm{sec}^{-1}$ to $20 \mathrm{sec}^{-1}$ can be computed according to

$$
\bar{\mu}\left(C_{\mathrm{r}}\right)=\frac{1}{\dot{\gamma}_{2}-\dot{\gamma}_{1}} \int_{\dot{\gamma}_{1}}^{\dot{\gamma}_{2}} \mu_{\text {eff }}(\dot{\gamma} ; \mathrm{m}, \mathrm{n}) \mathrm{d} \dot{\gamma}
$$

The results of Equation (7.1) are presented in Figure 7.5 for the W-28 sludge and the $15 \mathrm{wt} \%$ kaolin mixture with and without salt addition.

From these comparisons, it is concluded that the $15 \mathrm{wt} \%$ kaolin mixture is not an ideal simulant of W-28 sludge. The mixture does, however, exhibit many features which indicate it is a usable simulant. It exhibits a dilutable power law rheology; it exhibits settling into a 


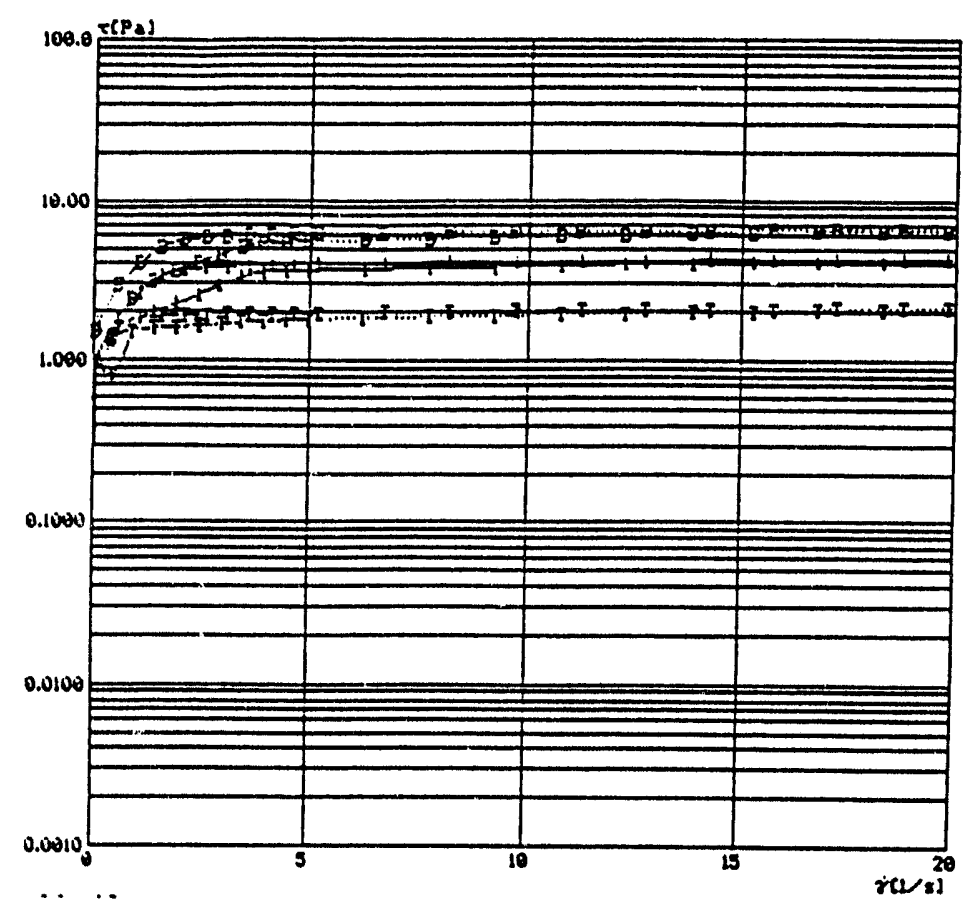

Figure 7.1. Shear Stress Versus Shear Rate Plot for $15 \mathrm{wt} \%$ Kaolin in Water Slurry. " $\mathrm{T}$ " = top section, "B" = bottom section, and middle curve is composite sample, respectively, from settled sludge layer

sludge layer which is congruent with planned experimental procedures, and it is readily obtainable. Therefore, using the developped kaolin simulant, the degree to which the differences in power law constants and average effective viscosity impact the objectives of scaled experiments can be established. However, if a primary objective of the scaled experiments is to develop data to scale up mixing and mobilization times, a much more extensive simulant development effort would be required. It would necessarily require that dimensionless scaling laws in non-Newtonian fluids be visited in some detail. This is not a trivial task. Alternatively, if a primary objective of the scaled experiments is to acquire quality mixing and mobilization data upon which computer simulations can be validated, then the kaolin simulant is probably adequate. The qualified computer modeling approach could then be used to make confident full-scale predictions. 


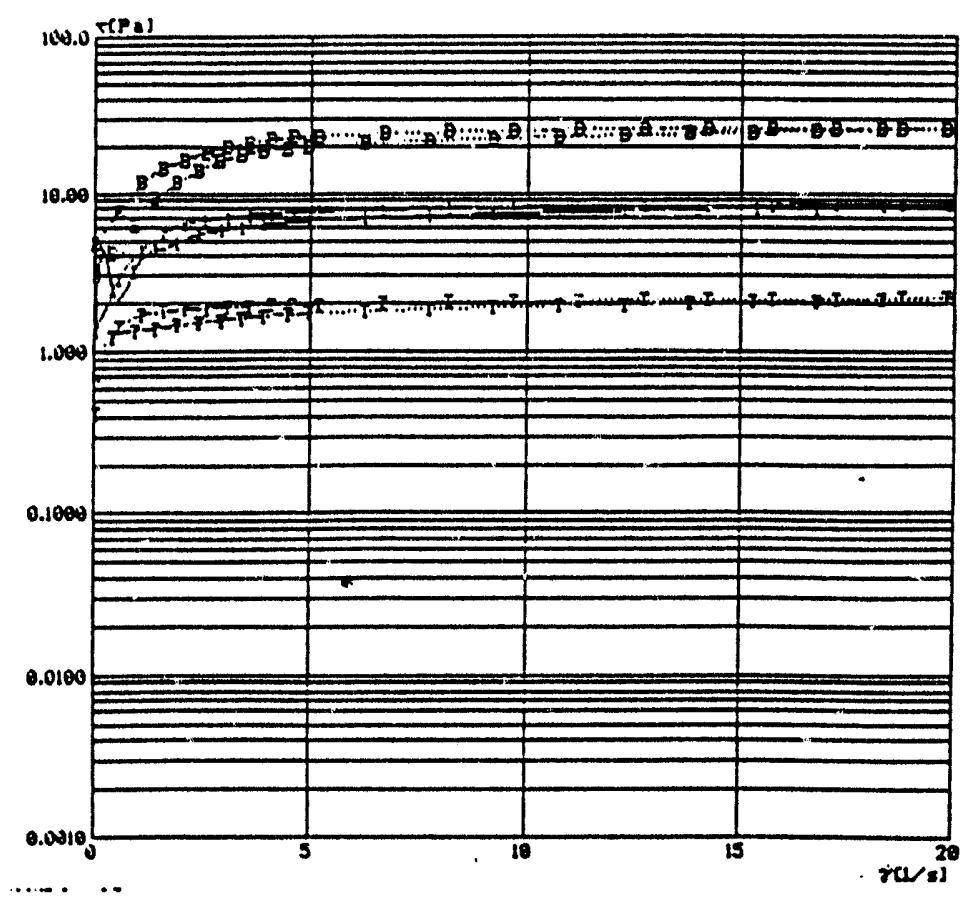

Figure 7.2. Shear Stress Versus Shear Rate Plot for 15 wt $\%$ Kaolin in 1 wt $\%$ $\mathrm{NaCl}$ Solution. "T" = top, "B" = bottom, and middle curve is composite sample, respectively, from settled sludge layer 


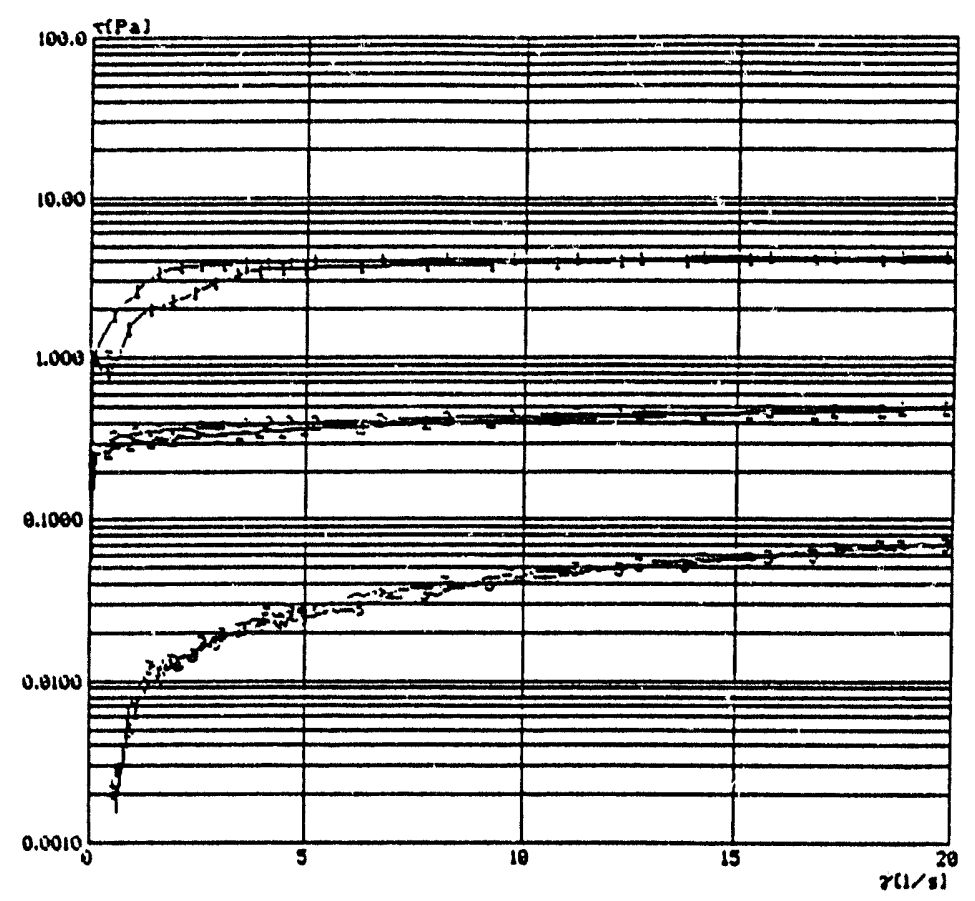

Figure 7.3. Shear Stress Versus Rate Plot for 15 wt \% Kaolin in Water Slurry. Top curve is undiluted sludge; middle curve is for $1: 1$ dilution by volume; bottom curve is for $1: 3$ dilution 
$L ' L$

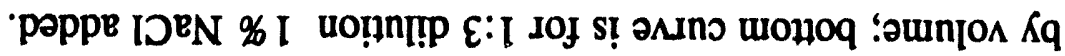

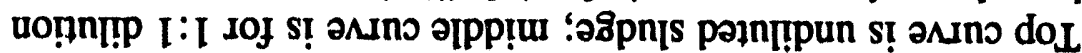

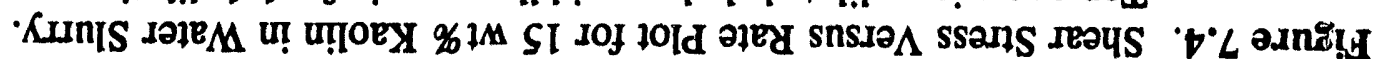

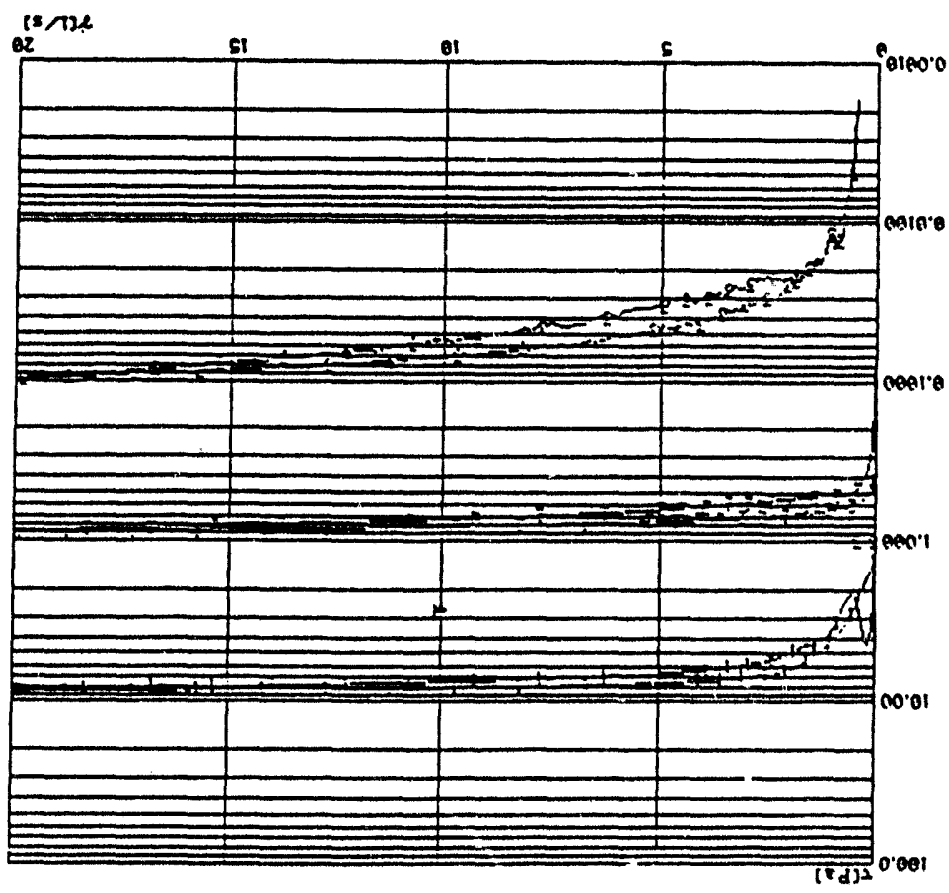


Table 7.2. Curve Fit Coefficients Using Power-Law Model $\left(\tau=m \dot{\gamma}^{\mathrm{n}}\right)$ for $15 \mathrm{wt} \%$ Kaolin Slurries in Water and in $1 \mathrm{wt} \% \mathrm{NaCl}$ Slurries. Neat values are for undiluted sludges. Dilutions ratios are based on volume.

\begin{tabular}{|c|c|c|c|c|}
\hline Slurry Type & Dilution Ratio & Up Curves & Down Curves & $\begin{array}{c}\text { Combined } \\
\text { Curves }\end{array}$ \\
\hline \multirow[t]{3}{*}{ No Salt Slurry } & Neat & $\begin{aligned} \mathrm{m} & =1.8050 \\
\mathrm{n} & =0.3383\end{aligned}$ & $\begin{aligned} \mathrm{m} & =2.6080 \\
\mathrm{n} & =0.2138\end{aligned}$ & $\begin{array}{l}\mathrm{m}=2.1670 \\
\mathrm{n}=0.2768\end{array}$ \\
\hline & $1: 1$ & $\begin{aligned} \mathrm{m} & =0.2902 \\
\mathrm{n} & =0.1569\end{aligned}$ & $\begin{array}{l}\mathrm{m}=0.3371 \\
\mathrm{n}=0.1145\end{array}$ & $\begin{aligned} \mathrm{m} & =0.3125 \\
\mathrm{n} & =0.1363\end{aligned}$ \\
\hline & $1: 3$ & $\begin{array}{l}\mathrm{m}=0.0069 \\
\mathrm{n}=0.7957\end{array}$ & $\begin{aligned} \mathrm{m} & =0.0059 \\
\mathrm{n} & =0.8 S 44\end{aligned}$ & $\begin{array}{c}\mathrm{m}=0.0063 \\
\mathrm{n}=0.8479\end{array}$ \\
\hline \multirow[t]{3}{*}{$1 \%$ Salt Slurry } & Neat & $\begin{array}{c}\mathrm{m}=4.0800 \\
\mathrm{n}=0.2648\end{array}$ & $\begin{aligned} \mathrm{m} & =4.3330 \\
\mathrm{n} & =0.2869\end{aligned}$ & $\begin{array}{c}\mathrm{m}=4.2060 \\
\mathrm{n}=0.2759\end{array}$ \\
\hline & $1: 1$ & $\begin{aligned} \mathrm{m} & =0.4659 \\
\mathrm{n} & =0.2071\end{aligned}$ & $\begin{aligned} \mathrm{m} & =0.6283 \\
\mathrm{n} & =0.1126\end{aligned}$ & $\begin{array}{l}\mathrm{m}=0.5395 \\
\mathrm{n}=0.1616\end{array}$ \\
\hline & $1: 3$ & $\begin{aligned} \mathrm{m} & =0.0110 \\
\mathrm{n} & =0.725\end{aligned}$ & $\begin{aligned} \mathrm{m} & =0.0121 \\
\mathrm{n} & =0.7663\end{aligned}$ & $\begin{array}{l}\mathrm{m}=0.0115 \\
\mathrm{n}=0.7466\end{array}$ \\
\hline
\end{tabular}




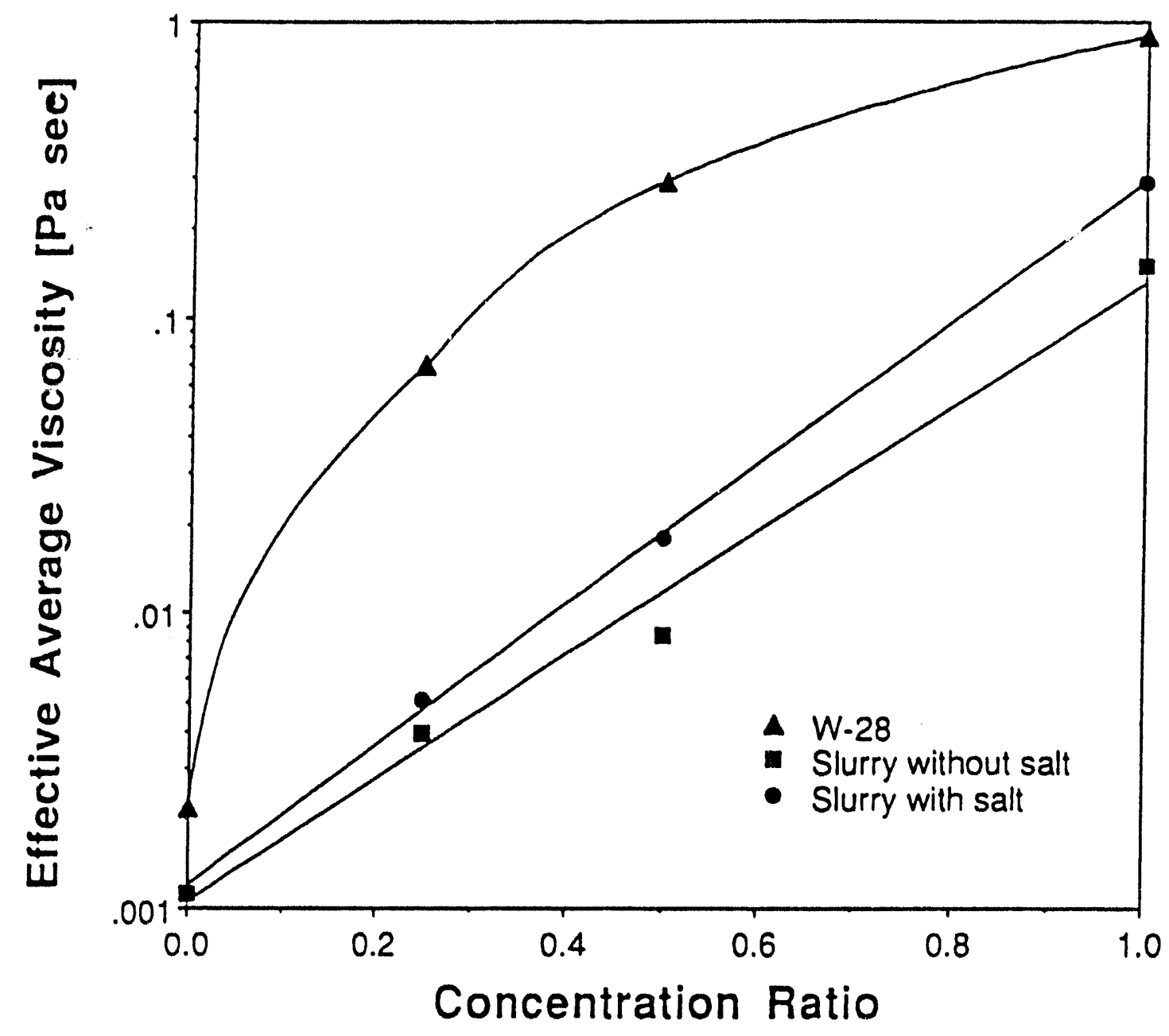

Figure 7.5. Comparison of the Effective Average Viscosity as a Function of Concentration Ratio Between the W-28

Waste Sludge and the Kaolin-Based Simulant 


\subsection{References}

Bamberger, J. A., L. M. Liljegren, and P. S. Lowery. 1991. A Methodology to Predict the Uniformity of Double-Shell Tank Waste Slurries Based on Mixing Pump Operation.

PNL-7664, Pacific Northwest Laboratory, Richland, Washington.

Bamberger, J. A., L. L. Eyler, and R. E. Dodge. 1993. Mathematical Modeling of Mixer Pump Performance for Agitation of Radioactive Slurries in One-Million Gallon Underground Storage Tanks at Hanford. PNL-8295, Pacific Northwest Laboratory, Richland, Washington.

Bird, R. B., R. C. Armstrong, and O. Hassager. 1987. Dynamics of Polymeric Liquids:

Fluid Mechanics. John Wiley and Sons, New York.

Ceo, R. N., M. B. Sears, and J. T. Shor. 1990. Physical Characterization of Radioactive Waste Tank Sludges. ORNL/TM-11653, Martin Marietta Energy Systems, Oak Ridge National Laboratory, Oak Ridge, Tennessee.

Darby, R. 1986. "Hydrodynamics of Slurries and Suspensions." In Encyclopedia of Fluid Mechanics, Vol. 5. Gulf Publishing Company, Houston, Texas.

Eyler, L. L., and T. E. Michener. 1992. "Computer Modeling of Forced Mixing in Waste Storage Tanks." In Proceedings of the Third International High-Level Radioactive Waste Management Conference, Las Vegas, Nevada, Vol. 1, pp. 636-642.

Fossett, H., and L. E. Prosser. 1949. "The Application of Free Jets to the Mixing of Fluids in Bulk." In Proceedings of the I. Mechanical Engineers, Vol. 160, p. 128.

Govier, G. W., and K. Aziz. 1987. The Flow of Complex Mixtures in Pipes.

Robert E. Krieger Publishing Company, Malabar, Florida.

Maruyama, T. 1986. "Jet Mixing of Fluids in Vessels." In Encyclopedia of Fluid Mechanics, Vol. 2. Gulf Publishing Company, Houston, Texas.

Perry, R. H., and C. H. Chilton. 1973. Chemical Engineers Handbook, 5th Edition. McGraw-Hill, New York.

Rajaratnam, N. 1976. Developments in Water Science: Turbulent Jets." Elsevier Scientific Publishing Co., Amsterdam.

Rice, P. 1986. "Batchwise Jet Mixing in Tanks." In Encyclopedia of Fluid Mechanics, Vol. 2. Gulf Publishing Company, Houston, Texas. 
Shor, J. T., and R. L. Cummins. 1991. MVST Sludge Mobilization Development. ORNL/TM-11709, Oak Ridge National Laboratory, Oak Ridge, Tennessee.

Tatterson, G. B. 1991. Fluid Mixing and Gas Dispersion in Agitated Tanks. McGraw-Hill, Inc., New York.

Trent, D. S., and L. L. Eyler. 1992. TEMPEST: A Computer Program for ThreeDimensional Time-Dependent Computational Fluid Dynamics. Battelle Memorial Institute, Columbus, Ohio.

Youngblood, E. L., J. B. Berry, and J. R. De Vore. 1991. "Predicting Transport Behavior for Radioactive Sludges at Oak Ridge National Laboratory." Presented at the AICHE Summer Meeting, August 18-21, 1991, Pittsburgh, Pennsylvania. Martin Marietta Energy Systems, Onk Ridge National Laboratory, Oak Ridge, Tennessee. 


\section{Distribution}

No. of

Copies

OFFSITE

2 DOE Office of Scientific and Technical Information

35 Oak Ridge National Laboratory Building 3503, MS-6316 P.O. Box 2008 Oak Ridge, TN 37831

Attn: C. H. Brown, Jr.

C. H. Byers

A. D. Clay

A. G. Croff

T. L. Donaldson

D. L. Daugherty

J. S. Davidson

J. R. Forgy

R. K. Genung

J. K. Gilpin

O. W. Hale

J. H. Hooyman

T. D. Hylton

L. L. Jacobs

L. L. Kaiser

C. M. Kendrick

T. E. Kent

E. Krispin

R. C. Mason

C. P. McGinnis

L. E. McNeese

J. W. Moore

T. J. Newsom

J. J. Perona

R. 5. Pudelek

S. M. Robinson

T. F. Scanlan

C. B. Scott

J. L. Snyder
No. of

Copies

R. C. Stewart

J. R. Trabalka

M. W. Tull

D. W. Turner

M. L. Whitehead

E. L. Youngblood

ONSITE

DOE Richland Operations Office

R. F. Christensen

R3-72

4 Westinghouse Hanford Company

T. M. Burke

S4-58

W. L. Knecht

HO-34

T. B. McCall

HO-33

E. D. Waters

S4-58

27 Pacific Northwest Laboratory

W. J. Apley

P7-46

J. A. Bamberger

K7-15

J. M. Bates

K7-15

I. G. Choi

K7-15

M. R. Elmore

P8-30

L. L. Eyler (5)

K7-15

B. M. Johnson

K1-78

N. J. Lombardo

K7-02

E. W. Pearson

K7-15

M. R. Powell

P7-19

F. M. Ryan

K7-70

P. A. Scott

P7-19

G. Terrones (5)

K7-15

Publishing Coordination K1-06

Technical Report Files (5) 

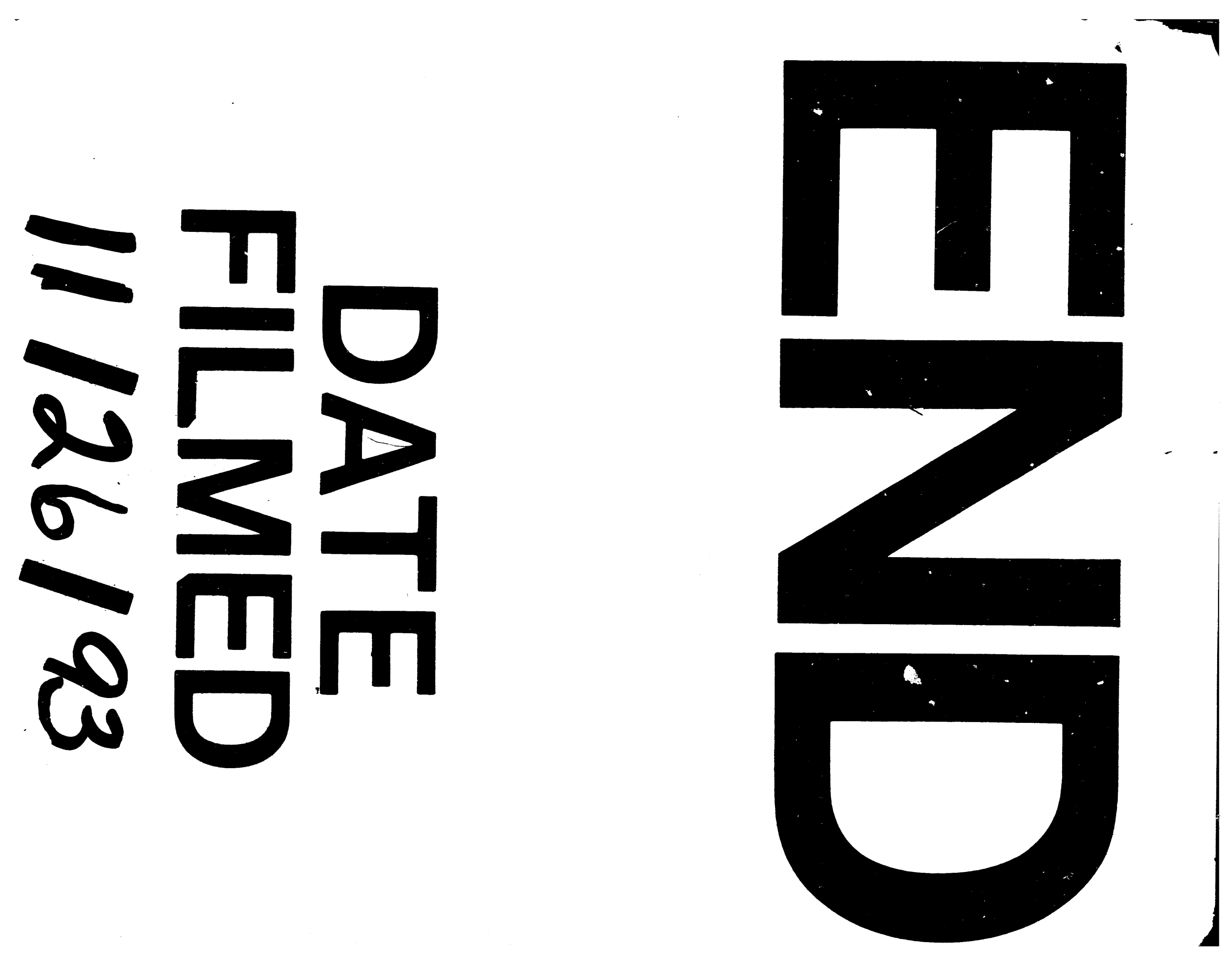
